\title{
A Microcantilever Platform for Measuring Internal Friction in Thin Films Using Thermoelastic Damping for Calibration
}

\author{
Guruprasad Sosale \\ Doctor of Philosophy \\ Mechanical Engineering \\ McGill University \\ Montreal, Quebec
}

A thesis submitted in partial fulfillment of the requirements for the degree of Doctor of Philosophy

(C) Guruprasad Sosale, July 2011 


\begin{abstract}
Measuring structural damping due to internal friction in deposited thin films can provide useful guidelines for the design of high-Q micro/nanomechanical resonators utilized used for sensing, communications and vibration energy harvesting. Such measurements can also generate valuable insights into the effects of size and confinement on the mechanisms of damping in these films. Typically, these measurements are carried out by depositing a thin film on a relatively thick cantilevered substrate and measuring the change in damping in the substrate/film composite. However, the measured damping is due to losses from several sources including support losses, clamping losses, thermoelastic damping (TED) and internal friction losses in the substrate/film composite. Accurately identifying the magnitude of internal friction in the film has been a long-standing difficulty in this field. This thesis presents the design and development of a silicon microcantilever platform to resolve this difficulty. At the core of the platform is the ability to reduce the damping in single crystal silicon microcantilevers to low levels of damping approaching the fundamental limits of dissipation established by thermoelastic damping (TED). This demonstrates that all other sources of damping (viscous losses, support losses and internal friction losses) are sufficiently low. These cantilevers can then be coated with the thin film of interest and the damping in the substrate/film composite measured. The measured damping is comprised of the thermoelastic damping in the substrate/film composite and the internal friction in the film. The former can be calculated using accurate models and the initial damping can be substracted from the measured damping in the composite to obtain an accurate estimate of the internal friction in the film.
\end{abstract}

In this dissertation, single crystal silicon cantilevers ranging from $8-130 \mu \mathrm{m}$ in thickness, and fundamental frequencies ranging from $80 \mathrm{~Hz}-40 \mathrm{kHz}$ were fabricated. Using the measurement platform, these beams are demonstrated to have low damping (logarithmic decrement $\approx 6 \times 10^{-6}$ ) with values approaching the TED limit. Subsets of these beams are then used to carry out the first calibrated 
measurements on the effects of thickness and frequency on internal friction in thin films of aluminum, gold and silver at room temperature. The films ranged in thickness from $60 \mathrm{~nm}$ to $450 \mathrm{~nm}$ and were studied over frequencies from $100 \mathrm{~Hz}$ to $1.5 \mathrm{kHz}$. The results of this study provide two valuable guidelines for the design of low dissipation (high Q) layered composite resonators: gold leads to a smaller increase in damping than either aluminum or silver, and damping in the composite resonators can be decreased by reducing the film thickness. As a third design guideline, a strategy to control and minimize the damping by selective patterning of thin films on the resonator is described. In this work, a simple model is developed to predict the damping in partially metalized cantilevered beams and experimentally validated using the platform. The silicon microcantilever platform, and the results obtained using this platform establish a foundation for studying internal friction in thin films. 


\section{Sommaire}

Mesurer l'amortissement structurel causé par friction interne dans les films minces déposés permet d'établir des recommandations pour le design de résonateurs micro/nanomécaniques ayant un facteur de qualité élevé, ainsi qu'apporter une meilleure compréhension de l'effet de la taille et du confinement sur le mécanisme d'amortissement de ces films minces. Ce type de résonateurs est utilisé dans les microsystèmes électromécaniques de détection, de communication et de récupération d'énergie vibratoire. Ces mesures d'amortissement sont réalisées en déposant un film mince sur un substrat ayant une forme de poutre en porte-à-faux et d'épaisseur relativement élevée, et en mesurant le changement d'amortissement de l'ensemble substrat/film. Cependant, l'amortissement global a des origines multiples, notamment des pertes provenant du support, du système de fixation, de l'amortissement thermoélastique, et de la friction interne dans l'ensemble substrat/film. Identifier avec précision l'amplitude de la friction interne dans le film est une difficulté de connue de longue date dans le milieu de la recherche scientifique. Cette thèse présente le design et le développement d'une plateforme en silicium ayant une micro-poutre en porte-à-faux afin de résoudre ce problème. L'intérêt principal de cette plateforme est que l'amortissement dans les micro-poutres en porte-à-faux faites en silicium monocristallin est réduit à un faible niveau approchant les limites théoriques de la dissipation établies par les lois de l'amortissement thermoélastique. Cela démontre que les autres sources d'amortissement (amortissement visqueux, pertes liées au support ou à la friction interne du substrat) sont suffisamment faibles. Par la suite, ces poutres peuvent être recouvertes par le film mince étudié et l'amortissement de l'ensemble substrat/film est mesuré. Cette mesure provient à la fois de l'amortissement thermoélastique dans l'ensemble substrat/film et de la friction interne dans le film mince. Cette dernière peut être calculée grâce à ces modèles précis, et l'amortissement initial du substrat peut être soustrait de l'amortissement mesuré pour l'ensemble substrat/film afin d'en déduire une estimation précise de la friction interne dans le film mince uniquement. 
Les poutres en porte-à-faux en silicium monocristallin fabriquées dans le cadre de cette thèse avaient une épaisseur comprise entre 8 et $130 \mu \mathrm{m}$, et une fréquence fondamentale comprise entre $80 \mathrm{~Hz}$ et $40 \mathrm{kHz}$. En utilisant la plateforme de mesure évoquée précédemment, le faible amortissement (décrément logarithmique $\approx 6 \times 10^{-6}$ ) approchant la limite thermoélastique théorique de ces poutres est démontré. Ce type de poutres est ensuite utilisé pour mener les premières mesures calibrées portant sur l'effet de l'épaisseur et de la fréquence sur la friction interne dans les films minces d'aluminium, d'or, et d'argent à température ambiante. Les films minces avaient une épaisseur comprise entre 60 $\mathrm{nm}$ et $450 \mathrm{~nm}$, et furent testés à des fréquences comprises entre $100 \mathrm{~Hz}$ et 1,5 $\mathrm{kHz}$. Les résultats de cette étude établissent deux recommandations pour le design de résonateurs à couches minces à faible dissipation (haut facteur de qualité) : l'or induit une augmentation de l'amortissement qui est plus faible que celle induite par l'aluminium ou l'argent, et l'amortissement des résonateurs peut être diminué en réduisant l'épaisseur de la couche mince. Une troisième recommandation est donnée sous la forme d'une stratégie pour contrôler et minimiser l'amortissement en déposant le film mince sur le résonateur selon un certain motif au lieu d'une couche continue. Dans ce travail de recherche, un modèle simple est développé pour prédire l'amortissement des poutres en porte-à-faux partiellement recouvertes de métal. Ce modèle est ensuite validé expérimentalement grâce à la plateforme en silicium. Cette plateforme ainsi que les résultats obtenus en l'utilisant établissent les fondements de l'étude de la friction interne dans les films minces. 


\section{Acknowledgments}

First and foremost, I would like to thank my supervisor, Prof Srikar Vengallatore. Over the past four years, he has been my mentor, guide and friend. He challenged me to do the best I could, provided me with sincere and careful feedback, and gave me focussed direction. By giving me the room to explore and develop as a researcher, he has made my academic experience one of the most rewarding and growing four years of my life. Srikar, I cannot thank you enough.

The path to this $\mathrm{PhD}$ topic took a number of twists and turns. In early 2008, Srikar provided me with the opportunity to transition from a biomedical related topic to study damping in microscale devices - a dramatic transition to say the least. It was here that I came under the co-supervision of Prof Luc Fréchette. Luc had the patience to allow me to develop my knowledge base, helped me focus my research efforts and encouraged my independent thought throughout the course of my $\mathrm{PhD}$. What I am most grateful for is that he taught me how to ask the right questions to evaluate whether what I was chasing after was important and pertinent. Luc, thank you for your guidance and help over the past few years.

Much of the last two years of my PhD were spent in the McGill Nanotools microfabrication facility. Were it not for the tremendous technical guidance, and the advice given to me by Don Berry and Dr Matthieu Nannini from this facility, all the measurements and developments in this thesis would have taken a lot longer to achieve. They truly are the backbone of many students' success. I would like to thank Prof Luc Mongeau and Prof Damiano Pasini for the use of the laser Dopler vibrometer without which many of the measurements would not have been possible. I would like to acknowledge NSERC for the funding that allowed me to pursue this degree without having to worry about having a stable income.

I want to thank my colleagues Sairam Prabhakar and Kaushik Das for their help over the course of this thesis. Both of them saw me at different points over this period of time. I had the chance to work with Sairam at the inception of this 
system. He had to bear with my incessant questions on thermoelastic damping and listen to my frustrations and joys as we inched closer to the thermoelastic damping target. It was his work that laid the foundation for my thesis. I had the pleasure to work with Kaushik at the tail end of this dissertation. The numerous discussions we had and his help with the verification of my code were of invaluable assistance to me.

My experience at McGill could not have been complete without all the people in FDA012 - the office I called home for many years. Meysam Rahmat, my good friend and colleague shared a large part of my academic and social experience. I thank him for all the wonderful discussions and the insights- both personal and professional- that he shared with me. A heartfelt thanks to Lacey Lemaire for helping proofread many sections of this thesis, and Julien Caubergh for his help with the translation of the abstract.

To Heide, thank you so much for your understanding, your help with proofreading, your insightful questions that drove me to reinforce my explanations and helping me mentally through the past three gruelling months. You gave me strength when I most needed it. Finally, I thank my parents. Their support, encouragement, passion for education and love is the only reason I have been able to make this journey. 


\section{Table of Contents}

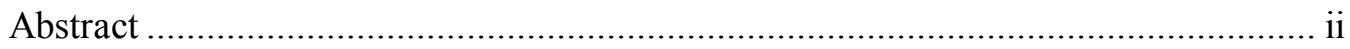

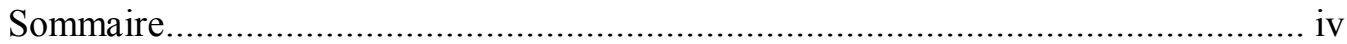

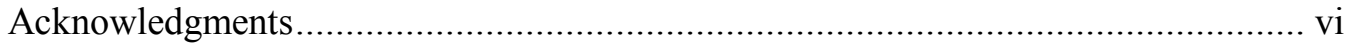

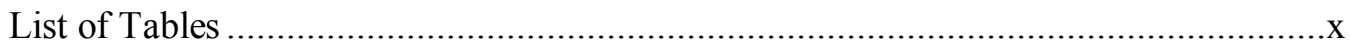

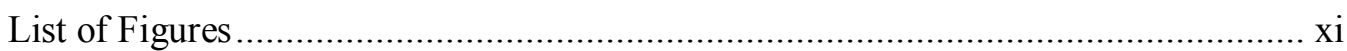

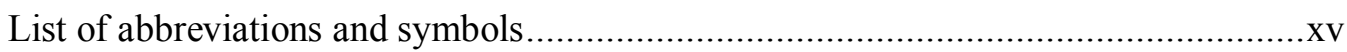

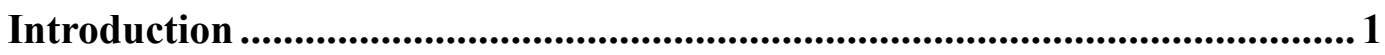

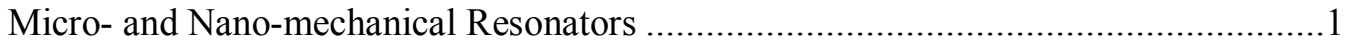

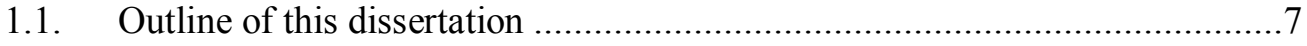

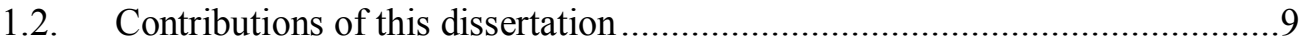

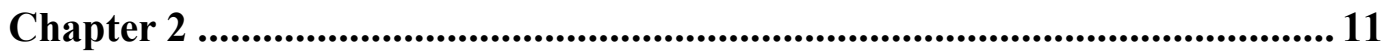

An Approach to Measuring Damping in Thin Functional Films Using Thermoelastic

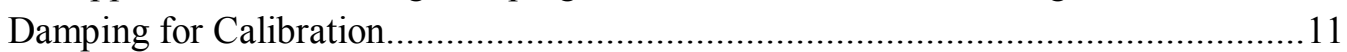

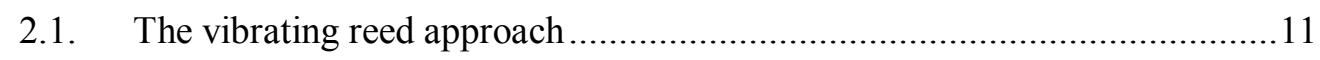

2.2. Theory: calibration using thermoelastic damping.................................. 14

2.3. Design and development of the measurement setup .................................21

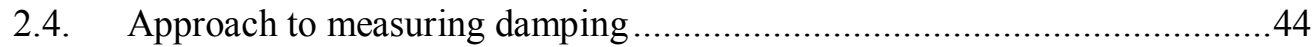

2.5. Measurement of damping in micromachined single-crystal silicon resonators

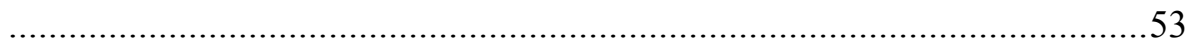

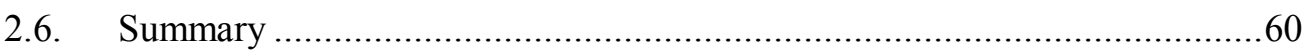

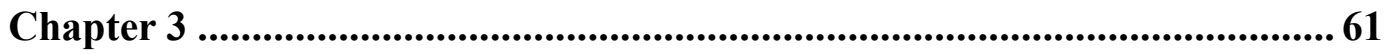

A Study of Internal friction in Thin films of Aluminum, Silver and Gold at Room

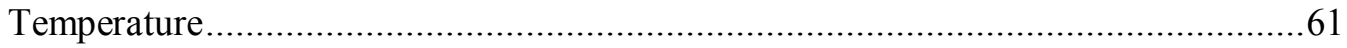

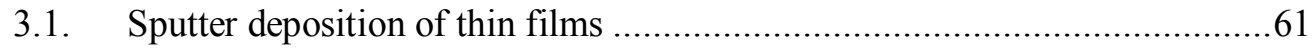

3.2. Characterization of stress and surface topography in sputtered thin films of

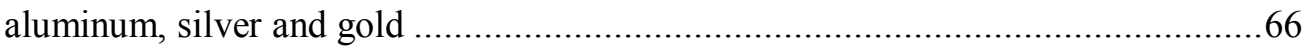

3.3. Measurement of damping in aluminum, silver and gold films ................... 74

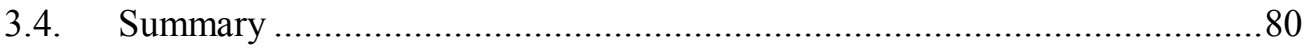


Measurement and Analysis of Internal Friction in Thin Films of Aluminum over Three

Decades of Frequency

4.1. Resolution of measurement for internal friction in thin films using the vibrating reed approach

4.2. Design, fabrication and measurement of high frequency single-crystal silicon

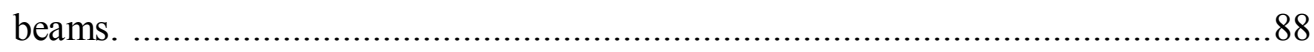

4.3. Measurement of damping in thin films of aluminum................................92

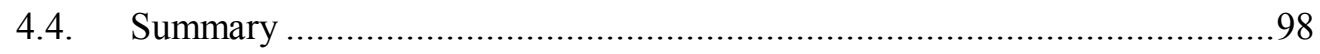

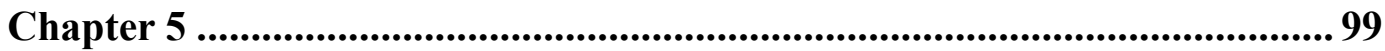

Controlling Damping and $Q$ Factors of Silicon Microcantilevers by Selective

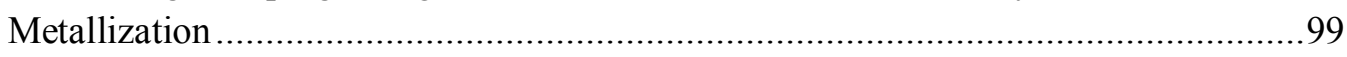

5.1. Model for the effects of patterning on damping .................................. 100

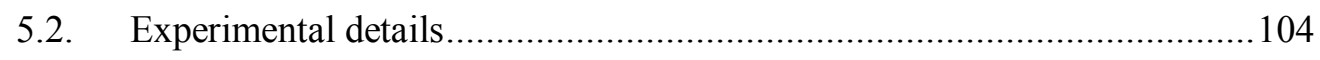

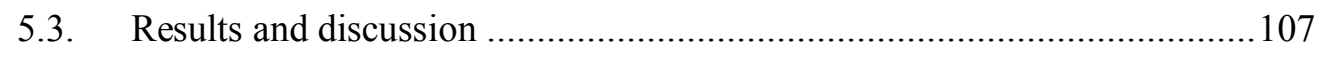

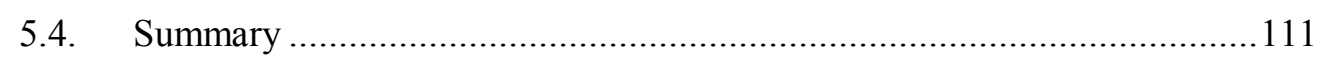

\section{Chapter 6 .................................................................................................................. 113}

The Design of a Microcantilever System to Measure Damping as a Function of

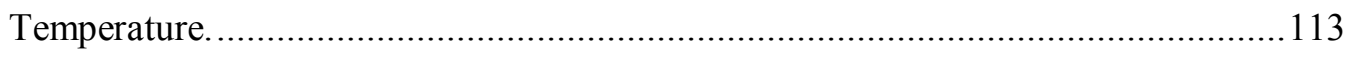

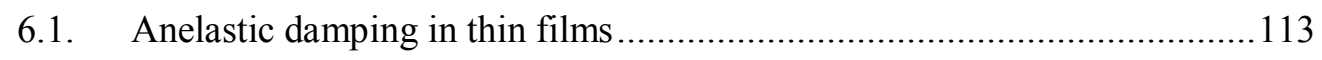

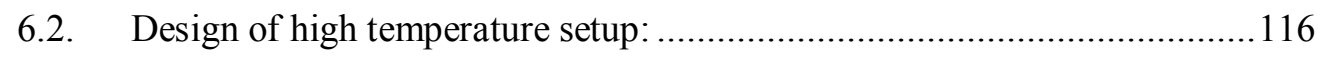

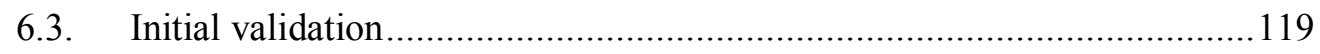

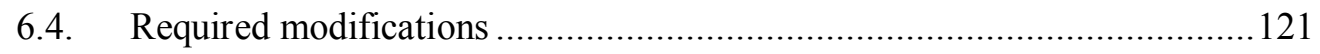

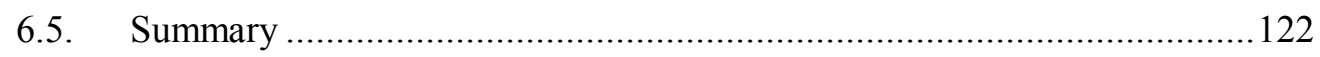

Conclusions and Future work ............................................................ 123

7.1. Guidelines for the design of layered resonators................................... 124

7.2. Studies on internal friction in thin films as a function of frequency........... 125

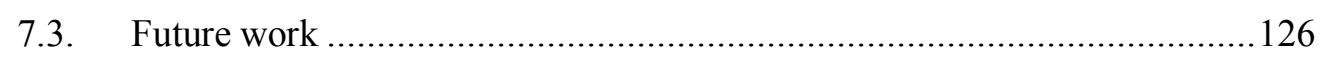

Appendix ................................................................................................. 129

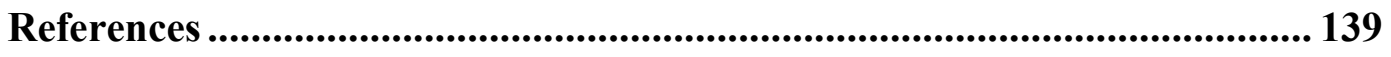




\section{List of Tables}

Table 1.1: Design and operating parameters of micro/nanomechanical resonators [Ven08].......

Table 2.1: Various reed configurations used for internal friction studies. 26

Table 2.2: Process flow for fabricating of low frequency single crystal silicon beams with a step at the base. 28

Table 2.3: Specification of Nano-OP65 piezoelectric stage used for base excitation. .....32

Table 2.4: Capabilities of the fiberoptic displacement sensor and the laser Doppler Vibrometer.

Table 2.5: Repeatability measurements of eight beams remounted at least three times with the same clamp setup.

Table 3.1: Deposition parameters and measured residual stress for the sputtered thin films

Table 4.1: Predicted minimum resolution in four $100 \mu \mathrm{m}$ thick silicon cantilevers used to measure internal friction in a $100 \mathrm{~nm}$ thick $\mathrm{Al}$ film as a function of frequency. . .86

Table 4.2: Process flow for fabrication of high frequency ( $>1500 \mathrm{~Hz}$ ) single crystal silicon beams starting with an silicon-on-insulator (SOI) wafer.

Table 4.3: Measurements of damping in the 20 single-crystal silicon cantilevers used in this study.

Table 5.2: Results of damping in the first mode of flexural vibration. 108

Table 6.1: Results of damping measurements with the piezoelectric shaker and electrostatic device described in section 6.2.

Table A1.1: Comparison of measured and predicted decay frequencies for each mode of the lightly damped silicon beam

Table B.1: Measurements of pristine single crystal silicon beams shown in Figure

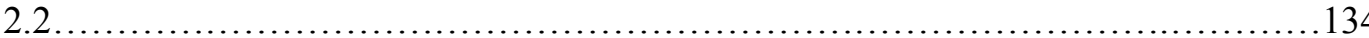

Table B.2: Detailed data for measurements of damping in thin films of $\mathrm{Al}, \mathrm{Ag}$ and $\mathrm{Au}$ presented in section 3 .... 


\section{List of Figures}

Figure 1.1: Scanning electron micrographs of common MEMS resonator and anchor

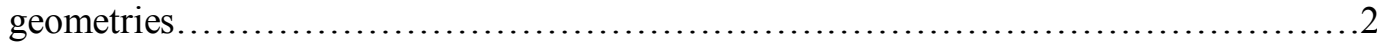

Figure 2.1: Schematic of a microfabricated silicon cantilevered beam attached to a large supporting structure.

Figure 2.2: Graph of thermoelastic damping as a function of the normalized frequency for silicon beams calculated using Equation (2.6).

Figure 2.3: Schematic of components of the complete damping measurement system. ..21

Figure 2.4: Schematic of a microfabricated silicon cantilevered beam attached to a base with a finite thickness but infinite width [Pho04].

Figure 2.5: Schematic illustration of the strain field at the root of an oscillating cantilever

Figure 2.6: Micromachined silicon cantilevers produced by the process outlined in table

Figure 2.7: a) Exploded view of the subcomponents in the clamp assembly b) photograph of clamp attached to the shaker which in turn is attached to a large stainless steel block that serves as the frame for the system.

Figure 2.8: The gain of shaker as a function of frequency

Figure 2.9: a) Design schematic showing shaker, clamp and beam when loaded into chamber b) Photograph of the high-vacuum assembly designed for measurements. 35

Figure 2.10: Power spectrum of vertical acceleration signal from an accelerometer mounted on the pneumatic vibration isolation table.

Figure 2.11: Photograph of shaker, clamp and beam setup with fiber optic probe positioned to measure the displacement of the beam.

Figure 2.12: Photograph of the test apparatus used for measuring the dynamics of microcantilevers.

Figure 2.13: a) power spectrum of vertical acceleration of the LDV b) magnitude of the frequency response of the acceleration of the LDV to the table.

Figure 2.14: Representative signals of the displacement of the shaker base measured with the built in CDS and the LDV at $20 \mathrm{~Hz}$.

Figure 2.15: Plot of displacement of the shaker as a function of applied voltage as measured by the LDV

Figure 2.16: Plot of the free decay signal of a silicon cantilever and its envelope as calculated from as described in Equation (2.31). 
Figure 2.17: Measurement of beam dynamics using laser Doppler vibrometry. The graph shows the tip velocity as a function of time during harmonic excitation and free decay. .51

Figure 2.18: Power spectral density of: a) velocity of the tip of silicon cantilever measured with LDV and b) displacement of piezo shaker base measured with the capacitative sensor during a free decay.

Figure 2.19: Representative pressure dependence of damping (logarithmic decrement) in single-crystal silicon beams as a function of pressure in the vacuum chamber.

Figure 2.20: Logarithmic decrement measured in 70 different single-crystal silicon microcantilevers operating in the first mode.

Figure 2.21: Residual damping ( $\left.\delta_{s}-\delta_{s, T E D}\right)$ as a function of a) beam thickness, b) aspect ratio.

Figure 2.22: Residual damping ( $\delta_{s}-\delta_{s, T E D}$ ) as a function of a) beam length, b) frequency.

Figure 3.1: Schematic of the effect of deposition pressure on the stress in sputter deposited thin films [Win92].

Figure 3.2: Illustration of the effect of internal stress of a thin film on a thick substrate [Mad02].

Figure 3.3: Effect of argon pressure on the film stress in $\mathrm{Al}$ and $\mathrm{Al} / \mathrm{Cr}$ films

Figure 3.4: Plot of intrinsic stress in aluminum, silver and gold films under the conditions in Table 3.1 .

Figure 3.5: $1.5 \times 1.5 \mu \mathrm{m}^{2}$ atomic force microscopy (AFM) images of $60 \mathrm{~nm}$ thick aluminum film ....

Figure 3.6: $1.5 \times 1.5 \mu \mathrm{m}^{2}$ Atomic force microscopy (AFM) images of $430 \mathrm{~nm}$ thick aluminum film

Figure 3.7: Representative $1.5 \times 1.5 \mu \mathrm{m}^{2}$ AFM images of silver films of $420 \mathrm{~nm}$ thick film.

Figure 3.8: Representative $1.5 \times 1.5 \mu \mathrm{m}^{2}$ AFM images of gold films of $420 \mathrm{~nm}$ thick film.

Figure 3.9: Photograph of custom machined molybdenum clamp and wafer for sputter deposition of films onto the beams.

Figure 3.10: Effect of thin adhesion layers (15 nm Ti, $15 \mathrm{~nm} \mathrm{Cr}$ ) on damping of singlecrystal silicon microcantilevers.

Figure 3.11: Increase in damping due to metallization with thin films of aluminum $(\boldsymbol{\Delta})$, silver $(\boldsymbol{\square})$ and gold $(+)$. .76 
Figure 3.12: Effect of thickness on internal friction in thin films of aluminum $(\boldsymbol{\Delta})$, silver

(匹) and gold (+).

Figure 3.13: Frequency dependence of internal friction in $110 \mathrm{~nm}$ thick films of aluminum $(\boldsymbol{\Lambda})$, silver $(\mathbf{\square})$ and gold $(+)$.

Figure 4.1: Schematic of two single crystal silicon wafers that are bonded to form a silicon on insulator (SOI) wafer.

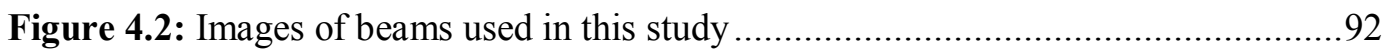

Figure 4.3: Frequency dependence of the damping in the beams used in this study.. .....94

Figure 4.4: Frequency dependence of internal friction in $60 \mathrm{~nm}$ thick films of aluminum.

Figure 5.1: Schematic illustration of a microcantilever beam of length $L$ with a thin film coated over a portion of the top surface.

Figure 5.2: Volumetric strain energy distribution in the first mode of flexural vibration of a cantilevered Euler Bernoulli beam. 104

Figure 5.3: Photographs of representative single-crystal silicon beams patterned selectively with $100 \mathrm{~nm}$ thick films of aluminum.

Figure 5.4: Graph of the measured increase in damping due to metallization as a function of pattern geometry for microcantilevers vibrating in the first mode. The curve represents the predicted fractional increase in dissipation with patterning along the length of the cantilever starting from the tip and was calculated from Equation (5.6).

Figure 5.5: Graph of the measured increase in damping due to metallization as a function of pattern geometry for microcantilevers vibrating in the first mode. The curve represents the predicted fractional increase in dissipation with patterning along the length of the cantilever starting from the base and was calculated from Equation (5.6).

Figure 6.1: Schematic illustration of anelastic behavior with time [Meh07].

Figure 6.2: Standard anelastic solid model where $J_{R}$ and $J_{U}$, are the relaxed and unrelaxed compliance respectively [Now72].

Figure 6.3: Design schematic of high temperature damping measurement setup using electrostatic actuation [Image from Sha11].

Figure 6.4: Plot of temperature as a function of time comparing the actual temperature of the clamp to the input set-point temperature [Sha11].

Figure A1.1: Decay of lightly damped beam excited in the first mode then in the third mode. a) Immediately after cutting the excitation b) after 13 seconds showing weaker $3^{\text {rd }}$ mode signal. 
Figure A1.2: a) Power spectral density of silicon cantilever decaying from excitation close to the $3^{\text {rd }}$ resonant mode. b) the envelope of the decay signals for each isolated mode from calculated using equation 2.31.

Figure A1.3: Logarithmic decrement as a function of normalized frequency for a $40 \mu \mathrm{m}$ thick cantilever measured in the first four resonant modes. 


\section{List of abbreviations and symbols}

\begin{tabular}{ll} 
Abbreviations & \\
AFM & Atomic force microscopy \\
Al & Aluminum \\
Au & Silver \\
BOE & Gold \\
BG & Buffered oxide etchant \\
CDS & Background damping \\
Cr & Capacitative displacement sensor \\
DRIE & Chromium \\
HF & Deep reactive ion etching process \\
High-Q & Hydrogen fluoride \\
IF & High quality factor \\
KV & Internal friction \\
LDV & Kelvin-Voigt model for material damping \\
RIE & Laser Doppler Vibrometer \\
SEM & Reactive ion etching \\
SOI & Scanning electron microscopy \\
TED & Silicon on insulator \\
Ti & Thermoelastic damping \\
TMAH & Titanium \\
\hline & Tetra methyl ammonium hydroxide \\
\hline &
\end{tabular}

\section{Latin Symbols}

$A$

C

$C_{d}$

$c$
Amplitude of oscillation

Specific heat per unit volume

Strain rate damping constant for material exhibiting KV behavior

The speed of propagation of shear wave in a solid 


\begin{tabular}{|c|c|}
\hline E & Young's Modulus \\
\hline$F$ & Force \\
\hline$f c c$ & Face-centered cubic metal \\
\hline$h$ & Thickness \\
\hline$H$ & Hilbert transform operation \\
\hline$I$ & Area moment of inertia \\
\hline$J$ & Compliance \\
\hline$k$ & Thermal conductivity \\
\hline$k_{b}$ & Boltzmann constant \\
\hline$k_{c}$ & Equivalent stiffness of cantilever \\
\hline$L$ & Length of microcantilever beam \\
\hline$M$ & Modulus \\
\hline$Q$ & Quality factor of a resonator \\
\hline$R_{C}$ & Final curvature of coated wafer \\
\hline$R_{S}$ & Initial curvature of uncoated wafer \\
\hline$S i$ & Silicon \\
\hline $\mathrm{SiO}_{2}$ & Silicon Dioxide \\
\hline$t$ & Time \\
\hline$T$ & Temperature \\
\hline$T_{0}$ & Equilibrium temperature of beam \\
\hline$T_{\text {melting }}$ & Melting temperature of sputter deposited film \\
\hline$\Delta W$ & Ratio of energy dissipated in one cycle of vibration \\
\hline$W_{\max }$ & Maximum energy stored during one cycle \\
\hline$x, y$ & Cartesian co-ordinates \\
\hline$z$ & Analytic function \\
\hline
\end{tabular}

\section{Greek Symbols}

$\begin{array}{ll}\alpha & \text { Coefficient of thermal expansion } \\ \delta & \text { Logarithmic decrement, damping } \\ \Delta J & \text { Relaxation strength }\end{array}$


$\rho$

$\tau$

$\tau_{\varepsilon}$

$\tau_{\sigma}$

$\xi$

\section{Subscripts}

$b$

$c$

$f$

$R$

$S$

$U$
Relative error in a measure of damping

Eigen mode shape of a cantilevered beam (for the $\mathrm{n}^{\text {th }}$ mode)

Poisson's ratio

Natural frequency in radians per second

Damped natural frequency

Normalized frequency

Viscous damping coefficient

Density

Mean relaxation time constant

Relaxation time at constant strain

Relaxation time at constant stress

Normalized length

Base used for attaching a cantilever

Composite (film/substrate)

Thin metal film

Relaxed

Silicon beam substrate

Un-relaxed 
xviii 


\section{Introduction}

\section{Micro- and Nano-mechanical Resonators}

From measuring forces at the atomic scale [Mey04] to providing high precision timing references for electronic circuits [Cam10, Ngu07], micro and nano electromechanical resonators (MEMS and NEMS) are a new class of devices that are providing unprecedented capabilities for the advancement modern science and technology. The term micro or nano refers to the dimensions of the resonator which can range from hundreds of micrometers down to tens of nanometers. These resonators are mechanical structures such as beams, plates, rings and discs that are harmonically excited in one of their resonant modes. Typically, the frequency of operation, the amplitude of their mechanical response, the damping and the phase lag are the main characteristics utilized for all applications [Cam10]. Examples of several devices reported in the literature are shown in Figure 1.1.

At present, commercially fabricated micro and nanometer scale mechanical resonators, in the form of cantilevered beams (Figure 1.1a), are routinely used for atomic force microscopy [Mey04, Alb91], chemical sensing [Lan02, Boi11] and biomolecular detection [Lav04]. Finely tuned experimental setups utilizing such resonators have been able to measure forces as low as $10^{-19} \mathrm{~N}$ [Mam01] and

masses as small as $10^{-18} \mathrm{~g}$ [Yan06]. The use of microscale disk and ring resonators (Figure 1.1b) as signal filters, duplexers and timing references is actively being pursued for applications in radio-frequency communication (such as cellphones and GPS units) [Cla05, Ngu07, Cam10]. Other examples of emerging applications include piezoelectric microcantilevers to convert low frequency ambient vibrations into electrical energy [Bee06, Ral10], nanoscale self sensing piezoresistive cantilevered force sensors (Figure 1.1c) and nanowire based sensors (Figure 1.1d) as highly sensitive detectors for operation under ambient pressures [Hua05]. 

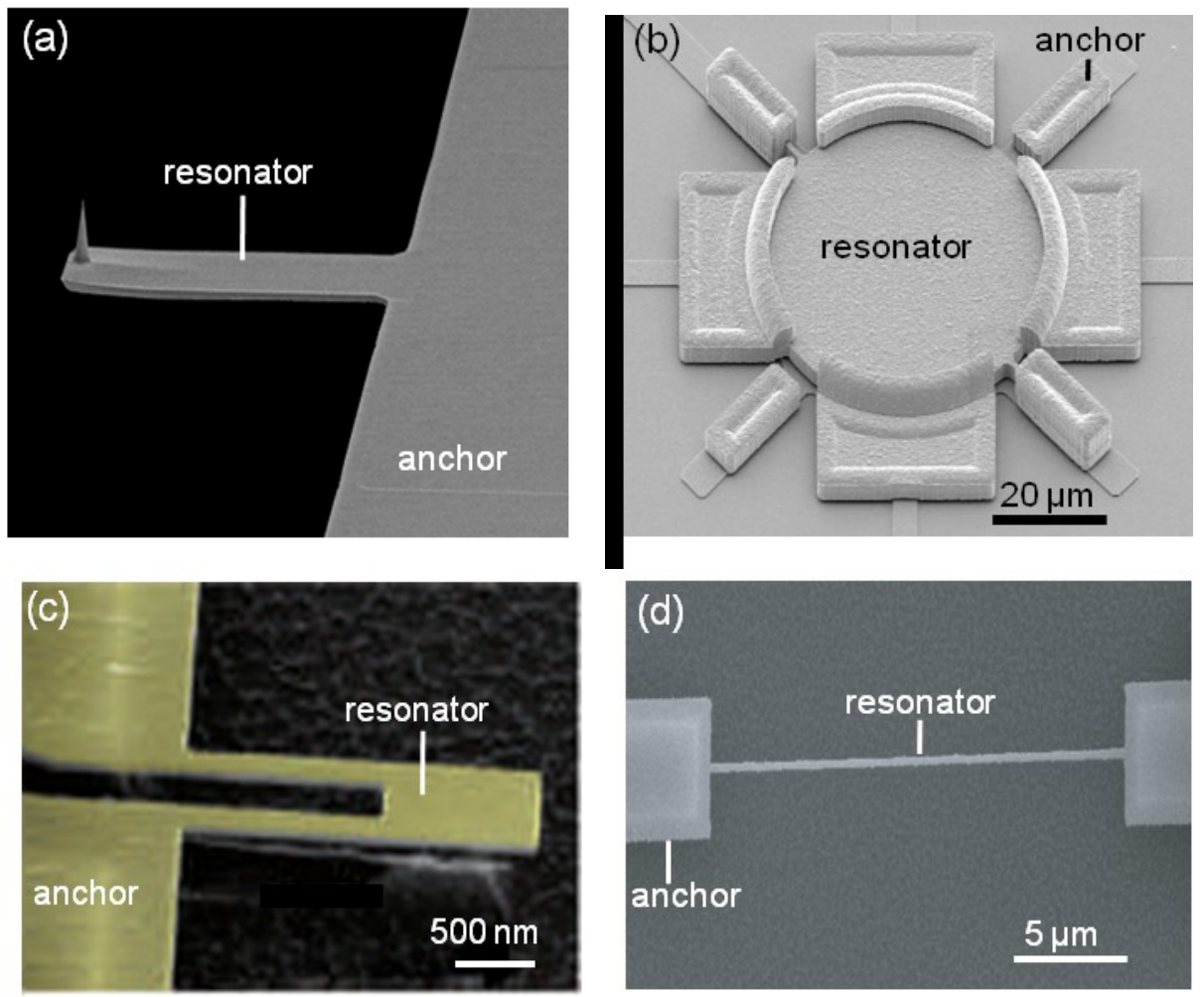

Figure 1.1: Scanning electron micrographs of common MEMS/NEMS resonators and anchor geometries from the literature a) a typical commercial silicon microcantilever used for atomic force microscopy [AIST-NT Inc] b) wine glass mode disc resonator fabricated from doped Si used as a timing reference [Ngu07], c) $100 \mathrm{~nm}$ thick cantilever used for force sensing. The cantilever is fabricated from $\mathrm{SiC}$ and coated with a $30 \mathrm{~nm}$ thick Au film. [Li07] d) $\mathrm{SiC}$ nanowire based clamped-clamped resonator used for force sensing [Hua05].

As these various microresonator devices gain maturity and make the transition from proof-of-concept demonstrations to full-fledged commercialization, there is a growing need for developing rational design methodologies to improve the performance of these devices. A survey of resonant microdevices (Table 1.1) shows a wide range of structural materials, resonant configurations, and resonating modes that are available for selection. 
Table 1.1: Design and operating parameters of micro/nanomechanical resonators [Ven08].

\begin{tabular}{|c|c|}
\hline Design Parameter & Range of Parameter \\
\hline Structural materials & Ceramics, metals, polymers, composites \\
\hline Frequency of operation & $100 \mathrm{~Hz}-10 \mathrm{GHz}$ \\
\hline Loss factor $\left(Q^{-1}\right)$ & $10^{-6}-10^{-2}$ \\
\hline Smallest dimensions & $10 \mathrm{~nm}-100 \mu \mathrm{m}$ \\
\hline Resonating structures & $\begin{array}{l}\text { Cylindrical nanowires } \\
\text { Rectangular beams } \\
\text { Plates } \\
\text { Discs } \\
\text { Rings }\end{array}$ \\
\hline Resonating modes & $\begin{array}{l}\text { Flexural, Longitudinal, Torsional } \\
\text { Bulk acoustic modes } \\
\text { Surface acoustic modes } \\
\text { Wine-glass mode } \\
\text { Contour mode }\end{array}$ \\
\hline
\end{tabular}

Although the specific details of the resonator depend on the application, they share some common characteristics, as listed below.

i. Silicon based ceramics (single crystal silicon, silicon carbide, silicon dioxide) and polycrystalline face centered cubic metal films (aluminum, gold, copper) are the most common structural materials. These materials leverage microfabrication processes that are well established in the semiconductor industry

ii. Most micro and nanoscale resonators are designed for operation at room temperature and a smaller set is designed for operation at cryogenic temperatures. $(<1 \mathrm{~K})$.

iii. Flexural beam resonators, with rectangular cross sections and ranging in thickness from $100 \mathrm{~nm}$ to $100 \mu \mathrm{m}$, are currently the most widely used geometries for micro and nanoscale resonators. 
Across all these applications, one of the most important parameters that dictates the performance of the resonator is the loss factor, $Q^{-1}$ (inverse of the quality factor, $Q)$. For most applications, designers seek to reduce the damping in microresonators and attain very low damping factors $\left(10^{-6}<Q^{-1}<10^{-4}\right)$. This critical requirement is motivated by multiple considerations: low loss factors enhance the sensitivity and signal to noise ratio of resonant sensors [Gab93, Bra93]; improve the frequency selectivity and reduces the power consumption of devices used in RF applications [Ngu07]; and increase the efficiency of vibration energy harvesters [Bee06]. However, designing a resonator for low damping is difficult. To illustrate this point, let us focus the discussion to examine the role of damping in the ultimate force sensitivity of cantilevered resonators used in atomic force microscopy.

\section{Illustrative example: The role of damping in AFM cantilevers}

The atomic force microscope (AFM) is one of the most versatile and widely used tools to characterize surface topography at the nanometer scale. At the heart of the system is a silicon micro-cantilever with a sharp tip extending from the free end (Figure 1.1a). When used in a non-contact mode (frequency modulated-AFM), the cantilever is oscillated at its resonance frequency at a small distance from the surface. This microresonator is then brought closer to the surface, where it is subjected to force gradients arising from its interaction with the surface. The effect of these forces is measured as a shift in the resonance frequency of the microcantilever [Alb91]. Measuring these frequency shifts as the cantilever is rastered over the surface provides a three dimensional image of the surface forces.

The ultimate limits on force sensitivity are dictated by the smallest shifts in frequency that can be measured. This ultimate limit is set by the thermomechanical noise in the cantilever [Alb91, Smi95, Yas00, Gab93]. In turn, this noise is fundamentally coupled to dissipation and damping in the structure, arising as a consequence of the fluctuation-dissipation theorem [Cal51, Rei65]. As the microcantilever oscillates, it is subjected to random excitation from its interaction with particles in the heat bath. In effect, the heat bath acts as a random 
driving force inducing small thermo-mechanical displacements in the cantilever. The magnitude of this thermal driving force is given by [Yas00, Bra93]

$$
F_{t h}=\sqrt{\frac{4 k_{c} k_{b} T B}{\omega_{0}} Q^{-1}},
$$

where $k_{\mathrm{c}}$ is the equivalent stiffness of the cantilever, $\omega_{0}$ is the resonant frequency of the cantilever, $k_{\mathrm{b}}$ is the Boltzmann constant, $T$ is the ambient temperature of operation, $B$ is the frequency bandwidth over which the resonator operates and $Q^{-1}$ is the loss factor. In effect, this thermal driving force establishes an absolute lower limit on forces that can be detected; Equation (1.1) therefore represents the ultimate force sensitivity of the device [Bra93]. We observe from this expression that, for any specified stiffness and natural frequency, the sensitivity can be enhanced by decreasing the damping factor.

At present, beam resonators can be tailored to operate at a specific frequency with the appropriate selection of geometry, structural materials and using accurate models that predict the dynamic behavior of the structure [Gra75, Wea90]. In contrast, designing for low damping is more difficult. The loss factor is defined as the ratio of energy dissipated in one cycle of vibration $(\Delta W)$ to the maximum energy stored $\left(W_{\max }\right)$.Alternately, the damping can be specified in terms of the viscous damping factor, $\zeta$, or the logarithmic decrement, $\delta$, of the resonator. For low values of dissipation [Gra92]:

$$
\delta=2 \zeta=\pi Q^{-1}=\frac{\Delta W}{2 W_{\max }}, \text { for } \delta<0.01
$$

(This relationship is central to the measurements in this thesis and will be derived in Chapter 2)

The damping in a resonator is a function of several extrinsic mechanisms (including viscous damping, clamping losses, and support losses) and intrinsic mechanisms (internal friction and thermoelastic losses). We shall examine these 
mechanisms in greater detail in the next chapter; for now, we can proceed by noting that considerable progress has been made in the design of vacuum operated monolithic ceramic resonators with very low damping $\left(10^{-6}<\delta<10^{-4}\right)$. In fact, resonators fabricated using single-crystal silicon, polysilicon, silicon nitride and silicon-germanium have been shown to operate at low values that approach the fundamental levels of dissipation established by thermoelastic damping (TED) [Ros90, Rei06, Har99, Fou06, Met09, Duw03, Sri02, Abd06].

For many applications, it is common practice to coat these resonators with a thin metal film to increase the electrical conductivity, optical reflectivity or to alter the surface chemistry. For instance, thin metallic films of aluminum are routinely added to cantilevers used in AFM to improve their optical reflectivity; thereby improving the signal to noise ratio of the detection system. The metallic films are significantly thinner than the underlying ceramic and have little impact on the stiffness of the structure. However, there is growing evidence to show that these coatings can lead to a substantial increase in damping, sometimes by over an order of magnitude [Sos10, Evo00, Sek02, San05].

These observations raise two important questions.

- What is the contribution to damping from these thin films?

In order to design resonators with low dissipation, designers need a catalog of experimental results to guide material selection or accurate models that predict damping in metallic thin films. Unfortunately, neither catalog nor models are available at present. As a first step towards developing these guidelines, accurate measurements of the effects of thickness, frequency, temperature and microstructure on damping are required for the range of commonly used materials.

- What are the underlying mechanisms that result in the large dissipation? Careful measurements of damping in thin film materials can provide valuable insight into the effects of size and confinement on their mechanical behavior. The damping due to these thin films is commonly termed internal friction, which is a 
function of the density, distribution and mobility of crystallographic defects. These defects include vacancies, substitutional and interstitial impurities, surface defects, dislocations, grain boundaries, and phase boundaries. The analysis of the frequency and temperature dependence of internal friction is called mechanical spectroscopy [Now72] and has been extensively used to characterize the mechanisms of dissipation in bulk solids. In stark contrast to the vast and growing literature on most other aspects of their mechanical behavior [Sur03], very little is known about internal friction in thin films. Developing techniques for mechanical spectroscopy of deposited thin films can fill this gap in knowledge.

These considerations motivate the work that is presented in this thesis. The primary objective of this works is the design and development of a technique for accurate measurement of structural damping due to internal friction in thin film materials.

\subsection{Outline of this dissertation}

The remaining chapters of this dissertation are organized as follows. Chapter 2 critically reviews the literature on measuring internal friction in deposited thin films and highlights the limitations of current approaches. Subsequently, a new approach based on a silicon microcantilever platform is presented. This approach uses the fundamental limits of thermoelastic damping to enable unambiguous calibration of internal friction in thin films. The design and development of this platform are described in detail. This chapter concludes with the demonstration of single-crystal silicon microcantilevers that operate with low damping approaching the limits of TED.

Chapter 3 then utilizes this platform to measure internal friction in sputter deposited films of aluminum, silver and gold. These three materials are widely used as functional coatings in microresonators. The processing and characterization of these films is described in detail, with particular attention to the control of growth-related residual stresses. Subsequently, a detailed study on the effects of film thickness $(60-450 \mathrm{~nm})$ and frequency $(0.1-1.5 \mathrm{kHz})$ on 
internal friction in these films is presented. The implications of these results for the design of resonators are discussed. Chapter 4 then extends the measurement of damping in $60 \mathrm{~nm}$ thick films of aluminum over a larger range of frequencies (70 $\mathrm{Hz}$ to $40 \mathrm{kHz})$.

Chapter 5 presents a strategy to control the damping in composite resonators by selectively coating the films in regions of low strain. A simple model is developed to predict the damping in partially metalized cantilevers and experimentally validated. A detailed comparison of the predictions of this model with the experiments on aluminum-coated silicon microcantilevers is presented.

Chapter 6 presents the preliminary design and testing of an experimental setup to measure damping as a function of temperature. Finally, the results from the thesis are compiled to provide a number of guidelines for the design of low damping flexural resonators and a discussion of future opportunities and challenges. 


\subsection{Contributions of this dissertation}

The original contributions of this work are as follows:

i) The design and development of a silicon microcantilever based platform for accurately measuring internal friction in thin films using thermoelastic damping as the basis for calibration. The platform is demonstrated to be capable of measuring internal friction in metal films as thin as $15 \mathrm{~nm}$ and over a frequency range from $70 \mathrm{~Hz}-40 \mathrm{kHz}$, at room temperature.

ii) The first measurements of the effects of thickness and frequency on internal friction in thin films of sputter deposited aluminum, silver and gold at room temperature. The films ranged in thickness from 60 to 450 $\mathrm{nm}$ and were studied over a frequency range from 100 to $1500 \mathrm{~Hz}$. The results from this work provide useful guidelines for designing layered resonators: gold leads to a smaller damping than either silver or aluminum and the damping in the composite resonator can be decreased by reducing the thickness of the film

iii) This thesis develops an analytical model to predict the effects of selective patterning of thin films on the damping in silicon/film composite cantilevered resonators. The model was shown to be in good agreement with experimental measurements. The model enables a design based approach to minimize the dissipative impact of thin films by selective metallization in regions of low strain. 


\section{Chapter 2}

\section{An Approach to Measuring Damping in Thin Functional Films Using Thermoelastic Damping for Calibration}

\section{Overview}

This chapter presents a new approach to the measurement of internal friction in thin film materials. Subsequently, the detailed design and development of a silicon microcantilever based platform to make damping measurements is described in detail. The chapter concludes with the demonstration of single-crystal silicon microcantilevers operating at the fundamental limits imposed by thermodynamics.

\subsection{The vibrating reed approach}

The earliest attempts to measure internal friction in thin films can be traced back to the 1960s. In 1963, Weiss and Smith suggested the use of a composite structure consisting of a thin film deposited on a low-loss quartz ribbon to measure internal friction in the film [Wei63]. Building upon this idea, Berry and Pritchet published a series of influential papers in which they described a vibrating reed apparatus (consisting of thin films deposited on miniaturized cantilevers) and established a protocol for measurement and analysis of internal friction [Ber75, Ber76, Ber81, Ber83, Ber90]. The essential features of this protocol, which has now become the standard in this field, can be summarized in a sequence of four steps. The first step is to select a substrate material that is known to have low structural damping;

Parts of this chapter appear in a peer reviewed journal publication: G Sosale, S. Prabhakar, L. Frechette, and S. Vengallatore, "A microcantilever platform for measuring internal friction in thin films using thermoelastic damping for calibration," Journal of Microelectromechanical System., vol. 20, pp.764-773 (2011) 
single-crystal silicon and quartz are widely used. Next, this material is machined to create a cantilevered beam of thickness $h_{s}$, taking care to design the supports and clamps to minimize any frictional losses. Crucially, these support and clamping losses are not quantified in the standard protocol; therefore, they are not demonstrated to be negligible. We shall return to this important point later in the chapter.

The third step is to measure the logarithmic decrement of the bare substrate, $\delta_{s}$, by recording the decay of the amplitude of free vibrations. All measurements are performed at sufficiently low pressures to ensure that viscous losses due to air damping or squeezed film damping are negligible. The fourth step is to deposit the film of interest and measure the damping of the composite film-substrate system, $\delta_{c}$.

At this point, it is necessary to confront the difficult question of extracting the internal friction in the film from the damping measured in the substrate and bilayer. To this end, Berry and Pritchet suggested a layer-by-layer partitioning of the dissipated energy so that [Ber75]

$$
\delta_{c}=\frac{\Delta W_{c}}{2 W_{c, \max }}=\frac{\Delta W_{s}}{2 W_{c, \text { max }}}+\frac{\Delta W_{f}}{2 W_{c, \text { max }}} .
$$

In this expression, and in what follows, the subscripts $c, s$, and $f$ denote the composite, substrate, and film, respectively. Equation (2.1) assumes that the film is perfectly attached to the substrate. In the limiting geometry of a thin film on a thick substrate with $h_{i} \leq 0.01 h_{s}, W_{c, \eta_{i s i . r}} \approx W_{s, \eta_{i s i . r}}$ [Ber75]; therefore, Equation (2.1) can be expressed as

$$
\delta_{c}=\frac{\Delta W_{s}}{2 W_{s, \max }}+\frac{\Delta W_{f}}{2 W_{s, \max }} .
$$

The first term on the right hand side can be identified as the logarithmic decrement of the bare substrate. The second term can be suitably normalized to 
obtain the logarithmic decrement of the film if it were to be measured independently under the same conditions [Ber75]. Thus,

$$
\delta_{c}=\delta_{s}+\frac{W_{f, \text { max }}}{W_{s, \text { max }}}\left(\frac{\Delta W_{f}}{2 W_{f, \text { max }}}\right)=\delta_{s}+\left(\frac{W_{f, \text { max }}}{W_{s, \text { max }}}\right) \delta_{f}=\delta_{s}+\left(3 \frac{E_{f} h_{f}}{E_{s} h_{s}}\right) \delta_{f},
$$

where $E$ is the Young's modulus. The term in the parenthesis is the ratio of the maximum stored elastic energy in the film and substrate. This ratio can be expressed in terms of the elastic modulus and thickness of the film and substrate by a standard analysis of the quasi-static deformation of an Euler-Bernoulli beam [Ber75].

Equation (2.3) has been used in numerous studies to extract the value of $\delta_{f}$ from the measured values of $\delta_{c}$ and $\delta_{s}$ [Ber75-90, Boh92, Su94, Pri94, Fuj95, Pe195, Nis04, Nis06, Cho06, Cho04, Hag02, Zhu95]. Further, $\delta_{f}$ is commonly identified as the internal friction caused by the motion of crystallographic defects in the film. This identification, unfortunately, is not accurate. Each of $\delta_{c}, \delta_{f}$ and $\delta_{s}$ contains contributions from several mechanisms of dissipation, and an error of unknown magnitude is incurred if $\delta_{f}$ is taken as the estimate of internal friction. This feature of the standard protocol has been noted before. In 1992, Bohn and Su suggested that Equation (2.3) must be modified to incorporate an additional term to account for these other sources of dissipation, which they termed background damping (BG) [Boh92]; hence,

$$
\delta_{c}=\frac{\Delta W_{c}}{2 W_{C, \max }}=\delta_{s}+\frac{3 E_{f} h_{f}}{E_{s} h_{s}} \delta_{f}+\delta_{B G}
$$

However, no systematic method has yet been proposed to quantify this background damping. Moreover, some mechanisms of dissipation, including thermoelastic damping in the bi-layer, cannot be easily partitioned layer-by-layer [Ven05]. To resolve all these long-standing problems, a new approach is proposed based on an explicit mechanism-by-mechanism partitioning of $\delta_{c}$. The essential 
idea underlying this approach is the use of thermoelastic damping for calibrating measurements of internal friction, as discussed in the next section.

\subsection{Theory: calibration using thermoelastic damping}

Consider a slender, straight cantilevered beam of solid rectangular cross-section with thickness $h_{s}$ and length $L$, as shown in Figure (2.1). The beam, which is free of residual stress, is micromachined such that it is attached monolithically to a larger supporting structure which, in turn, is clamped to the package or shaker. The beam and shaker assembly are located within a vacuum chamber that is maintained at a sufficiently low pressure to make viscous air damping negligible. The beam undergoes small-amplitude bending vibrations, and the structural damping can be measured from the free decay of bending vibrations and expressed in terms of the logarithmic decrement, $\delta_{s}$. Synthesizing results from several theoretical and experimental investigations of damping in vacuumoperated microcantilevers [Sos10, Yas00, Li07, Ros90, Rei06, Har99, Fou06, Met09, Duw03, Sri02, Abd06] the measured damping can be partitioned into clamping losses, support losses, internal friction (IF) and thermoelastic damping (TED). Hence,

$$
\delta_{s}=\delta_{s, \text { clamp }}+\delta_{s, \text { sup } p o r t}+\delta_{s, I F}+\delta_{s, T E D} .
$$

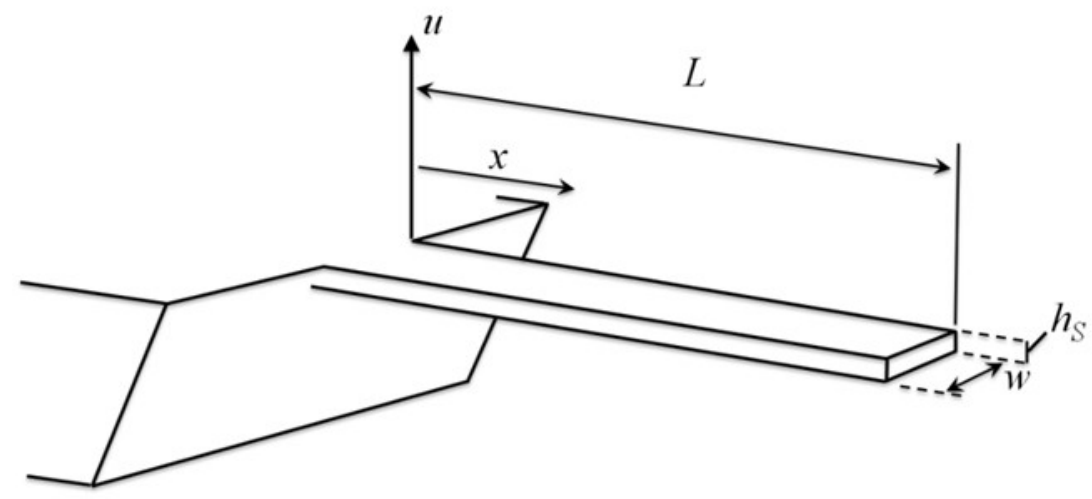

Figure 2.1: Schematic of a microfabricated silicon cantilevered beam attached to a large supporting structure. 
The clamping losses are due to friction at the points of attachment of the vibrating structure to the test apparatus. This dissipation is difficult to quantify because it depends on numerous local variables at the region of contact. However, clamping losses can be minimized by following guidelines that have been developed based on experience. For instance, incorporating a step at the base of the cantilever can minimize losses due to stick-slip friction [Rei06]. The second source of dissipation is due to the interaction of the vibrating beam with the larger supporting structure. As the beam vibrates, it applies a time-harmonic force on the support, thereby leading to the generation and propagation of elastic stress waves into the support. Typically, the majority of this energy is not reflected back into the cantilever. Therefore, this mechanism of elastic stress wave generation is effectively a source of dissipation. Analysis of an idealized geometry consisting of a cantilever attached monolithically to an elastic half-space predicts that the support loss scales as $\left(h_{s} / L\right)^{3}$ [Hos95, Hao03]; therefore, these losses can be made negligible by employing long slender beams with high aspect ratio of beam length to thickness. However, this scaling can change for more realistic geometries of micromachined cantilevers [Pho04], and detailed numerical analysis may be required to estimate support losses. Alternately, more sophisticated designs can be employed to isolate the beam from the supports by creating free-free beams that are attached at their nodal points [Fer05]. These considerations will be addressed in more detail in the next section.

The third source of dissipation is due to internal friction caused by the motion of crystallographic defects within the cantilever. Computing the magnitude of internal friction requires knowledge of the type, density and distribution of various crystallographic defects, and their mobility within the structure [Now72, Bla07]. Even when such detailed information is available, it is not always trivial to develop models that can predict the magnitude of internal friction. However, a simple strategy for minimizing internal friction is to use a high-quality singlecrystal material, such as commercially-available single-crystal silicon, that is known to possess a very low density of crystallographic defects. 
The fourth source of dissipation is thermoelastic damping (TED). This mechanism of structural damping is operative in every flexural resonator with a non-zero thermal expansion coefficient. We must consider this mechanism in some detail because this source of dissipation plays a significant role in the approach developed in this thesis.

TED is the result of irreversible heat conduction across thermoelastic temperature gradients in beams undergoing bending vibrations. To illustrate this point, let us consider the beam shown in Figure 2.1 as it executes small, transverse, flexural oscillations. During one half cycle of oscillation, the stress distribution across the cross section of the beam is such that one half of the beam is in tension, the other in compression. Half a cycle later, this stress state is reversed. For a solid with a positive thermal expansion coefficient, the stress field creates a corresponding temperature field. As a result of thermoelastic coupling, a solid undergoing adiabatic and reversible deformation heats up when in compression and cools down in tension [Tho78]. Therefore, at any instant, the half of the beam in tension is slightly cooler and the other half in compression is slightly warmer. This results in irreversible heat conduction along this minute temperature gradient. In turn, the heat conduction leads to entropy generation and energy dissipation, as first identified by Zener in 1937 [Zen37, Zen38]. Therefore, regardless of any other consideration, TED must operate in all beams undergoing bending vibrations. In that sense, TED establishes the absolute lower bound on structural damping in the microcantilever. The frequency dependence of TED can be calculated using a simple but approximate formula developed by Zener [Zen37],

$$
\delta_{s, T E D}=\frac{\pi E_{s} \alpha_{s}^{2} T_{0}}{C_{s}} \frac{\Omega}{1+\Omega^{2}} ; \Omega=\omega \tau_{T E D}=\omega \frac{C_{s} h_{s}^{2}}{\pi^{2} k_{s}}
$$

In this expression, $\alpha$ is the coefficient of thermal expansion, $T_{0}$ is the equilibrium temperature of the beam, $C$ is the specific heat per unit volume, $\omega$ is the angular frequency (in radians/second), $\tau$ is a time constant, $k$ is the thermal conductivity, and $\Omega$ is the normalized frequency. As shown in Figure (2.2), $\delta_{s, T E D}$ has a single peak and attains its maximum value when $\Omega=\omega \tau_{T E D}=1$. This graph was 
calculated using the following material properties of single-crystal silicon: $E=$ $169 \mathrm{GPa}, \alpha=2.6 \times 10^{-6} \mathrm{~K}^{-1}, C=1.6 \times 10^{6} \mathrm{~J} / \mathrm{m}^{3} / \mathrm{K}$, and $k=149 \mathrm{~W} / \mathrm{m} / \mathrm{K}$ [Rei06].

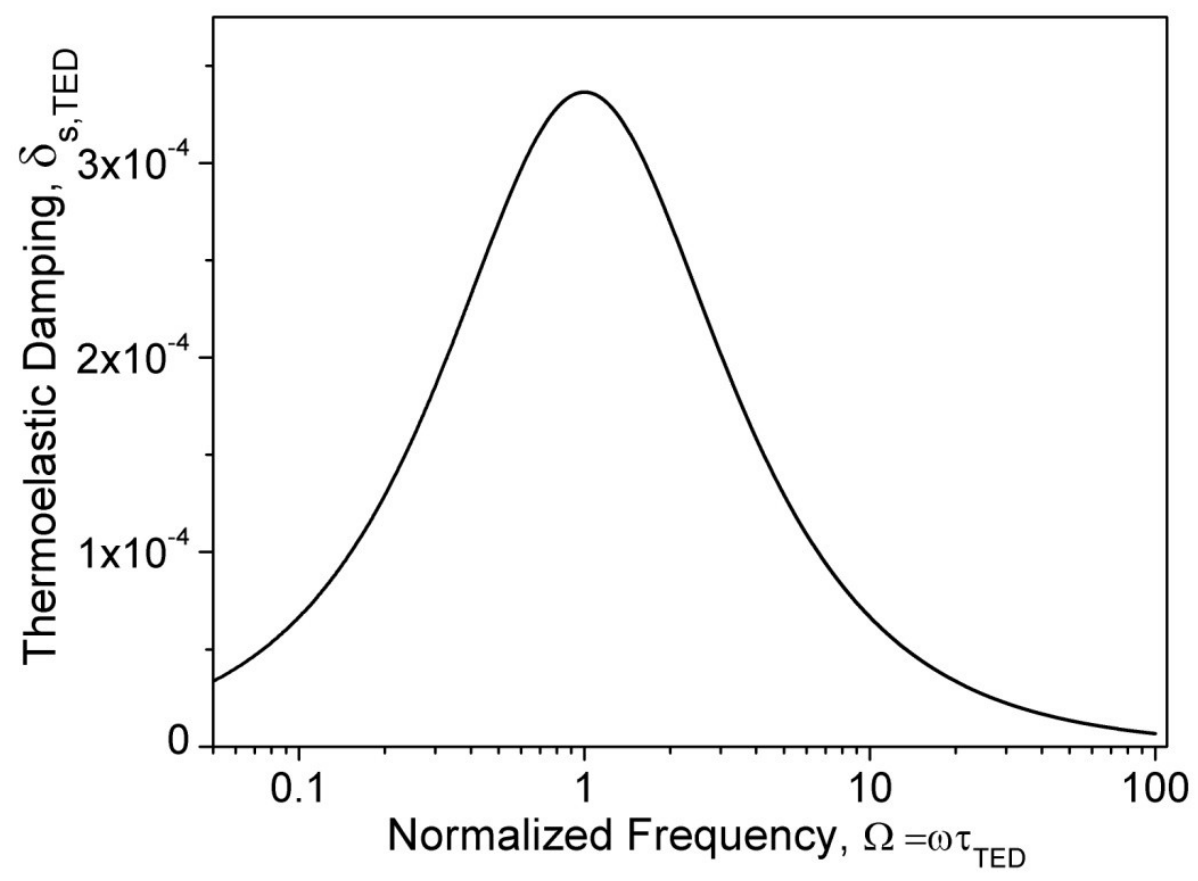

Figure 2.2: Graph of thermoelastic damping as a function of the normalized frequency for silicon beams calculated using Equation (2.6).

Equation (2.6) does not contain any free parameters. Detailed one-dimensional [Kin94, Lif00] and two-dimensional [Pra08] models have shown that Zener's formula is accurate to within a few percent for structures of practical interest. Therefore, given the material properties and the thickness of the beam, we can calculate thermoelastic dissipation at any specified frequency. A comparison of the measured damping, $\delta_{s}$, with the predictions of Equation (2.6) can establish whether the beam is operating at the fundamental limit of dissipation established by TED.

Equation (2.5) is valid for all microcantilevers. Let us now specialize this equation for the case of single-crystal silicon microcantilevers with carefullydesigned supports and clamps. To the extent that internal friction, support losses and clamping losses have been made negligible, we expect this class of beams to 
operate at the limit of dissipation established by TED. In practice, it is currently possible to reduce the combined contributions of internal friction, support losses and clamping losses below $10^{-5}$ at room temperature [Sos10]. Hence, Equation (2.5) can be expressed as

$$
\delta_{S}=\delta_{s, T E D}+O\left(10^{-5}\right)
$$

Thus, silicon beams that follow Equation (2.7) can be used to measure internal friction in deposited thin films with accuracy on the order of $10^{-5}$. To accomplish such measurements, we consider the deposition of a film of thickness $h_{f}$ on the silicon beam. If necessary, thin adhesion layers $(\mathrm{Ti}, \mathrm{Ta}$, and $\mathrm{Cr}$ with thickness of $\sim 10 \mathrm{~nm}$ ) can be used to ensure good adhesion between the film and silicon [Ohr02]. The measured damping of this layered beam is the sum of TED in the composite and internal friction in the film. Hence,

$$
\delta_{c}=\delta_{c, T E D}+\frac{3 E_{f} h_{f}}{E_{s} h_{s}} \delta_{f, I F}+O\left(10^{-5}\right) .
$$

The first term on the right hand side captures the increase in TED due to the addition of the thin film. The frequency dependence of $\delta_{c, T E D}$ can be computed accurately using a model developed by Prabhakar and Vengallatore [Pra05] that accounts for heat conduction across the thickness of the composite beam. This model leads to an expression for TED in the form of an infinite series. Although this expression is more tedious to evaluate than Equation (2.7), it shares all the essential features of Zener's formula, namely, there are no free parameters, and TED can be computed using only the material properties (Young's modulus, coefficient of thermal expansion, specific heat, and thermal conductivity of the two materials) and the thickness of the two layers as input. Using this model, they found that the increase in TED due to the film cannot always be ignored even in the limit of a thin film on a thick substrate. As an example, the peak TED in a 100 $\mu \mathrm{m}$ thick single-crystal silicon beam is $3 \times 10^{-4}$. If this beam is now coated with a 1 $\mu \mathrm{m}$ thick film of nickel, the TED increases to $4 \times 10^{-4}$. This increase in damping is 
an order of magnitude larger than the resolution with which we seek to measure internal friction in the films. In fact, the increase in TED due to the coating of common metals ( $\mathrm{Al}, \mathrm{Au}, \mathrm{Ni}, \mathrm{Cu}, \mathrm{Ag}$ ) on single-crystal silicon is negligible only if $h_{f} \leq 10^{-3} h_{S}$. For such ultrathin films, $\delta_{c, T E D} \cong \delta_{s, T E D}$; therefore, Equation (2.8) can be expressed as

$$
\delta_{c}=\delta_{s, T E D}+\frac{3 E_{f} h_{f}}{E_{s} h_{s}} \delta_{f, I F}+O\left(10^{-5}\right) .
$$

Equations (2.8) and (2.9) can be used to devise a new protocol to measure internal friction in thin films and ultrathin films, respectively. This protocol can be implemented using a sequence of four steps.

Step 1: Use single-crystal silicon beams that operate with low damping approaching the limit of TED.

The first step is to micro-machine single-crystal silicon beams with carefully designed clamps and supports, and to demonstrate that these beams can operate at, or close to, the limit of dissipation established by TED. This is achieved by comparing the measured damping with the predictions of Zener's formula, Equation (2.6).

Step 2: Coat the beams with the thin film of interest.

Independent measurements and guidelines based on experience are necessary to ensure excellent adhesion between the film and substrate.

Step 3: Measure the damping in the substrate-film bilayer.

The measured damping is a combination of internal friction in the beam and TED in the bilayer

Step 4: Subtract the measured damping in the substrate from the damping in the composite. 
For ultrathin films of common metals, the internal friction in the film can be obtained from Equation (2.9). All studies presented in this thesis focus on this configuration of ultrathin films on a thick substrate. For thicker films, the TED in the composite must be computed, and the internal friction in the film can be obtained by using Equation (2.8).

This four step approach forms the basis of all studies presented in this thesis. The next section describes the design and development of a silicon microcantilever platform to implement this procedure, culminating in the demonstration of a set of single-crystal silicon microcantilevers that approach the limit imposed by TED. 


\subsection{Design and development of the measurement setup}

The design of the damping measurement setup was based on the vibrating reed approach developed by Berry and Pritchet [Ber75]. The basic principle of operation is to oscillate a cantilevered beam at one or more of its resonance frequencies and extract the mechanical damping from the free decay. The primary design requirement for this setup was to operate beams ranging from 0.1 to $5 \mathrm{kHz}$ in frequency and with low values of TED $\left(\delta_{s, T E D} \leq 1 \times 10^{-4}\right)$.

A schematic of the measurement setup developed in this work is shown in Figure 2.3. At the heart of the setup is a single-crystal silicon beam that is micromachined out of a high purity, semiconductor grade wafer. This beam is clamped in a precision machined stainless steel clamp and then mounted onto a piezoelectric shaker. The beam, clamp and shaker assembly is then loaded into a vacuum chamber. The shaker is excited by a sinusoidal voltage signal prescribed by a function generator and its motion is independently monitored by a capacitative displacement sensor (CDS) built into the body.

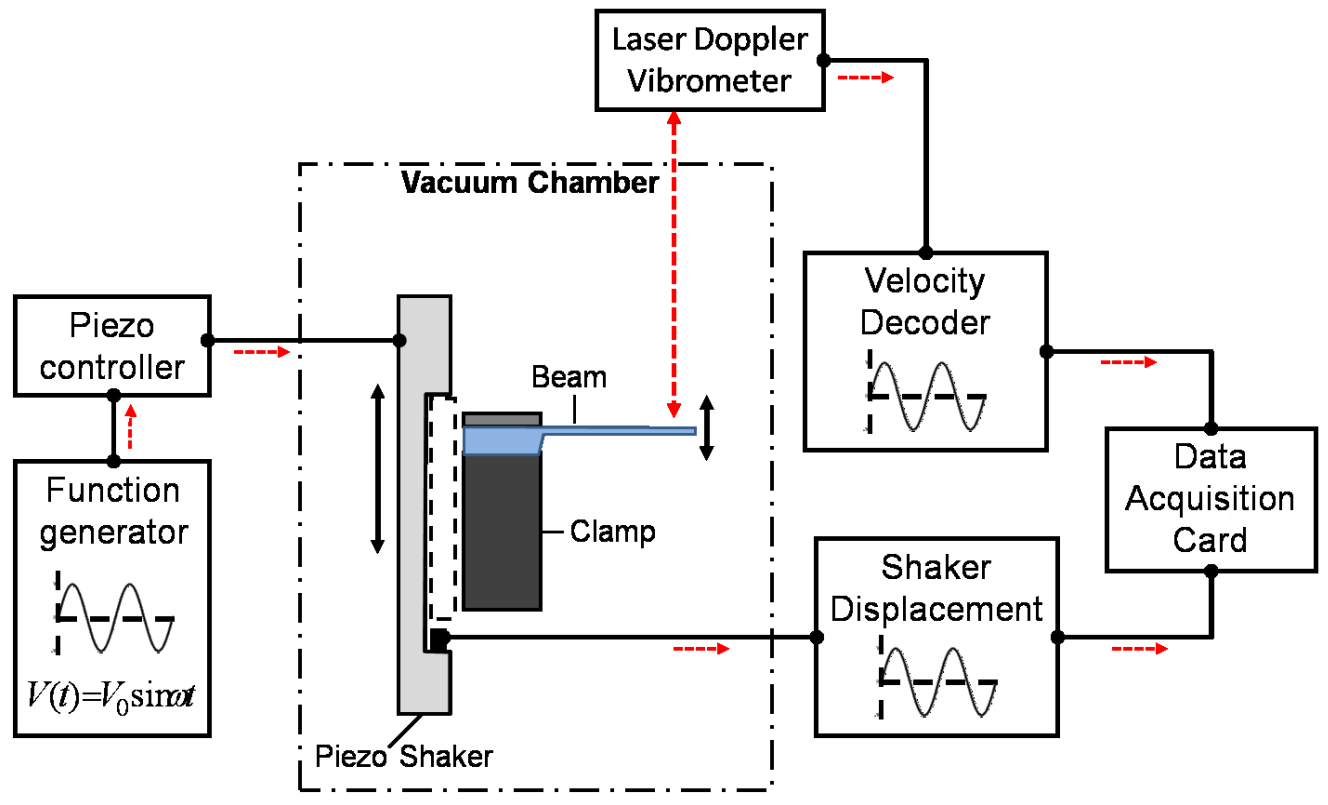

Figure 2.3: Schematic of components of the complete damping measurement system. The red dashed arrows indicate the flow of signal information. 
The motion of the beam is measured with a laser Doppler Vibrometer (LDV) that is located outside the vacuum chamber. To measure the damping, the beam is excited at its natural frequency, the excitation is terminated, and the damping is extracted from the free decay.

The biggest challenge in developing this setup was to minimize the viscous, clamping and support losses such that the beams operate close to the thermoelastic damping limit for silicon. In addition, the system had to be robust and the measurements repeatable to enable systematic parametric studies of damping using hundreds of beams. What follows is the detailed description of the design, selection, systematic testing and calibration of each of the components of this setup.

\subsubsection{Design and fabrication of single-crystal silicon specimens and clamp}

\subsubsection{Minimizing internal friction losses in the substrate}

The primary selection criteria for the substrate material were that it had to be a single-crystal material (with a low density of crystallographic defects and impurities) and, that the material could be easily fabricated into cantilevered beams with low thermoelastic damping $\left(\delta_{s, T E D} \leq 1 \times 10^{-4}\right)$ in the frequency range of $0.1-5 \mathrm{kHz}$.

Quartz crystals and single-crystal silicon [Ber75] were two candidate materials. For equivalent beam thicknesses, quartz has lower thermoelastic dissipation than silicon over the range of frequencies required here. However, micromachining quartz is difficult: few micromachining processes exist and large crystalline quartz wafers were not readily available. On the other hand, single-crystal silicon can be micromachined easily with a wide range of processes currently used in the MEMS and the microelectronics industry. Many of these processes can be implemented in the NanoTools Microfabrication Facility at McGill University. For these reasons, single-crystal silicon was selected as the substrate material. 


\subsubsection{Minimizing support losses}

Support losses arise from the coupling of the vibration modes of the resonator to the supports. Energy is dissipated in the form of elastic waves that are radiated into the support at the attachment points and are not reflected back into the beam. Hao et al provided an estimate for this loss by modeling a microscale flexural resonator as a cantilevered beam attached to a semi-infinite base [Hao03]. Their work showed that the magnitude of support losses is proportional to $\left(h_{\mathrm{s}} / L\right)^{3}$. This means that specimens with length-to-thickness aspect ratios greater than 100 should experience negligible support losses $\left(\delta_{\text {Support }}<3 \times 10^{-6}\right)$ and would suffice for measurements. However, the fundamental assumption in their work is that the wavelength of the propagating transverse waves is smaller than the dimensions of the base. For the design of beams with relatively low frequencies (100 Hz-10 $\mathrm{kHz}$ ) this requirement cannot be realized in practice. For example, the speed of propagation of shear (transverse) waves in elastic solids is given by [Jud07]

$$
c=\sqrt{\frac{E}{2(1+v) \rho}} .
$$

where $E$ is the Young's modulus, $\rho$ is the density and $v$ is the Poisson's ratio. A beam attached to this solid and resonating at a frequency of $f$ would produce shear waves with wavelengths given by $\lambda=c / f$. For a single-crystal silicon resonator operating at $100 \mathrm{~Hz}$, these shear waves have a wavelength of $57 \mathrm{~m}$ which is significantly larger than any practical dimensions of the beam or base. A more realistic model should therefore remove the assumption of a semi-infinite base. Such a model was developed by Photaidis et al and Judge et al by considering a beam machined out of a base with infinite width but finite thickness as shown in Figure 2.4 [Pho04, Jud07]. 


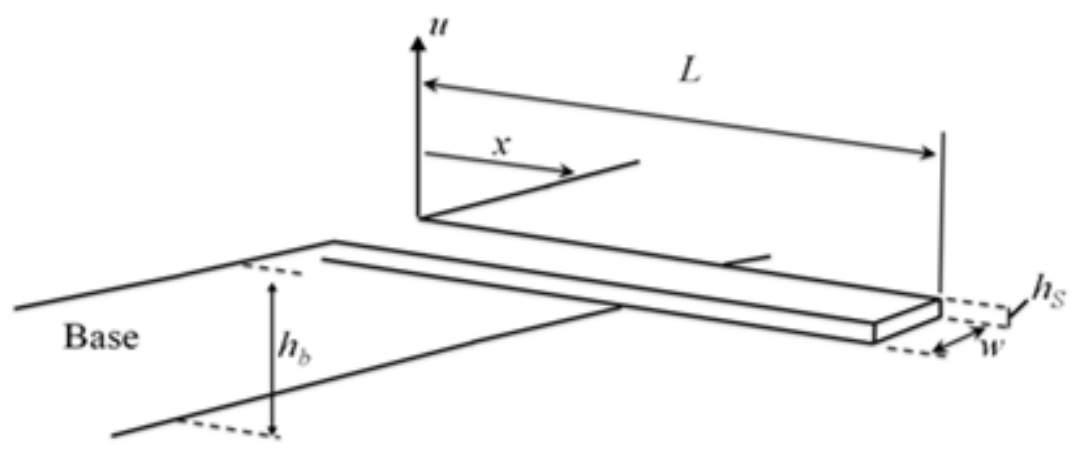

Figure 2.4: Schematic of a microfabricated silicon cantilevered beam attached to a base with a finite thickness but infinite width as considered in [Pho04].

Their calculations indicate that the dissipation in the beam is affected by the thickness of the base $h_{b}$ and scales as

$$
\delta_{\text {Support }} \propto \frac{w}{L}\left[\frac{h_{s}}{h_{b}}\right]^{2},
$$

Hence, the thicker the base, the smaller is the coupling between the beam and base and therefore the smaller the dissipation. To approach low support $\operatorname{losses}\left(\delta_{\text {Support }}<1 \times 10^{-5}\right)$ would therefore require $h_{s} / h_{b}$ of the order of $10^{-3}$. In practice these ratios are difficult to achieve for low frequency beams $(<50 \mathrm{kHz})$ using standard semiconductor grade single-crystal silicon wafers that range in thickness from 250 to $750 \mu \mathrm{m}$. Alternately, the size of the base can be effectively enlarged by clamping the beam to a much larger structure, thereby reducing support losses. This strategy, however, creates its own set of losses associated with clamping, as discussed in the next section. 


\subsubsection{Minimizing clamping losses}

\section{Geometrical design of the beam to reduce clamping loss}

One of the major losses in clamping a beam is stick-slip friction arising from the high surface strain field at the root of an oscillating cantilever. Nouira et al investigated this problem by considering the dynamics of monolithic silicon bar that was clamped at one end, in a manner similar to that illustrated in Figure 2.5 [Nou08]. By placing strain gauges at the clamped surface they were able to measure the strain at these surfaces. Their measurements showed that even with smooth controlled clamping surfaces and under vacuum conditions, the strain induced friction at the clamped surface of the beam significantly affected the dissipation (the damping in the beams was in the order of $10^{-2}$ ). This study suggests that minimizing the strain at the clamped surface is necessary to reduce the clamping loss.
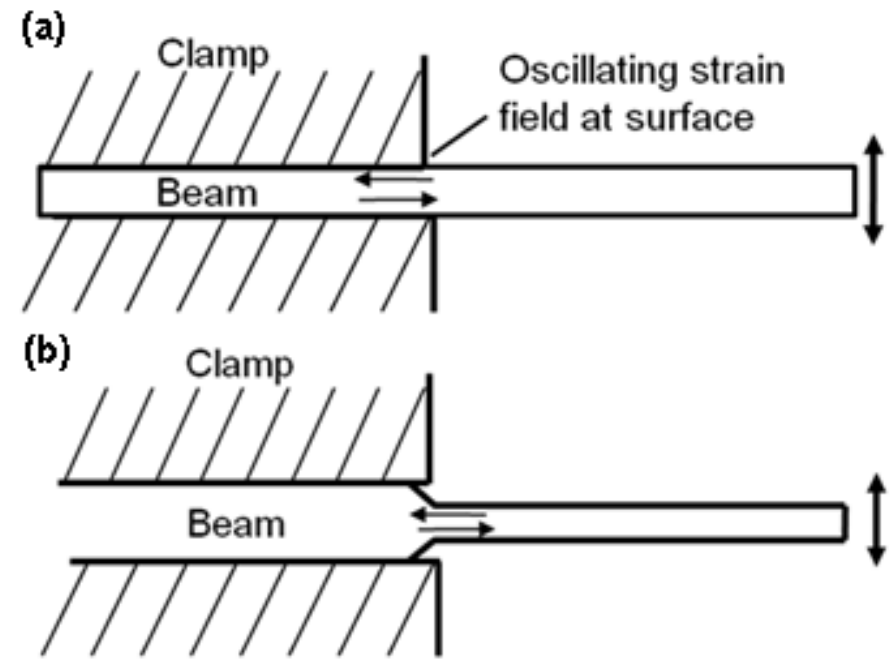

Figure 2.5: Schematic illustration of the strain field at the root of an oscillating cantilever a) at the clamped surface in a monolithic beam, b) at the root of a beam with a micromachined step.

The internal friction community has learnt by experimental trial and error that minimizing strain energy under the clamp surface is important for reducing these losses. Indeed, a wide range of specimen geometries and experimental 
configurations can be found in the literature. The main classes of designs are highlighted in Table 2.1. The common theme in these designs is to move the region of maximum strain away from the clamped surface (Figure 2.5b). We selected the geometry of Yang et al (Table 2.1(d)) in this thesis [Yan96] because of its simplicity: the notch in this design can easily be machined with simple and conventional silicon microfabrication processes.

Table 2.1: Various reed configurations used for internal friction studies.

\begin{tabular}{|c|c|c|}
\hline & Design & Comments \\
\hline (a) & & $\begin{array}{l}\text { Baur et al carried out a finite element } \\
\text { analysis of this structure showing } \\
\text { minimal strain at the base in the } 2^{\text {nd }} \\
\text { mode [Bau } 85] \text {. }\end{array}$ \\
\hline (b) & & $\begin{array}{l}\text { Börner et al used this sample to isolate } \\
\text { the strain to the narrow trapezoidal } \\
\text { section [Bor85]. }\end{array}$ \\
\hline (c) & & $\begin{array}{l}\text { Schwarz et al concentrated the strain } \\
\text { at the notch and utilized the } \\
\text { overhanging region to control the } \\
\text { frequency [Sch83]. }\end{array}$ \\
\hline (d) & & $\begin{array}{l}\text { Yang et al carried out special } \\
\text { machining on ceramic samples to } \\
\text { realize the step [Yan96]. }\end{array}$ \\
\hline (e) & & $\begin{array}{l}\text { Reid et al used a silicon-on-insulator } \\
\text { wafer to create a one sided step } \\
\text { [Rei06]. }\end{array}$ \\
\hline
\end{tabular}




\section{Fabrication of the single-crystal silicon cantilevered resonators}

In order to incorporate the step, the single-crystal silicon beams were fabricated by a standard microfabrication process. The details of this process are illustrated in Table 2.2 using two-dimensional cross-sections. In brief, the starting materials for microfabrication were single-side polished, p-type, (llll 100$)$-oriented singlecrystal silicon wafers with a thickness of $\sim 520 \mu \mathrm{m}$ and diameter of six inches. These wafers are doped with boron at a low concentration of $\sim 10^{15} / \mathrm{cm}^{3}$. First, the silicon wafers were oxidized for 26 minutes at $1100{ }^{\circ} \mathrm{C}$ to grow $\mathrm{SiO}_{2}$ films with a thickness of $\sim 500 \mathrm{~nm}$ on both surfaces. The oxide films were then patterned using a standard photolithographic process and selectively etched; these patterns served as the mask for the anisotropic wet etching of silicon using tetra methyl ammonium hydroxide (TMAH). The silicon wafer was diced parallel to [1 10 l 1 direction, and etched to produce cantilevered beams. Representative images of the entire beam and the micromachined step are shown in Figure 2.6. The beams ranged from 35 to $131 \mu \mathrm{m}$ in thickness, 0.72 to $1.1 \mathrm{~mm}$ in width, and 0.21 to 3.6 $\mathrm{cm}$ in length. The length-to-thickness aspect ratio of the beams ranged from 58 to 834 , and the fundamental frequency of bending vibrations ranged from $51 \mathrm{~Hz}$ to $10.9 \mathrm{kHz}$. Scanning electron microscopy confirmed that the variation in thickness along the axis of the beams was less than $2 \%$.

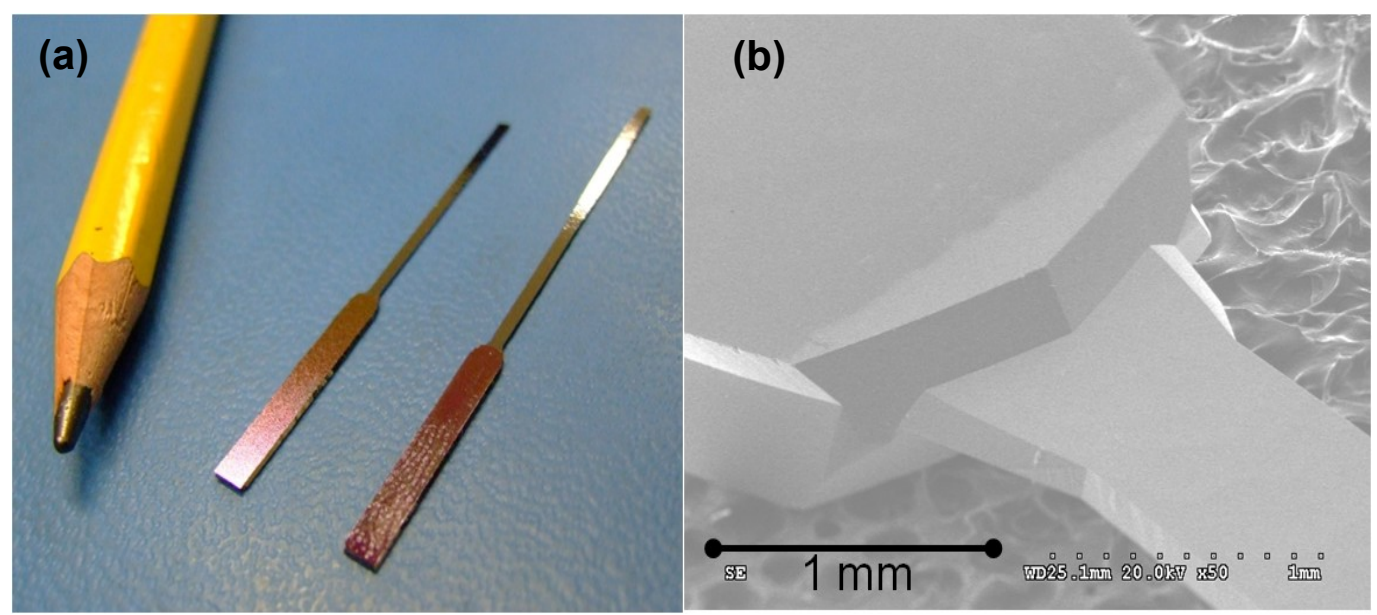

Figure 2.6: Micromachined silicon cantilevers produced by the process outlined in table 2.2. a) photograph showing the beams and their supporting structure b) magnified scanning electron micrograph of the micromachined step at the base of the cantilever. 
Table 2.2: Process flow for fabricating of low frequency single crystal silicon beams with a step at the base. The steps are represented by two dimensional cross sections that are typically used to describe microfabrication process flows. These cross-sections are not drawn to scale.

\begin{tabular}{|c|c|c|}
\hline & Process schematic & Fabrication process details \\
\hline (a) & & $\begin{array}{l}\text { Starting material: Single crystal Si }(1,0,0) \text {, } \\
\text { single side polished, } 6 ", \text { P-type, lightly } \\
\text { doped wafers with } \sim 500 \mathrm{~nm} \text { thermal oxide } \\
\text { layer grown on both sides }\end{array}$ \\
\hline (b) & & $\begin{array}{l}\text { The wafer is coated with a positive } \\
\text { Photoresist that is patterned to expose part } \\
\text { of the oxide layer underneath }\end{array}$ \\
\hline (c) & & $\begin{array}{l}\text { The exposed oxide is etched away by } \\
\text { reactive ion etching (RIE) using a } \\
\text { CHF3:CF4:Ar (40:1:70) gas plasma. }\end{array}$ \\
\hline (d) & & $\begin{array}{l}\text { The patterning is repeated on the backside } \\
\text { of wafer }\end{array}$ \\
\hline (e) & & The wafer diced into $2.75 \mathrm{~mm}$ wide strips \\
\hline (f) & & $\begin{array}{l}\text { The strips are then dipped in buffered } \\
\text { oxide etchant (BOE) to remove the native } \\
\text { oxide and etched in } 27 \% \text { TMAH at } 85^{\circ} \mathrm{C} \\
\text { to the desired thickness. The final step has } \\
54.74^{0} \text { angle to the }(1,0,0) \text { plane. }\end{array}$ \\
\hline \multicolumn{3}{|c|}{$-\mathrm{SiO}_{2}, \square \mathrm{Si} \square$ Photoresist. } \\
\hline
\end{tabular}




\subsubsection{Design of the clamp}

There were two main design requirements for the clamp. The first was that the mass of the clamp had to be maximized to prevent any coupled body motion between the clamp and beam. However, the mass had to be low enough to prevent lowering the resonances of the piezo shaker system (the details of this system are discussed in the next section). A limit of $175 \mathrm{~g}$ was imposed on the mass in consultation with the manufacturer of the shaker. This value is still a thousand times greater than the mass of typical cantilever specimens and therefore minimized any coupled body motion. The second requirement for the clamp was that the base of the cantilever should be uniformly and securely clamped to prevent any stick slip friction.

Figure 2.7 shows the final design of the clamp and its associated components. Machined out of a single block of stainless steel $(50 \times 35 \times 25 \mathrm{~mm})$, the overall weight of the clamp is approximately $175 \mathrm{~g}$ when fully assembled. In order to ensure a uniform clamping force, the clamping surfaces in contact with the base of the cantilever were machined to high tolerances for both surface roughness and parallelism. The clamping force is achieved by six screws that are sequentially tightened with a calibrated torque screw driver to a maximum torque of $1 \mathrm{Nm}$. Through numerous trials, the parallelism was found to be the most critical parameter to ensure repeatable results. Therefore, two close fitting guide pins were designed into the structure to ensure that the two clamping surfaces remain parallel as they are being tightened. The entire clamp is then attached to a mating plate (Figure 2.7(a)) that remains permanently attached to the piezoelectric shaker.

At all decision points during the design of the clamp, careful consideration was given to the repeatability of measurements (The clamp is the part of the system that would experience the most manual handling and wear) For instance, the mating plate (Figure 2.7(a)) was a design modification that was added to prevent the aluminum threads in the shaker from being worn out. The stainless steel threads in the mating plate are more resilient to repeated use; in case they are damaged, the entire shaker body need not be replaced. 


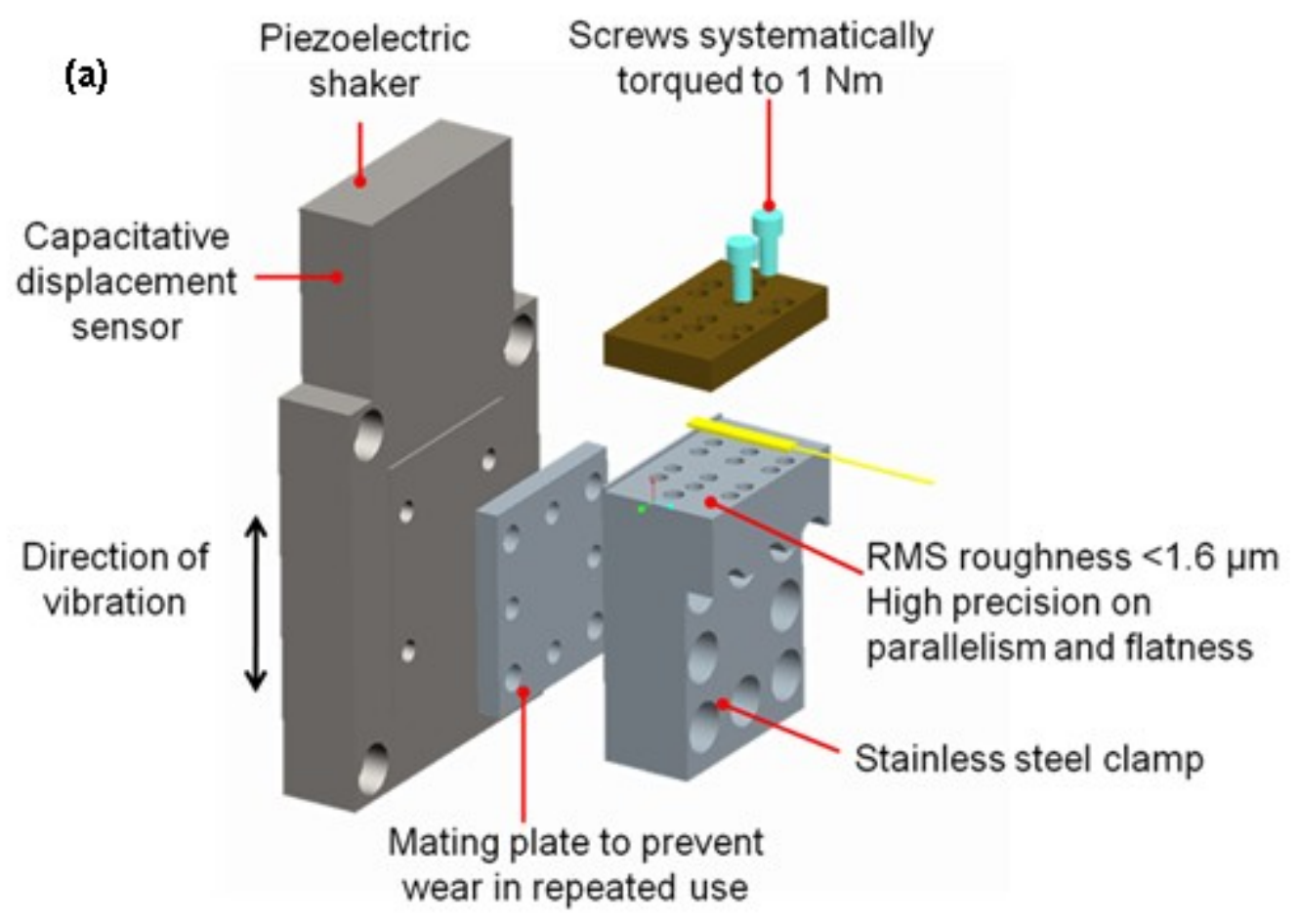

(b)

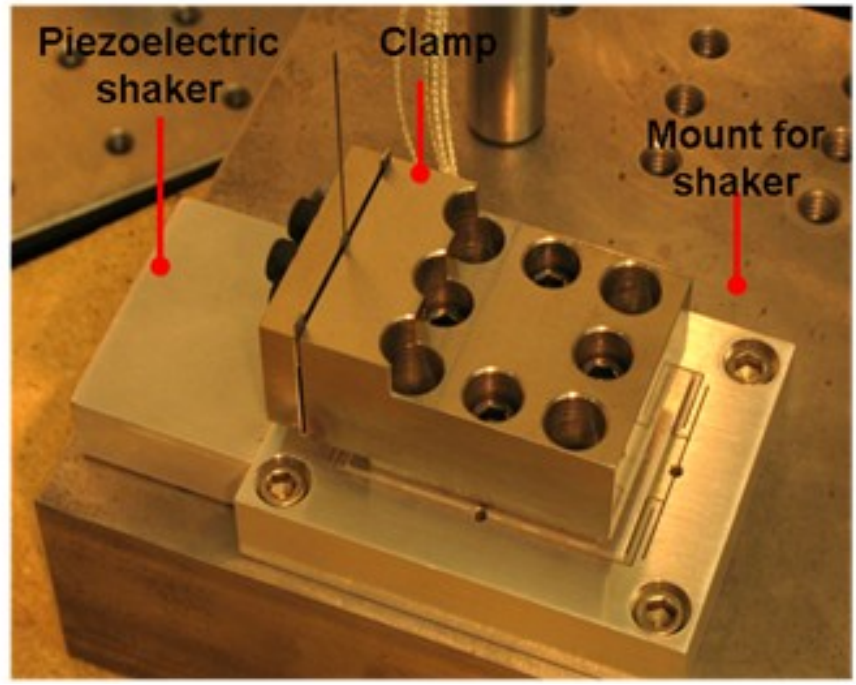

Figure 2.7: a) Exploded view of the subcomponents in the clamp assembly b) photograph of clamp attached to the shaker which in turn is attached to a large stainless steel block that serves as the frame for the system. 


\subsubsection{Selection criteria and operational characteristics of the base excitation system}

The main selection criteria for the base excitation were:

i) A shaker assembly capable of simulating a sinusoidal forcing function delivering an acceleration of $2.5 \mathrm{~ms}^{-2}( \pm 1 \%)$ at a frequency of $60-100 \mathrm{~Hz}$. The required peak to peak displacement range was between $6-18 \mu \mathrm{m}$ with a precision of $\pm 100 \mathrm{~nm}$. The minimum force capability of the shaker assembly was $2 \mathrm{~N}$.

ii) The vibration assembly had to have a relatively high first resonance $>750 \mathrm{~Hz}$ and integrated control to reduce power at resonance to prevent damage

iii) An integrated feedback loop was required to control and maintain output vibration characteristics.

iv) The shaker required a hole pattern and specimen mounting area of at least $25 \times 25 \mathrm{~mm}$ and that was capable of withstanding repeated replacement of test specimens.

v) The shaker had to be vacuum compatible to $10^{-5} \mathrm{mBar}$ (high vacuum). Therefore, all components must be manufactured with materials with minimal outgassing.

The two main options for vibration shakers were electrostatic actuators and piezoelectric stacks or assemblies. Whereas electrostatic actuators provided the desired characteristics and control, an exhaustive search did not reveal small shaker units with proven vacuum compatibility. However high precision piezoelectric stages used in confocal and atomic force microscope assemblies were well suited to provide the necessary vibration characteristics. After evaluating several options from various suppliers, the Nano-OP 65 (Mad City Labs, Wisconsin, USA) single axis piezo electric stage was selected. A brief outline of the specifications of this system is provided in Table 2.3. The stage contains a built-in capacitative displacement sensor (CDS) with sub-nanometer precision that was incorporated in a feedback control loop built into the power supply. 
Table 2.3: Specification of Nano-OP65 piezoelectric stage used for base excitation.

\begin{tabular}{ll}
\hline Operating parameter & Operating range \\
\hline Range of motion (Nano-OP65) & $0-65 \mu \mathrm{m}(0-10 \mathrm{~V})$ \\
Resolution of capacitative displacement sensor & $0.13 \mathrm{~nm}$ \\
Resonant Frequency (unloaded) & $4 \mathrm{kHz} \pm 20 \%$ \\
Stiffness & $3.0 \mathrm{~N} / \mu \mathrm{m} \pm 20 \%$ \\
Recommended max. load (horizontal) & $1.0 \mathrm{~kg}$ \\
Recommended max. load (vertical) & $0.5 \mathrm{~kg}$ \\
Body Material & Aluminum \\
\hline
\end{tabular}

The stage is controlled by a $150 \mathrm{~V}$ amplifier (Nanodrive85) with the option of an analog 0-10 V driving signal input or USB interface with Labview programmable control parameters. The stage has a maximum displacement of $65 \mu \mathrm{m}$ with less than $1 \%$ deviation in displacement control up to $5 \mathrm{~ms}^{-2}$. This means that the full range $0-10 \mathrm{~V}$ corresponds to displacements over the range of $0-65 \mu \mathrm{m}$. The translation of the main stage is in the plane of the body of the device, as shown in Figure 2.7(a), and has a maximum load of $500 \mathrm{~g}$, well exceeding the requirements for our specimens. However, in order to maintain a high value for the first resonance frequency of the shaker, the mass of the clamp was restricted to $175 \mathrm{~g}$. The shaker is itself anchored to a larger stainless steel block for support (Figure 2.7(b)).

\section{Characterization of the dynamic characteristics of the shaker with the clamp}

Understanding the dynamic characteristics of an excitation system is vital for accurate and correct modal analysis (and subsequently, extraction of damping parameters). This is particularly critical for forced excitation studies, where the motion of the shaker is essential for an accurate frequency response function [Ewi00]. Knowing the displacement of the shaker is less critical for free decay measurements in low damped structures where there is no forced excitation beyond the initial driving impulse. All the measurements in this thesis fall into 
this latter category of lightly damped systems. However, the frequency response of the shaker is essential from an operational standpoint because operating the shaker at an internal resonance could cause irreparable damage to it.

To determine the frequency response, the shaker was subject to a sinusoidal voltage signal with constant amplitude of $1 \mathrm{~V}$. The signal was swept over a frequency range from $10 \mathrm{~Hz}$ to $10 \mathrm{kHz}$. The corresponding displacement of the shaker was measured with the built in CDS. All measurements were carried out under vacuum to simulate realistic test conditions. Both the input signal and the output from the CDS were recorded simultaneously during the frequency sweep with a data acquisition unit at a sampling rate of $100 \mathrm{kHz}$. The gain of the shaker over frequency was then obtained by dividing the power spectrum of the CDS signal $\left(G(f)_{\text {shaker }}\right)$ with the input signal $\left(G(f)_{\text {input }}\right)$.

From the results in Figure 2.8, we see that the shaker shows a 1:1 gain up to $\sim 60$ $\mathrm{Hz}$ after which the gain drops by over two orders of magnitude up to $1 \mathrm{kHz}$. Following that are several resonance peaks occurring at $\sim 940 \mathrm{~Hz}, 1265 \mathrm{~Hz}, 1340$ $\mathrm{Hz}$ and $2120 \mathrm{~Hz}$. Therefore, these resonance points were avoided for any damping measurements and during frequency sweeps to prevent the shaker from being damaged. Beyond $2700 \mathrm{~Hz}$, there are no measureable resonance peaks up to $70 \mathrm{kHz}$. Measurements were also carried out without the added mass of the clamp. It was found the additional mass of the clamp lowers the internal resonance frequencies but the magnitudes of the peaks are still small and remain relatively unchanged. 
(a)
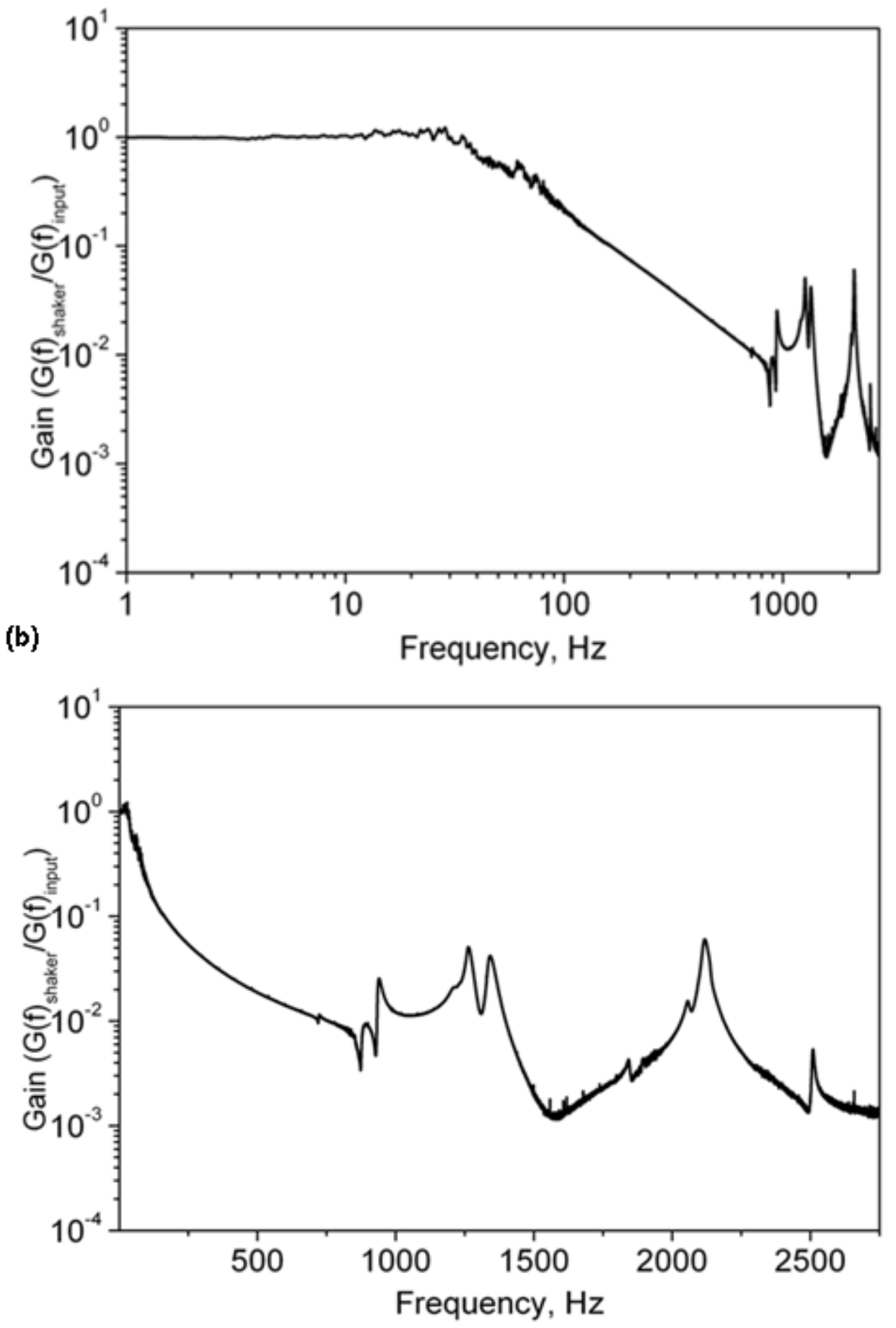

Figure 2.8: The gain of shaker as a function of frequency a) log-log plot clearly showing the response up to $600 \mathrm{~Hz} \mathrm{~b}$ ) standard plot to highlight the resonance peaks above $600 \mathrm{~Hz}$. 


\subsubsection{Selection and design of the vacuum system}

The piezoelectric shaker, with clamp and beam attached, was designed to be placed in the vacuum chamber in an upright position as shown in Figure 2.9(a). The vacuum chamber was commercially purchased (LACO Technologies, USA) and consists of a stainless steel chamber ( 8 inch diameter and 10 inch tall) with six access ports for the pump, gas control and electrical feed throughs. The chamber is connected to a turbo-molecular pump (Adixen ATP80 turbo pump, Alcatel Vacuum, Canada Inc) and backed by a low vacuum roughing pump (Alcatel Pascal 2005SD, Alcatel Vacuum, Canada Inc) with a manually operated valve system. All pump connections were selected to be flexible stainless steel bellows so as to attenuate vibrations from the pumps. All connections are KF kwik-flange connectors with wing-nuts for simple assembly and maintenance of all components. The pressure in the chamber is monitored by a Pirani gauge with a digital readout.
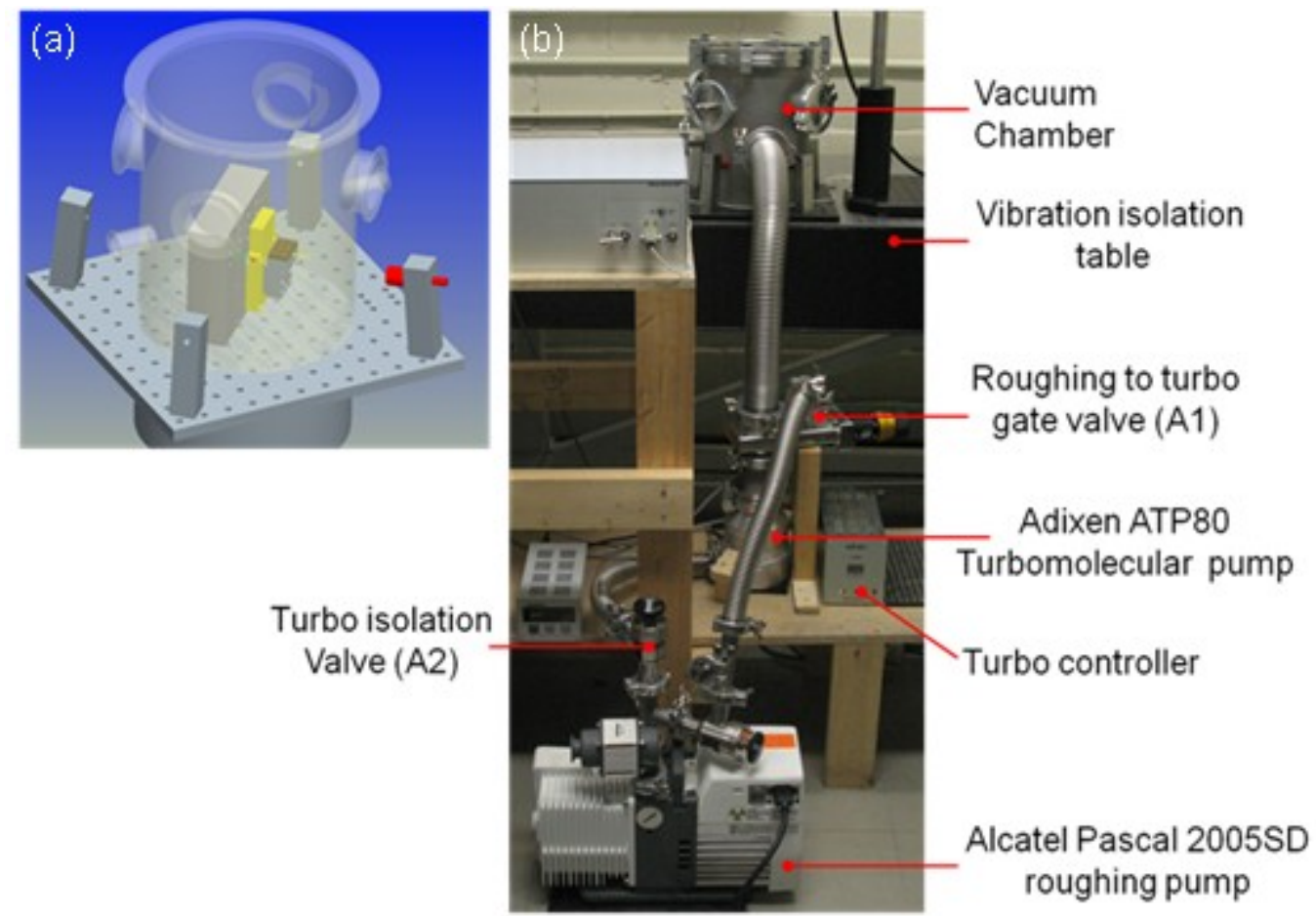

Figure 2.9: a) Design schematic showing shaker, clamp and beam when loaded into chamber b) Photograph of the high-vacuum assembly designed for measurements. 
When pumping down, the roughing pump is used to pump down to pressures less than $<1 \mathrm{mBar}$ before introducing the turbo-molecular pump into the cycle. The system then takes approximately 15 minutes to pump down to a pressure of $1 \times 10^{-4} \mathrm{mBar}$, which is sufficiently low to make viscous damping negligible in our specimens, as discussed in section 2.4. The system can be rapidly vented with the aid of a manual bleed valve, thereby greatly reducing the time between measurements.

In an effort to isolate the chamber and measurement setup from ambient vibrations, the entire assembly was securely fastened to a large pneumatic vibration isolation table. The efficacy of the table in damping vibrations was assessed with an accelerometer (tri-axial ICP Model 356A32, PCB Piezotronics, Miniature accelerometers). Several measurements of the vertical acceleration were taken at various locations on the table. From the power spectrum results in Figure 2.10 , we see that the table has a number of pronounced vibration peaks between 30 and $40 \mathrm{~Hz}$.

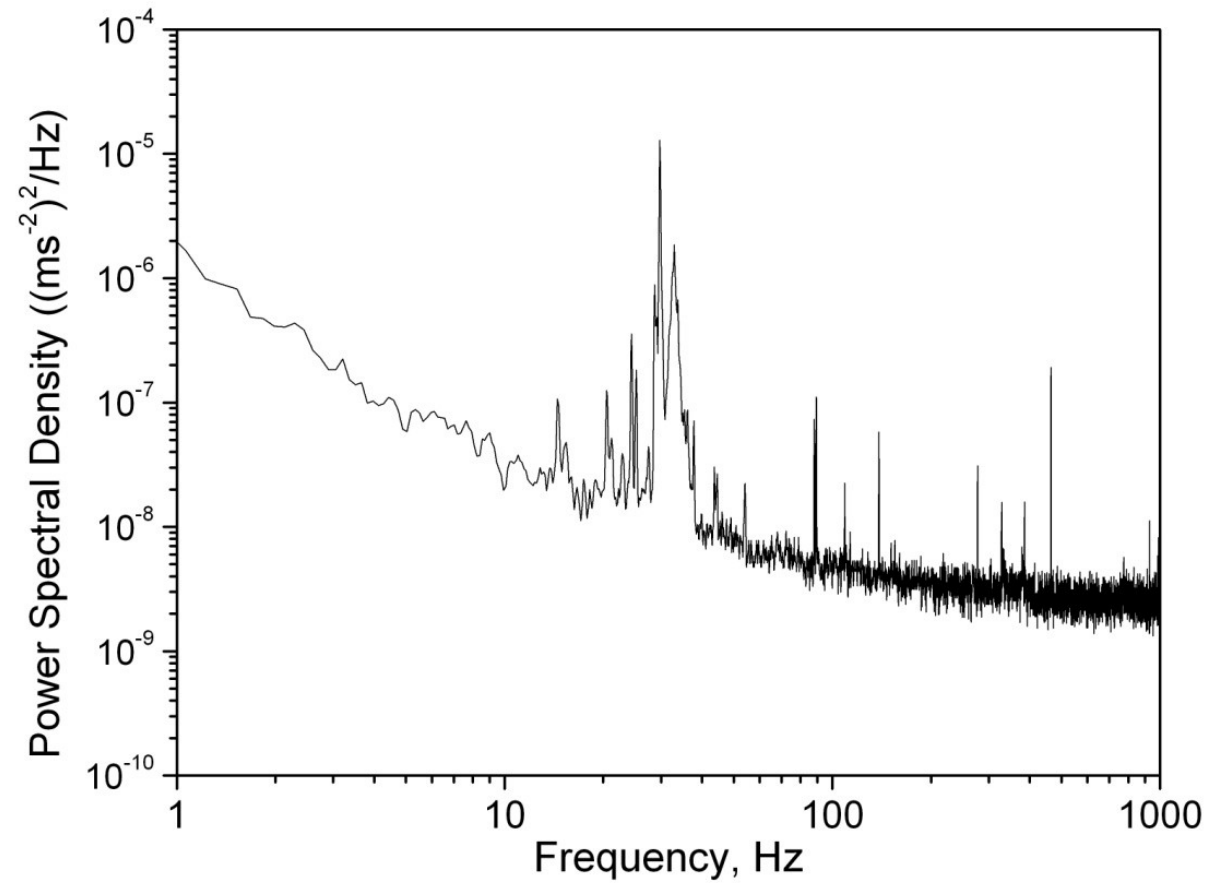

Figure 2.10: Power spectrum of vertical acceleration signal from an accelerometer mounted on the pneumatic vibration isolation table. 
Although the acceleration is low, it can have an impact on measurements of damping in the vicinity of 30-40 Hz. It is not immediately clear why the table responds in this manner. Two possible reasons are:

i) The table is not completely isolated from ground vibrations as it is connected to the pump (and therefore, the ground) via the vacuum pipes on the chamber. However, the vibration characteristics were the same regardless of whether the pump was on or off.

ii) The load on the table is insufficient: The legs are designed to isolate vibrations above $10 \mathrm{~Hz}$ for a full load of approximately $300 \mathrm{lbs} / \mathrm{leg}$. However, the table top and vacuum setup is significantly lighter than this and shifts the resonance to higher frequencies.

\subsubsection{Measuring the displacement/velocity of the beam}

The dynamics of the beams can be measured with two alternative approaches. The first is using a fiber optic displacement probe sensor (Philtec D20 optical probe, Philtec Inc, MD, USA) that is fed through into the chamber. This probe consists of several optical fibers $(25-50 \mu \mathrm{m}$ in diameter) that are sandwiched together to form the measurement tip. A set of these fibers is selected to emit light and the remainder sense the intensity of reflected light from the target surface [Ran02].

To measure the displacement, the probe is pointed at and brought into close proximity with the surface. At a calibrated standoff distance, small displacements of the surface are linearly related to a change in the reflected intensity of light. This change in intensity is obtained as a scaled voltage output from the detector. The specifications of the fiber optic probe are provided in Table 2.4. Crucially, the probe has a very small standoff distance $(300 \mu \mathrm{m})$ and, therefore, the calibration and setup must be carried out outside the chamber as shown in Figure 2.11. The entire assembly is then loaded into the chamber and no further adjustments can be made to the signal under vacuum. 


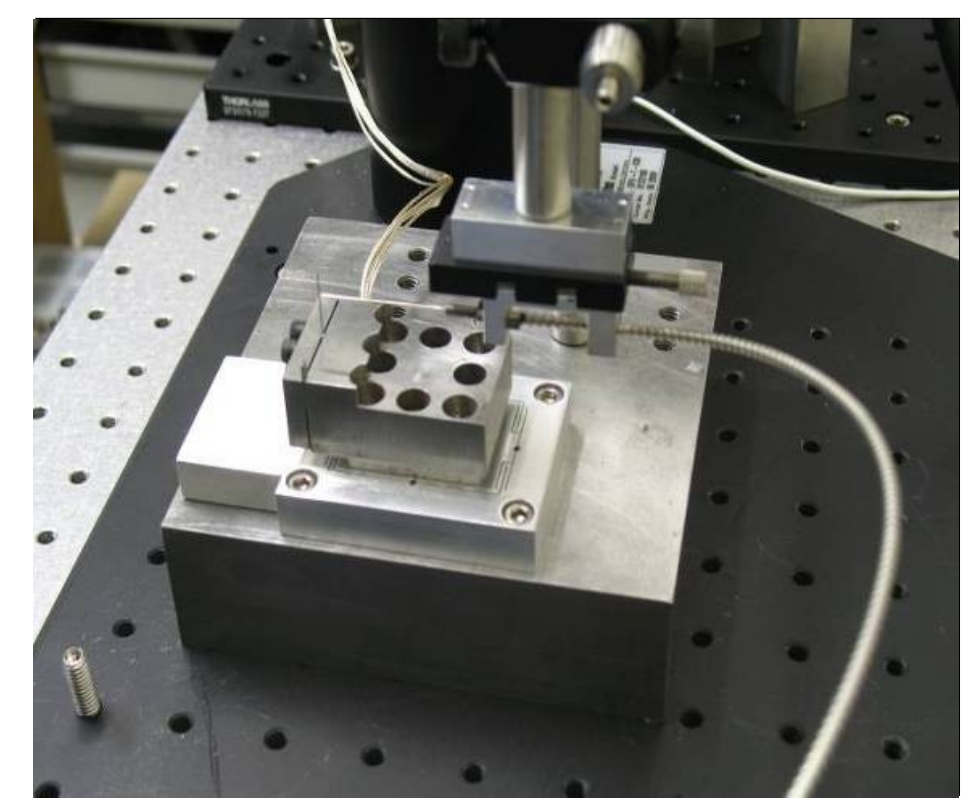

Figure 2.11: Photograph of shaker, clamp and beam setup with fiber optic probe positioned to measure the displacement of the beam.

The second, more sensitive and more convenient, method of measuring the dynamics of the beam is using a laser Doppler Vibrometer (LDV, Polytec GmbH, Germany). The LDV works on the principle of shining a laser of known wavelength on the vibrating surface and measuring the frequency shift of backscattered light. This frequency shift, known as the Doppler effect, is proportional to the velocity at a given wavelength [Ran02] and can be used to determine the velocity of the surface. The entire LDV setup consists of a sensor head equipped with a $633 \mathrm{~nm}$ neon laser and a built in camera (OFV534 fiber coupled vibrometer sensor head) and a decoder to process the voltage output from the laser head (OFV5000 VD09 decoder). Figure 2.12 shows an image of the measurement setup with the laser head positioned outside the vacuum chamber. The standoff distance is approximately $30 \mathrm{~cm}$ for this system.

Table 2.4 shows a comparison of the optical fiber sensor and LDV. The latter has a higher resolution, a smaller spot size (can be used on smaller structures) and a much larger standoff distance. Initial measurements were made using both the optical fiber and the LDV and led to identical results. 
Table 2.4: Capabilities of the fiberoptic displacement sensor and the laser Doppler Vibrometer.

\begin{tabular}{lcc}
\hline & $\begin{array}{c}\text { D20 Fiber optic } \\
\text { Sensor }\end{array}$ & $\begin{array}{c}\text { LDV with VD09 } \\
\text { Decoder }\end{array}$ \\
\hline Resolution & $0.25 \mu \mathrm{m}$ & $0.02 \mu \mathrm{ms}^{-1} \mathrm{~Hz}^{-1 / 2}$ \\
Linear range & $200 \mu \mathrm{m}$ & $0.05 \mathrm{~ms}^{-1}$ \\
Standoff distance & $300 \mu \mathrm{m}$ & $30 \mathrm{~cm}$ \\
Measurement spot size & $500 \mu \mathrm{m}$ & $40 \mu \mathrm{m}$ \\
Frequency range & $0-500 \mathrm{kHz}$ & $0-100 \mathrm{kHz}$ \\
\hline
\end{tabular}

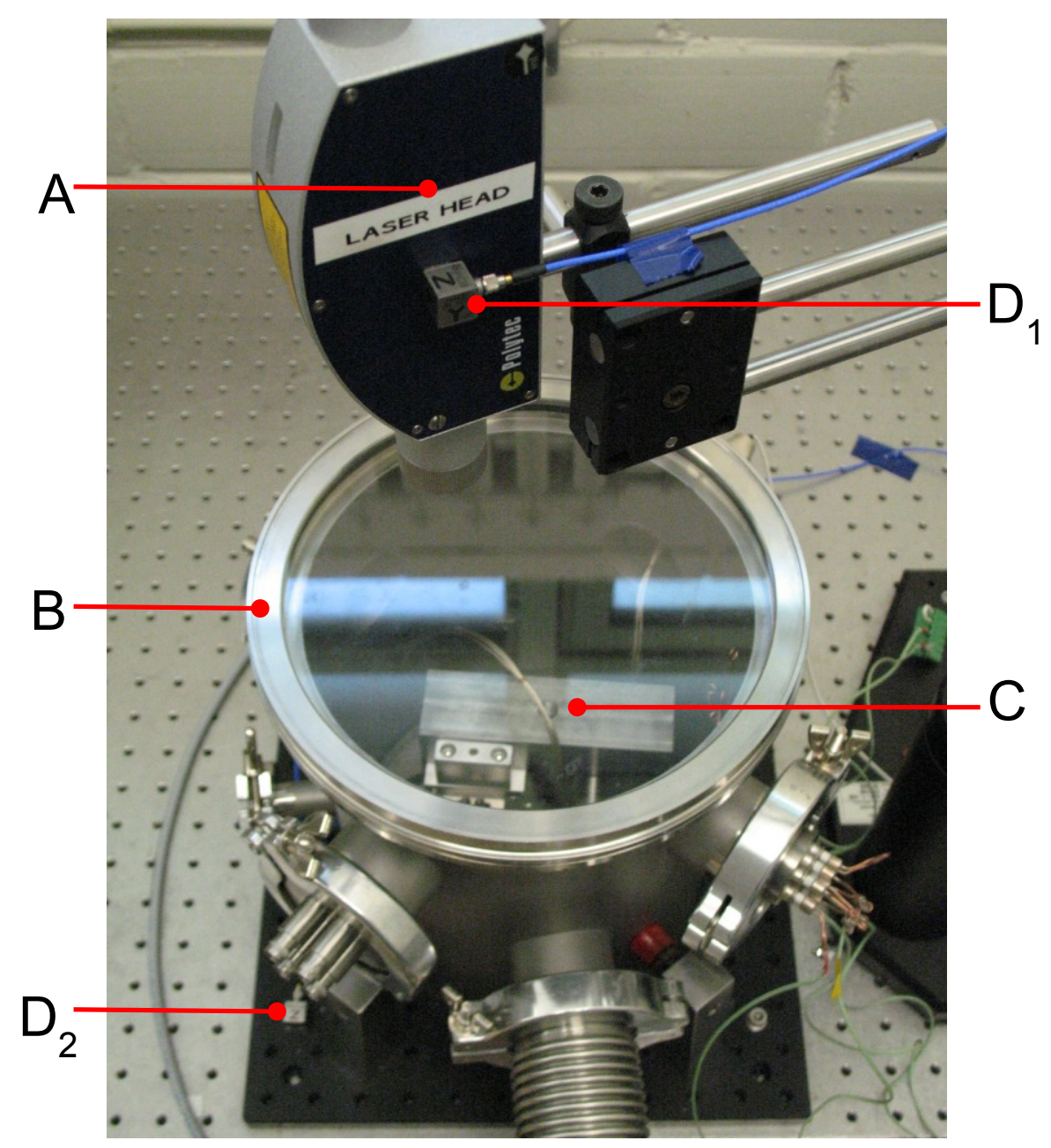

Figure 2.12: Photograph of the test apparatus used for measuring the dynamics of microcantilevers. (A) laser Doppler vibrometer, (B) vacuum chamber and (C) mount for the shaker, clamp and cantilever. Accelerometers on LDV $\left(\mathrm{D}_{1}\right)$ and on the table $\left(D_{2}\right)$ were used to characterize the dynamics of the laser head and table respectively. 


\section{Characterization and calibration of the signal from the LDV}

As shown in Figure 2.12, the LDV is positioned on a rigid, cantilevered slide and is suspended over the vacuum chamber. Any vertical vibrations of the LDV will affect the measured dynamics of the silicon beams. Therefore, before beginning any measurements, the vibration characteristics of the LDV were first assessed. The vertical acceleration of the LDV was measured with a triaxial accelerometer similar to the one used to measure the table vibrations. Figure 2.13(a) shows the motion of the LDV alone and shows distinct acceleration peaks between 20 and $40 \mathrm{~Hz}$ with a maximum acceleration of $0.04 \mathrm{~ms}^{-2}$ and a small peak at $\sim 400 \mathrm{~Hz}$, which is not visible in the vibration of the table.

To determine the frequency response of the LDV setup to the vibrations of the table, the signals from the two accelerometers (Model 356A32, PCB Piezotronics, Miniature accelerometers) positioned as shown in Figure 2.12 were recorded simultaneously. The magnitude of the frequency response function $(|H(f)|)$ of the LDV to the table was then calculated as [Ben00]

$$
|H(f)|^{2}=\frac{G(f)_{L D V}}{G(f)_{\text {Table }}}
$$

where $G(f)_{L D V}$ and $G(f)_{\text {Table }}$ are the power spectrum of the vertical acceleration of the LDV and the table, respectively. Figure 2.13(b) is the magnitude of the frequency response and provides a measure of the effect of the table on the observed oscillation in the LDV signal.

Taken together, the results shown in Figure 2.13 establish the lower frequency limit on measurements of damping. In this thesis, no measurements are carried out below $70 \mathrm{~Hz}$. In addition, for measurements above $100 \mathrm{~Hz}$, a high pass filter built into the LDV decoder was utilized to eliminate low frequency noise. Measurements of damping with and without the filter showed no difference other than in the signa- to-noise ratio. 

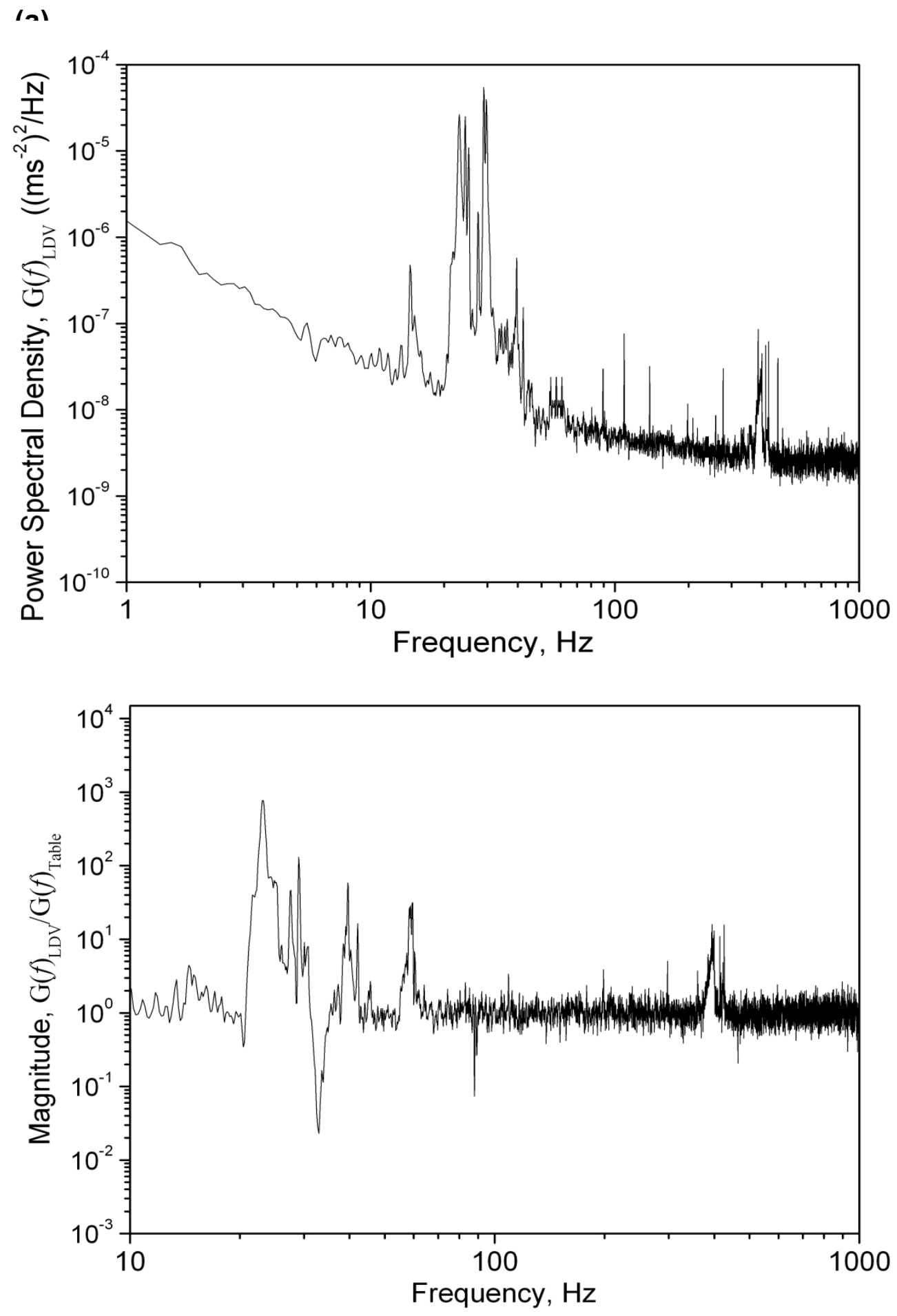

Figure 2.13: a) power spectrum of vertical acceleration of the LDV b) magnitude of the frequency response of the acceleration of the LDV to the table. 
Next, the LDV was checked for accuracy and, more critically, for linearity using the piezoelectric shaker base (with mating plate Figure 2.7(b)) as the reference. For both tests, the laser spot was positioned on the mating plate shown in Figure 2.7 and the shaker oscillated.

To check for accuracy, the shaker was oscillated at various frequencies (20-120 $\mathrm{Hz})$ and at various oscillation amplitudes $(0.2-1 \mathrm{~V})$. During the oscillations, the velocity signal from the LDV was converted to displacement (by dividing by the frequency) and then compared with the displacement signal from the CDS. A representative measurement of the two signals at $20 \mathrm{~Hz}$ is shown in Figure 2.14. Over the range of frequencies and amplitudes, measurements with the LDV followed the form of the displacement sensor accurately (with the appropriate $90^{\circ}$ phase shift), but were consistently higher than the values from the CDS at frequencies below $40 \mathrm{~Hz}$. This difference is due to the superposition of the low frequency vibrations of the LDV head on the shaker signal and is not seen at frequencies above $70 \mathrm{~Hz}$

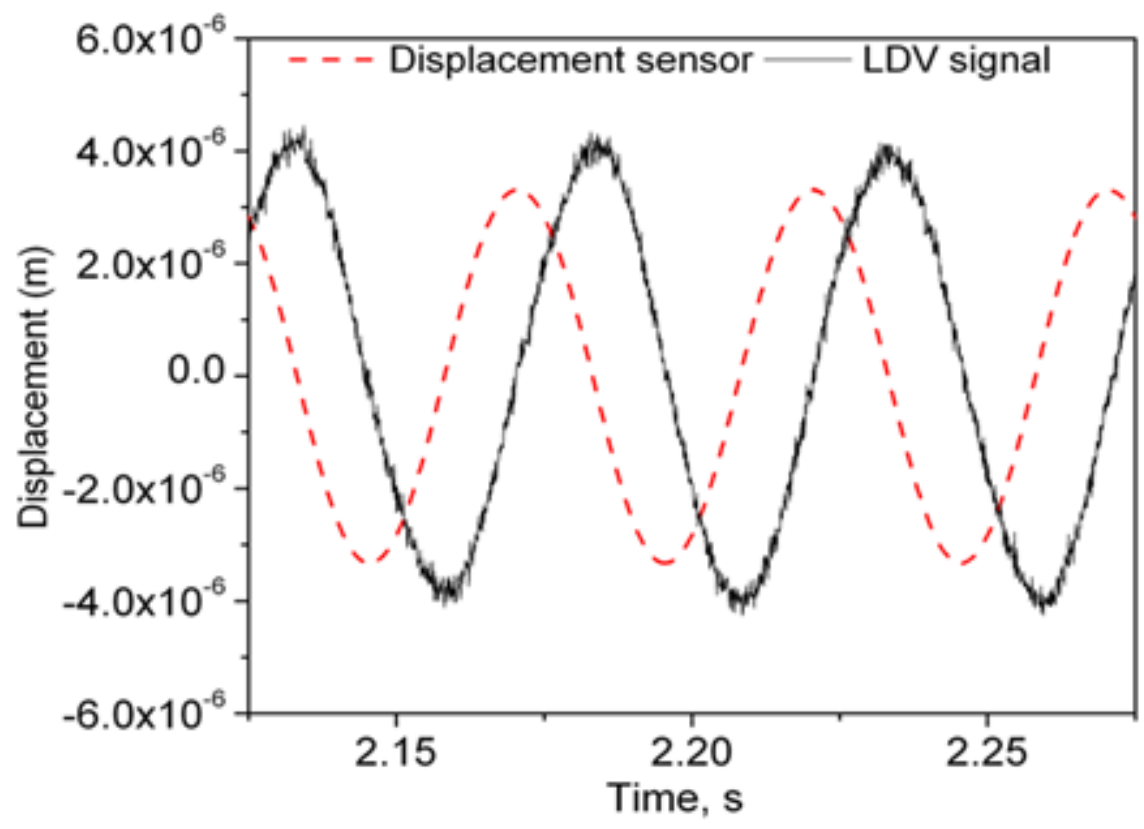

Figure 2.14: Representative signals of the displacement of the shaker base measured with the built in CDS and the LDV at $20 \mathrm{~Hz}$. 
To check for linearity, the shaker was oscillated at a single frequency $(110 \mathrm{~Hz})$ and the oscillation amplitude changed by increasing the input voltage $(0.5-3 \mathrm{~V})$. The root mean squared displacement was then calculated from the output of the LDV. Figure 2.15 shows the signal from the LDV signal as a function of the applied voltage demonstrating the linearity and therefore validating the use of this tool to measure damping.

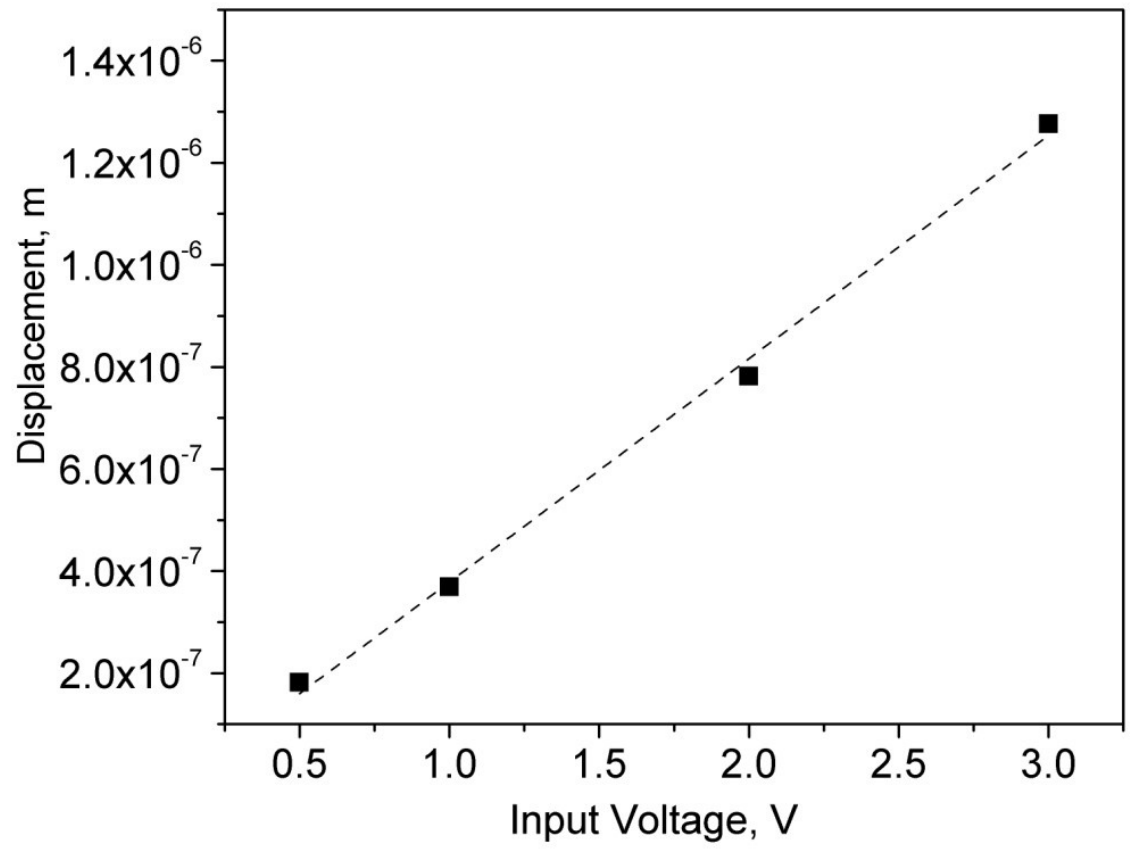

Figure 2.15: Plot of displacement of the shaker as a function of applied voltage as measured by the LDV. The dashed line is the linear least-squares best fit line to data with $\mathrm{R}^{2}=0.99$.

This concludes the design aspects of the setup and the following sections describe the approach to measuring damping in the single-crystal silicon beams using the logarithmic decrement approach. 


\subsection{Approach to measuring damping}

There are numerous experimental approaches to measure damping in structures and these approaches can be divided into two main categories: i) Time domain techniques where the system is subjected to initial conditions and the damping is extracted from the free response (logarithmic decrement and step response method), and ii) frequency domain techniques that rely on the steady state response of the system to a continuous harmonic forcing function (magnificationfactor and bandwidth method). Detailed reviews and analysis of the various methods including proofs of equivalence for the various measures of damping can be found in references [Ber73, Gra92, Sil00, Ewi00]. For ease of implementation in lightly damped structures, the main approach utilized in this thesis is the logarithmic decrement.

\subsubsection{Extraction of damping using the logarithmic decrement ( $\delta$ )}

The log decrement method is a widely used time response method and is based on measuring the free response of an underdamped single degree of freedom system [Now72, Gra92, Sil00, Ewi00]. The typical procedure is to first excite the structure near one of its resonance frequencies using a time harmonic force. The excitation is then terminated and the corresponding decay signal measured. For low damped structures, the decay is usually observed to be exponential and the natural logarithm of the ratio of two consecutive amplitudes of oscillation is a measure of the dissipation. This measure of dissipation is termed the logarithmic decrement.

To illustrate the method of extracting the logarithmic decrement and its equivalence to material damping, let us examine the free decay of an underdamped cantilevered beam. The formulation that follows is based on the approach outlined by Banks and Inman [Ban91]. 


\section{Free decay of a damped cantilevered Euler-Bernoulli beam}

Consider a thin slender cantilevered beam of uniform rectangular cross section attached to a large base as shown schematically in Figure 2.1. The monolithic beam, henceforth referred to as the substrate, has a length $L$, a rectangular cross section of thickness $h_{s}$ and width $w$, and has a density $\rho$. We assume that the viscous, clamping and support losses have been minimized such that material damping dominates the damping in the beam. Here we assume a Kelvin-Voigt (KV) form of material damping which is a simple and widely used model of anelastic material behaviour [Now72, Ber73, Gra92]. Also known as strain-rate damping [Ban91], KV damping is analogous to a dissipative viscous dashpot used in a single degree of freedom (SDOF) spring-mass-dashpot system [Sil00].

The dynamics of the structure can be modelled as an Euler-Bernoulli beam executing flexural vibrations with an equation of motion of the form

$$
\rho A \frac{\partial^{2} u(x, t)}{\partial t^{2}}+\frac{\partial^{2}}{\partial x^{2}}\left(E I \frac{\partial^{2} u(x, t)}{\partial x^{2}}+C_{d} I \frac{\partial}{\partial t}\left(\frac{\partial^{2} u(x, t)}{\partial x^{2}}\right)\right)=0
$$

where $C_{d}$ is the strain-rate (KV) damping constant, $I$ is the area moment of inertia, $E$ is the Young's modulus of the material used in the beam.

The solution to Equation (2.13) can be written as

$$
u_{n}(x, t)=y_{n}(t) \phi_{n}(x)
$$

where the $\phi_{n}(x)$ and $y(t)$ are the $n^{\text {th }}$ eigen mode shape and the time response of the beam respectively. Substituting Equation (2.14) into Equation (2.13) and separating variables, we obtain two second order linear differential equations:

$$
\frac{d^{4} \phi_{n}(x)}{d x^{4}}+\alpha_{n}^{4} \phi_{n}(x)=0
$$




$$
\frac{d^{2} y_{n}(t)}{d t^{2}}+\alpha_{n}^{4}\left(\frac{C_{d} I}{\rho A} \frac{d y_{n}(t)}{d t}+\frac{E I}{\rho A} y_{n}(x)\right)=0
$$

From Equation (2.15a), for a cantilevered beam with one end clamped and the other free to oscillate, the $n^{\text {th }}$ eigen mode shape is given by [Sil00]

$$
\phi_{n}(\xi)=A_{0}\left(\sin \left(\alpha_{n} \xi\right)-\sinh \left(\alpha_{n} \xi\right)+\left(\frac{\sin \left(\alpha_{n}\right)+\sinh \left(\alpha_{n}\right)}{\cos \left(\alpha_{n}\right)+\cosh \left(\alpha_{n}\right)}\right)\left(\cosh \left(\alpha_{n} \xi\right)-\cos \left(\alpha_{n} \xi\right)\right)\right),
$$

where $\mathrm{A}_{0}$ is the maximum amplitude of vibration of the tip and $\xi=x / L$ is the normalized length. Turning to Equation (2.15b), we see that it is of the form of a single degree of freedom system with viscous damping and can be represented as [Ban91]

$$
\frac{d^{2} y_{n}(t)}{d t^{2}}+2 \zeta_{n} \omega_{n} \frac{d y_{n}(t)}{d t}+\omega_{n}^{2} y_{n}(t)=0
$$

Comparing the coefficients, we see that

$$
\omega_{n}^{2}=\alpha_{n}^{4} \frac{E I}{\rho A}, \text { and } 2 \zeta_{n} \omega_{n}=\alpha_{n}^{4} \frac{C_{d} I}{\rho A}
$$

The free response of this system in the time domain is

$$
y_{n}(t)=e^{-\zeta_{n} \omega_{n} t}\left(K_{1, n} \cos \left(\omega_{d n} t\right)+K_{2, n} \sin \left(\omega_{d n} t\right)\right),
$$

where $\omega_{d n}=\omega_{n} \sqrt{1-\zeta^{2}}$ is the damped natural frequency of the system and constants $K_{1, n}$ and $K_{2, n}$ are determined from the initial conditions of the motion of the beam.

Therefore the free decay motion of a cantilevered beam in a single mode is given by substituting Equation (2.19) into Equation (2.14) to yield

$$
u_{n}(\xi, t)=\phi_{n}(\xi) e^{-\zeta_{n} \omega_{n} t}\left(K_{1, n} \cos \left(\omega_{d n} t\right)+K_{2, n} \sin \left(\omega_{d n} t\right)\right)
$$




\subsubsection{Equivalence of logarithmic decrement to the damping factor}

Let us now extract the logarithmic decrement from the free decay of this cantilever in the first mode $(n=1)$. The basic approach measures the ratio of the amplitude response at a time $t=t_{i}$ to the amplitude after $r$ number of cycles, where $t=t_{i}+2 \pi r / \omega_{d 1}$. Using Equation (2.20), the ratio of amplitudes is given by

$$
\frac{u_{1}\left(1, t_{i}+2 \pi r / \omega_{d 1}\right)}{u_{1}\left(1, t_{i}\right)}=e^{\left(-\zeta_{n} \frac{\omega_{1}}{\omega_{d 1}} 2 \pi r\right)} .
$$

Therefore, the logarithmic decrement, $\delta_{1}$, per unit cycle is

$$
\delta_{1}=\frac{1}{r} \ln \left(\frac{u_{1}\left(1, t_{i}+2 \pi r / \omega_{d 1}\right)}{u_{1}\left(1, t_{i}\right)}\right)=\zeta_{1} \frac{\omega_{1}}{\omega_{d 1}} 2 \pi=\frac{2 \pi \zeta_{1}}{\sqrt{1-\zeta_{1}^{2}}} .
$$

For low values of damping ratios $\left(\zeta_{1}<0.01\right)$, Equation (2.22) can be simplified to

$$
\delta_{1} \approx 2 \pi \zeta_{1} .
$$

and can be related to the strain-rate dependent property of the material through Equation (2.18).

In a similar manner, the logarithmic decrement has been shown to be a useful experimental measure of damping for other models of material damping such as the complex spring [Now72, Ber73, Gra92] and the standard anelastic solid [Zen47].

The logarithmic decrement approach is relatively simple to implement. By simply picking peak amplitudes per cycle and using Equation (2.22), we can obtain an estimate of the damping. Alternately, the damping can be obtained from the slope of a linear least-squares fit to the logarithm of the peak amplitudes. However, in both cases the challenge is to isolate the peaks. There are no standard approaches to peak selection and the presence of noise can make this approach inaccurate for lightly damped systems. Instead, using the Hilbert transform offers a more computationally robust approach to calculate the damping, as described in the next section. 


\subsubsection{Hilbert transform approach to obtain damping factor}

The discussion presented in this section draws upon the work of Feldman [Fe106] and Smith and Werely [Smi96]. The Hilbert transform is an integral transform that can be utilized to examine the instantaneous frequency, phase and amplitude of the vibration signal. In free decay measurements where we are primarily interested in the decay envelope to extract the damping, the Hilbert transform provides an elegant approach to determine the instantaneous damping and frequency. To illustrate the action of the Hilbert transform, let us examine a simple, single frequency, time harmonic signal

$$
x(t)=A \cos \left(\omega_{n} t\right)
$$

where $A$ is the amplitude and $\omega_{n}$ is the frequency of the signal. The Hilbert transform operation is a filter in which the amplitude of the spectral signal remains unchanged but the phases are shifted by $-\pi / 2$ for positive frequencies [Fe106]. Therefore, the Hilbert transform of $x(t)$ is

$$
\widetilde{x}(t)=\mathrm{H}(x(t))=A \cos \left(\omega_{n} t-\frac{\pi}{2}\right)=A \sin \left(\omega_{n} t\right)
$$

Of most relevance here is that the Hilbert transform can also be interpreted as the imaginary part of an analytic signal. In this context, the term 'analytic' refers to a complex function of a complex number [Fel06]. Taking the function in Equation (2.25) and its Hilbert transform, we can define an analytic function:

$$
z(t)=x(t)+\tilde{i x}(t)
$$

This signal can also be represented in complex form

$$
z(t)=Z(t) e^{i \theta(t)}
$$

where $Z(t)$ is the envelope of the function $x(t)$ and $\theta(t)$ is called the instantaneous phase of the signal $x(t)$ [Ben00]. In terms of $x(t)$ and $\widetilde{x}(t)$ 


$$
\begin{gathered}
Z(t)=\sqrt{x(t)^{2}++\widetilde{x}(t)^{2}}=A, \\
\theta(t)=\tan ^{-1}\left(\frac{\tilde{x}(t)}{x(t)}\right)=\omega_{n} t
\end{gathered}
$$

In a similar fashion, we can apply this analysis to the free decay signal of a cantilevered beam and obtain the envelope of the exponential decay. Let us take the free decay of the form in Equation (2.20). The Hilbert transform of $u_{n}(\xi, t)$ is

$$
\tilde{u}_{n}(\xi, t)=\phi_{n}(\xi) e^{-\zeta_{n} \omega_{n} t}\left(K_{1, n} \sin \left(\omega_{d n} t\right)-K_{2, n} \cos \left(\omega_{d n} t\right)\right)
$$

We can also define an analytic signal of the decay as

$$
z_{n}(\xi, t)=u_{n}(\xi, t)+i \tilde{u}_{n}(\xi, t)=Z_{n}(\xi, t) e^{i \theta(t)}
$$

The magnitude of the analytic signal provides the envelope of the decay signal as

$$
Z_{n}(\xi, t)=\sqrt{u_{n}(\xi, t)^{2}+\widetilde{u}_{n}(\xi, t)^{2}}=\phi(\xi) K_{0} e^{-\zeta_{n} \omega_{n} t}
$$

where $K_{0}=\sqrt{K_{1, n}^{2}+K_{2, n}^{2}}$ and is determined by the initial conditions. Taking measurements at the end of the tip $(\xi=x / L=1)$ and for low values of damping $\left(\zeta_{n}<0.01\right)$, we can approximate the envelope of the free decay in terms of the logarithmic decrement, $\delta_{n}$, as

$$
Z_{n}(1, t)=\phi(1) K_{0} e^{-\left(\zeta_{n} \omega_{n} t\right)} \approx \phi(1) K_{0} e^{-\left(\frac{\delta_{n}}{2 \pi} \omega_{n} t\right)}
$$

The damping can be obtained by fitting a line to the logarithm of the envelope signal. The slope of this line is $\frac{\delta_{n}}{2 \pi} \omega_{n} t$. For lightly damped structures, $\omega_{n} \approx \omega_{d n}$ and can be obtained from the power spectrum of the decay signal (it should be noted that the time $t$ should be appropriately scaled with the sampling rate). Figure 2.16 shows a representative free decay signal and the corresponding envelope derived from Equation (2.31). 


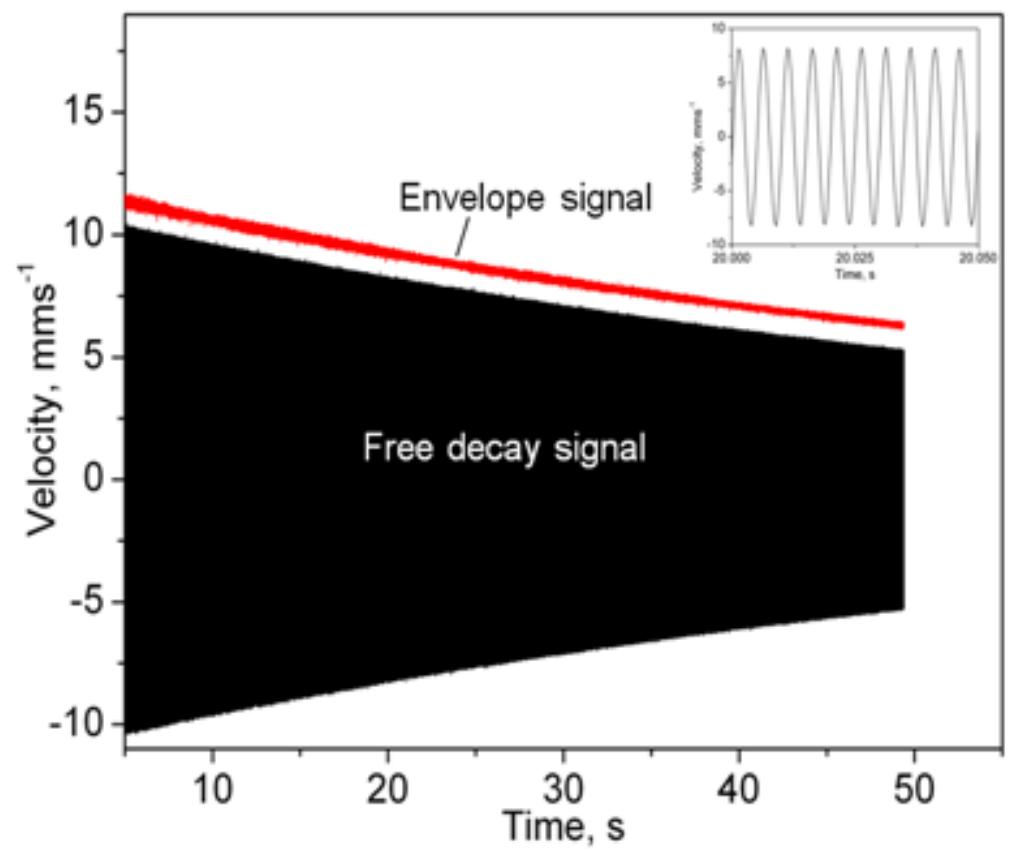

Figure 2.16: Plot of the free decay signal of a silicon cantilever and its envelope as calculated from as described in Equation (2.31). The envelope is offset slightly for clarity of presentation and the inset shows the signal for a few cycles of vibration.

For all measurements in this thesis, the Hilbert transform was utilized to extract the logarithmic decrement. The next section describes the standard procedures followed to extract the dissipation in the beams for the first mode.

\subsubsection{Procedure to measure damping in the fundamental mode}

Figure 2.17 shows the results of a typical measurement on a graph of the tip velocity as a function of time. To measure the damping in the single-crystal silicon beams, the base of the microcantilever is first excited at a frequency close to the fundamental frequency for flexural vibrations. The low damping in the beams $\left(\delta_{s}<10^{-4}\right)$ means that very little excitation force is required. Therefore, the shaker is oscillated only by few tens of nanometers in amplitude. 


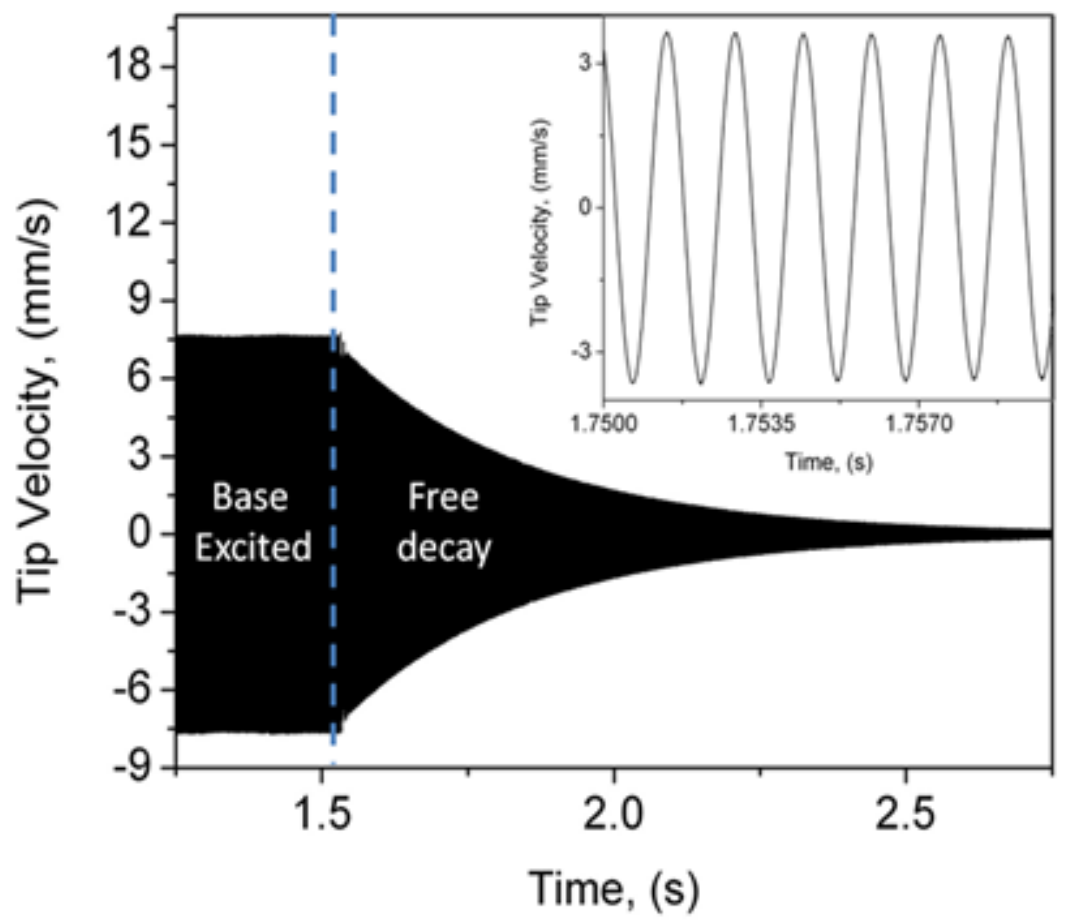

Figure 2.17: Measurement of beam dynamics using laser Doppler vibrometry. The graph shows the tip velocity as a function of time during harmonic excitation and free decay. The inset shows the details of the velocity for a few cycles of vibration.

Subsequently, the base excitation is terminated and the free decay of the beam measured and recorded with a custom program through a data acquisition unit. The sampling frequency is at least ten times greater than the decay frequency to minimize any aliasing effects.

The logarithmic decrement is obtained from the envelope of the free decay using the standard approach and also by using the Hilbert transform as described in section 2.4.1.2. Both methods lead to identical results for the logarithmic decrement of the beam. In addition, the slope of the decay envelope was examined to ensure that the damping was constant over the entire decay of the beam.

During the decay, the signal from the CDS is also recorded. This is done to verify that the decay is not due to coupled body motion between the beam and the clamp. Figure 2.18 shows a representative power spectrum of the cantilever decay signal and the base motion during the decay, clearly demonstrating that there is no measureable interaction and the clamp remains stationary during decay. 

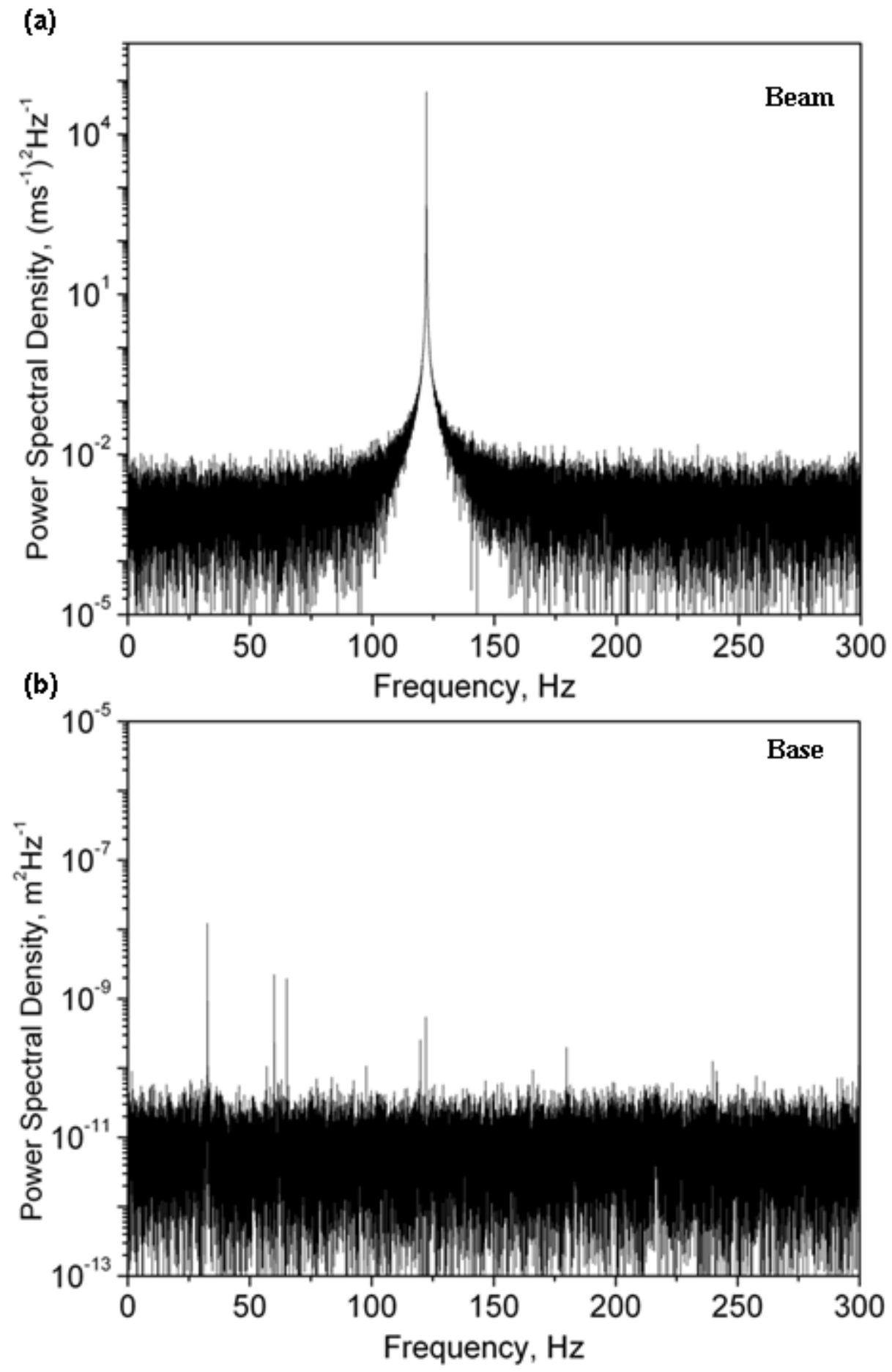

Figure 2.18: Power spectral density of: a) velocity of the tip of a representative silicon cantilever measured with LDV and b) displacement of the piezo shaker base measured with the capacitative sensor during the free decay of the same beam. 
This concludes the section on the design of the experimental setup. The following sections and chapters all utilize the specimens and setup described in section 2.3. and 2.4. We are now ready to move to measurements of damping in single-crystal silicon resonators.

\subsection{Measurement of damping in micromachined single-crystal silicon resonators}

For the first set of studies, seventy different silicon beams were micromachined and characterized. These microcantilevers range from 40 to $131 \mu \mathrm{m}$ in thickness, 0.72 to $1.1 \mathrm{~mm}$ in width, and 0.21 to $3.6 \mathrm{~cm}$ in length. The length-to-thickness aspect ratio of the beams ranges from 58 to 834 , and the fundamental frequency of bending vibrations ranges from $51 \mathrm{~Hz}$ to $10.9 \mathrm{kHz}$. The measured natural frequency for these beams was within $7 \%$ of estimates obtained from a standard analysis of the flexural vibrations of a slender, elastic, Euler-Bernoulli beam.

\section{Effect of vacuum on damping in single-crystal silicon beams}

Figure 2.19 shows a typical measurement of damping in a single-crystal silicon beam as the pressure is reduced in the chamber. The damping in the beam decreases by over 3 orders of magnitude from $10^{-2}$ to $2 \times 10^{-5}$ as the pressure is reduced from atmospheric pressure to $4 \times 10^{-4} \mathrm{mBar}$. Below this pressure viscous damping is not a dominant loss mechanism. All the measurements in this thesis were carried out at a chamber pressure less than $1.5 \times 10^{-4} \mathrm{mBar}$. 


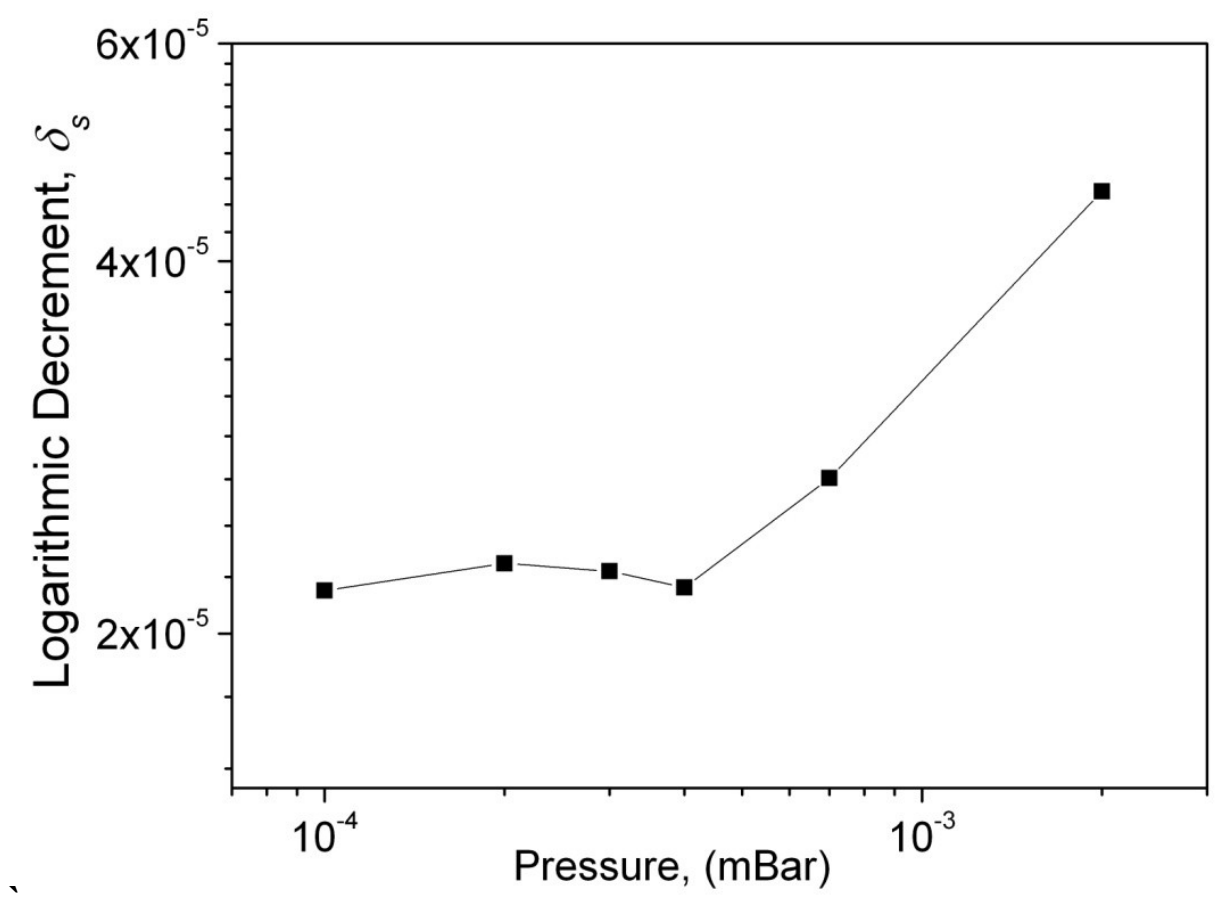

Figure 2.19: Representative pressure dependence of damping (logarithmic decrement) in single-crystal silicon beams as a function of pressure in the vacuum chamber. Under ambient pressure $(\sim 1$ Bar), the logarithmic decrement in the beams is in the order of $10^{-2}$.

\section{Repeatability of measurements}

To assess the repeatability of the measurements, eight beams were selected and measured at least three times. Each measurement included a complete re-mount, pump down, and three separate measurements of natural frequency and damping. Across all these 72 measurements, the variation in the natural frequency and logarithmic decrement was less than $0.1 \%$ and $7 \%$, respectively. In addition, this repeatability is observable down to damping values of the order of $10^{-6}$. A summary of these results is presented in Table 2.5. 
Table 2.5: Repeatability measurements of eight beams remounted at least three times with the same clamp setup.

\begin{tabular}{cc|ccc|ccc|c}
\hline & & \multicolumn{3}{|c|}{$\mathbf{1}^{\text {st }}$ Mode Frequency } & \multicolumn{3}{|c|}{ Logarithmic decrement } & \multicolumn{2}{c}{ Error* } \\
Thickness & Length & Min & Mean & Max & Min & Mean & Max & $\delta_{s}$ \\
\hline $\boldsymbol{( \mu \mathbf { m } )}$ & $\mathbf{( m )}$ & & & & & & \\
\hline 73 & 0.01202 & 684.43 & 684.43 & 684.48 & 2.63 & 2.82 & 2.98 & $6.74 \%$ \\
71 & 0.02032 & 221.80 & 221.82 & 221.85 & 1.26 & 1.28 & 1.30 & $1.56 \%$ \\
115 & 0.01577 & 597.66 & 597.80 & 598.02 & 4.20 & 4.51 & 4.78 & $6.87 \%$ \\
115 & 0.01585 & 588.60 & 588.69 & 589.38 & 4.56 & 4.64 & 4.71 & $1.72 \%$ \\
130 & 0.01659 & 569.28 & 569.34 & 569.40 & 4.58 & 4.63 & 4.70 & $1.51 \%$ \\
71 & 0.02579 & 146.18 & 146.36 & 146.73 & 1.17 & 1.17 & 1.18 & $0.85 \%$ \\
42 & 0.02698 & 73.95 & 73.95 & 73.96 & 0.39 & 0.40 & 0.40 & $2.50 \%$ \\
115 & 0.03457 & 128.94 & 128.96 & 128.97 & 2.61 & 2.78 & 2.99 & $7.05 \%$ \\
\hline
\end{tabular}

*The maximum range of all the measurements as a percentage of the mean.

\subsubsection{Measurements of damping}

Figure 2.20 shows a graph of the frequency dependence of the logarithmic decrement of these seventy different single-crystal silicon microcantilevers operating in the first mode. These measurements are plotted as a function of the normalized frequency, $\Omega$, defined in Equation (2.6). Also shown on this graph is the curve corresponding to the prediction of Zener's formula for thermoelastic damping in single-crystal silicon beams. This curve was computed by using Equation (2.6) at $300 \mathrm{~K}$ with the following well-known values for single-crystal silicon: $E=169 \mathrm{GPa}, \alpha=2.6 \times 10^{-6} \mathrm{~K}^{-1}, C=1.6 \times 10^{6} \mathrm{~J} / \mathrm{m}^{3} / \mathrm{K}$, and $k=149 \mathrm{~W} / \mathrm{m} / \mathrm{K}$ [Rei06]. The crystallographic orientation of the silicon beams was taken into account while selecting the value of the Young's modulus [Bra73]. 


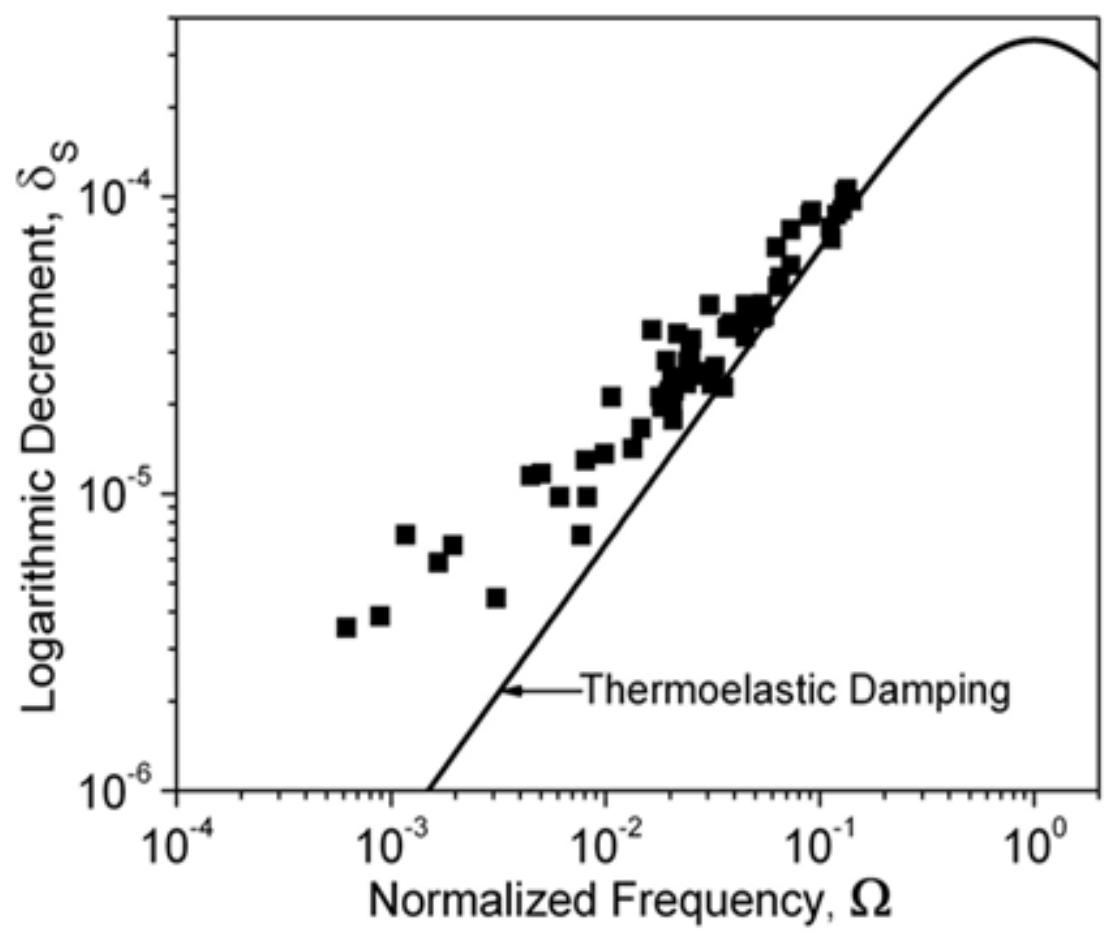

Figure 2.20: Logarithmic decrement measured in 70 different single-crystal silicon microcantilevers operating in the first mode. These measurements are plotted as a function of the normalized frequency, $\Omega$, defined in Equation (2.6). Also shown for comparison is the curve predicted by Zener's formula for thermoelastic damping. Please see Table B1.1 for measurement data

From the measurements shown in Figure 2.20, it is evident that in all cases, the measured damping approaches, but does not decrease below, thermoelastic damping; this behavior is consistent with the expectation that TED establishes the absolute lower bound on damping.

The comparison of the measured damping with predicted TED shows that silicon beams with carefully designed supports and clamps can approach the fundamental limits of dissipation set by thermoelastic damping. The residual damping, (that is, the difference between the measured damping and the predicted thermoelastic damping, $\delta_{s}-\delta_{s, T E D}$ ) varies between $2 \times 10^{-6}$ and $3 \times 10^{-5}$ for the 70 beams shown in this graph. 
Analysis of this losses showed no correlation between this residual damping and beam geometry (length, thickness, and aspect ratios) or frequency, which suggests that support losses are not the main source for the residual damping (see Figure 2.21 and Figure 2.22). In addition, these losses do not correlate with the surface area of the beams as the widths of the beams were nominally similar.

By a process of elimination, we conclude that the residual damping is dominated by clamping losses and is specific to this clamp design. Understanding the source and relations of this loss are a topic of further research.

This clamping loss is constant and specific to each beam. Therefore, this value sets the accuracy with which the beam can be used to measure internal friction in deposited thin films. For all measurements in this thesis, the initial measured damping in the pristine silicon beams $\delta_{s}$ (and not $\delta_{S, T E D}$ ) is used as the reference for all measurements of internal friction in thin films. That is to say 
(a)
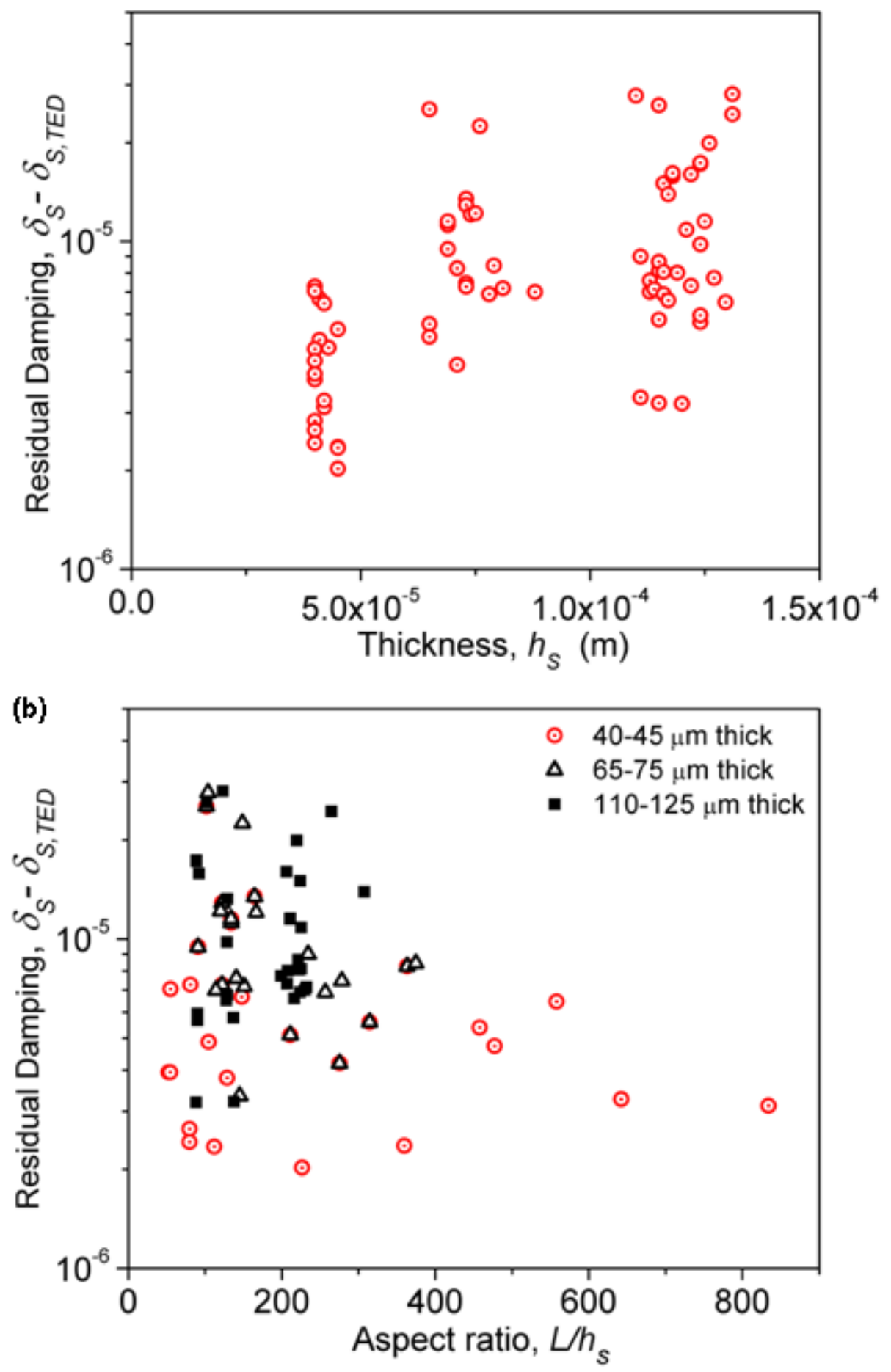

Figure 2.21: Residual damping ( $\left.\delta_{s}-\delta_{s, T E D}\right)$ as a function of a) beam thickness, b) aspect ratio. 
(a)

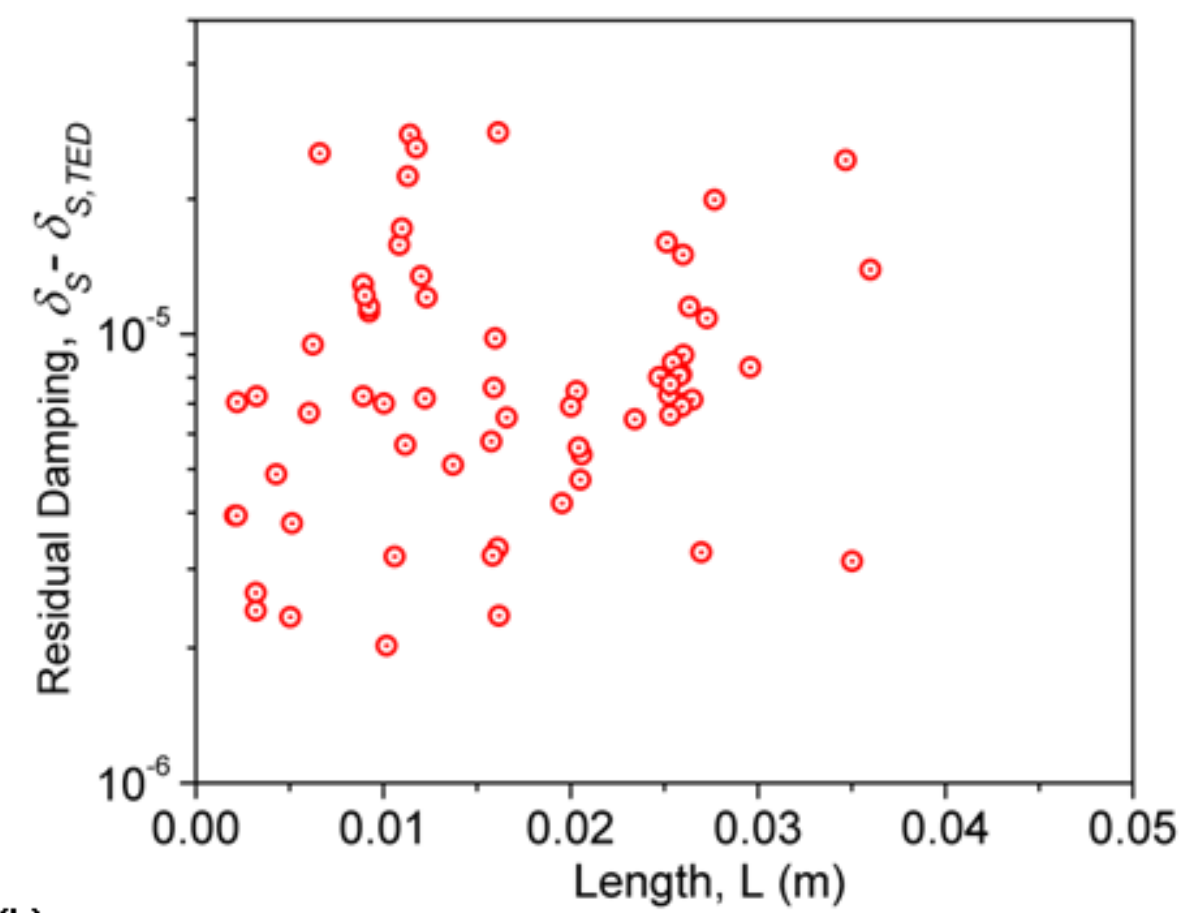

(b)

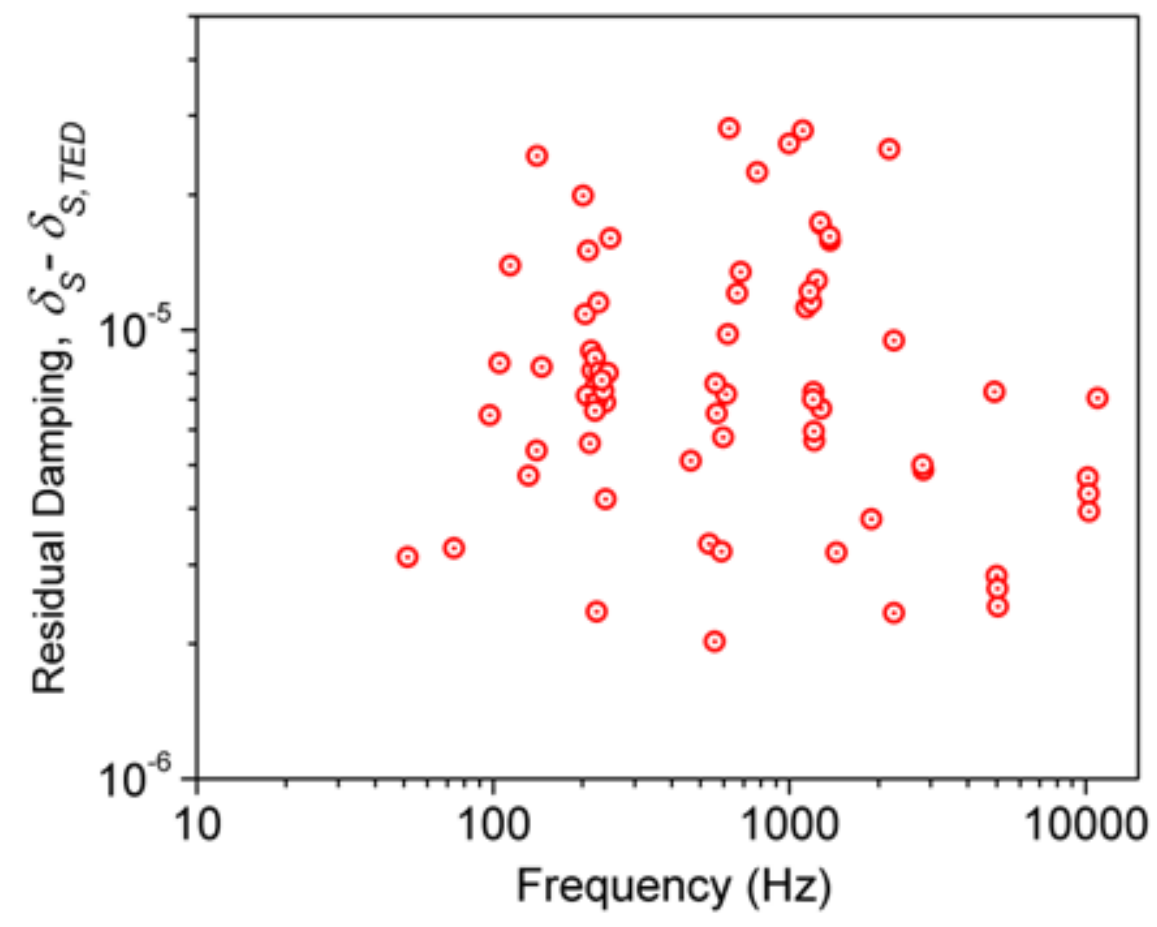

Figure 2.22: Residual damping ( $\left.\delta_{s}-\delta_{s, T E D}\right)$ as a function of a) beam length, b) frequency. 


\subsection{Summary}

This chapter presented a new approach for the accurate measurement of internal friction in thin films. At the heart of this approach is the ability to operate singlecrystal silicon microcantilevers with low damping approaching the fundamental limits of dissipation established by TED and the ability to calculate TED in filmcoated cantilevered resonators using only material properties as the input. The detailed design of a damping measurement platform was described. By systematically minimizing viscous, support, and clamping losses, this system is capable of operating micromachined silicon microcantilevers close to TED (within a difference of $<10^{-5}$ ). By carefully incorporating modifications to make the system repeatable and easily maintainable, the precision of measurement is better than $7 \%$ in measuring damping values of the order of $10^{-5}$. This experimental setup and measurement procedure is used in the subsequent chapters to study dissipation in thin metallic films. 


\section{Chapter 3}

\section{A Study of Internal Friction in Thin films of Aluminum, Silver and Gold at Room Temperature}

\section{Overview}

The first application of the microcantilever platform was to study internal friction in sputter deposited thin films of aluminum, silver and gold. These three metals are widely used in MEMS resonators as functional materials. For instance, aluminum films are widely used in commercial AFM cantilevers to improve reflectivity [Veeco Instruments Inc, Mikromasch USA Inc] while thin films of gold have been used for biological functionalization of cantilevered detectors with self assembled monolayers [Lav04, Lee09].

This chapter looks at a detailed study of the effect of thickness, frequency and adhesion layers of these films on damping. All the films were deposited with relatively low intrinsic stresses $(<160 \mathrm{MPa})$. In addition, the films were characterized by atomic force microscopy (AFM) and scanning electron microscopy (SEM) to analyze the surface structure and topography.

\subsection{Sputter deposition of thin films}

In this study, films of aluminum, silver and gold were deposited on the silicon beams by sputter deposition- a widely used method to deposit thin polycrystalline material films. A typical sputtering machine consists of an anode and cathode separated by a gas filled region. The material to be deposited is termed the target and forms the cathode of the sputter chamber while the substrate to be deposited on is the anode [Mat10]. The basic working principle of sputtering is to bombard

Parts of this chapter appear in a peer reviewed journal publication: G Sosale, S. Prabhakar, L. Frechette, and S. Vengallatore, "A microcantilever platform for measuring internal friction in thin films using thermoelastic damping for calibration," Journal of Microelectromechanical System., vol. 20, pp.764-773 (2011) 
a target material with high energy ionized particles to remove material that is then deposited on the substrate. To create the ions, the sputter chamber is backfilled with a continuous flow of an inert gas such as argon. By initiating a glowdischarge-plasma (a partially ionized gas consisting of positive, negative and neutral particles), the argon atoms are ionized to form positively charged $\left(\mathrm{Ar}^{+}\right)$ ions which are then accelerated at the cathode by a large potential difference between the two electrodes (difference of hundreds to thousands of volts). The subsequent collision with the surface results in a net release of atoms from the cathode into the surrounding gas. These atoms then condense on the surface of the substrate to form the thin film.

Sputter deposition processes are categorized by the power source used to generate the plasma and the way the plasma is manipulated. Details of the various machines and their operating principles are described in detail in several textbooks [Mat10, Ohr02]. The two machines used for deposition of metallic films in this study were MRC 604 (MRC Corporation, USA) and Denton Vacuum Explorer (Denton Vacuum LLC, USA). Both machines were used in the DC sputter mode without the use of any magnetic fields.

For a typical sputter machine, the most commonly used control parameters are i)

the substrate temperature, ii) the argon pressure during deposition, iii) and the power delivered to the plasma. The intricate interplay between these control parameters dictates the nature of the microstructure and, consequently, the properties and functionality of the thin film. One critical property of the film that is closely linked to the microstructure is the internal stress that develops in the film during deposition.

\subsubsection{Internal stresses}

Internal stresses develop in the film during deposition as a result of two different phenomena: thermal mismatch stresses and intrinsic stresses [Nix88]. The former arise from deposition conditions at elevated temperatures where a mismatch in 
thermal expansion coefficients between the substrate and film results in residual stresses as the film cools after deposition. The latter- intrinsic stresses- are dependent on the deposition conditions and are fundamentally related to the accumulation of crystallographic flaws during film growth [Tho89]. For room temperature sputter deposition, higher intrinsic stresses directly correlate with higher defect densities [Ple09, Koc94, Win92] and must therefore be controlled during deposition.

The development of different microstructural features and generation of stress has been shown to depend critically on the ratio of substrate temperature to the melting temperature of the target material ( $\mathrm{T} / \mathrm{T}_{\text {melting }}$ ) [Tho77]. For low temperature depositions $\left(\mathrm{T} / \mathrm{T}_{\text {melting }}<0.3\right)$, several other deposition factors come into play such as [Win92]: i) the deposition pressure, ii) deposition temperature of the substrate, iii) substrate bias, iv) target gas mass ratios v) substrate orientation and vi) cathode shape. Of these, the most widely studied has been the effect of gas pressure. Figure 3.1 shows a schematic representation of a typical process-property correlation curve for stress as a function of the argon pressure during deposition [Win92].

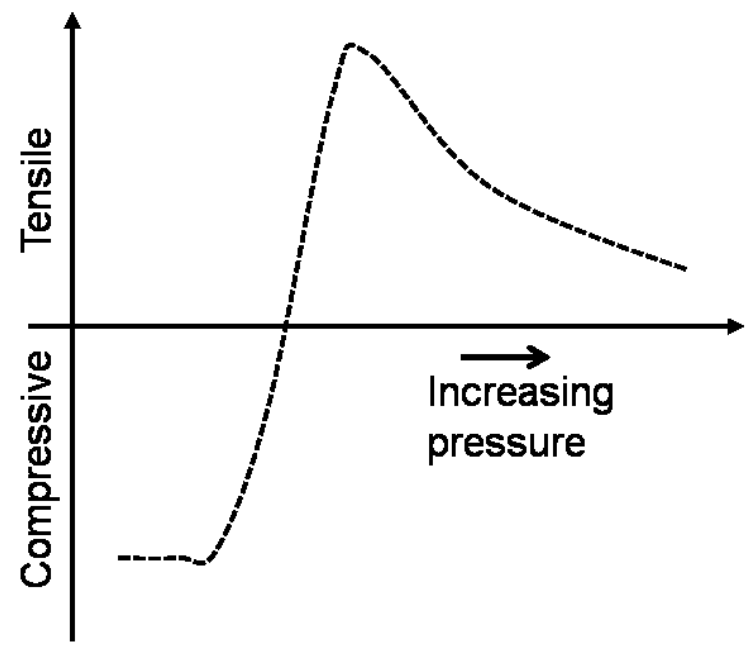

Figure 3.1: Schematic of the effect of deposition pressure on the stress in sputter deposited thin films [Win92]. 
For sputtered aluminum films, an exhaustive literature search revealed only a few studies that examine the intrinsic stress properties of sputter deposited aluminum films that were less than $1 \mu \mathrm{m}$ in thickness [Ple09, Kim98, Hof77] and even fewer were found for gold [Keb90] and silver [Mar94]. These studies provided the starting parameters for deposition conditions; however, the variations in machines made the direct translation of process parameters difficult. Therefore before carrying out any damping studies, the films used in this experiment were first characterized for stress and microstructure. The aim was to obtain low and comparatively similar stresses in the thin films of Al, Ag and Au.

\subsubsection{Measuring film stress}

A common approach to measuring film stress is to examine the change in curvature of a standard single-crystal silicon wafer with the addition of a thin film [Ohr02]. This approach is illustrated by the schematic cross-sectional diagrams shown in Figure 3.2. After deposition, the film has an unconstrained length $\mathrm{L}_{\mathrm{f}}$ that is in general different from the length of the silicon substrate However, when deposited on the relatively thick substrate, and assuming perfect adhesion between
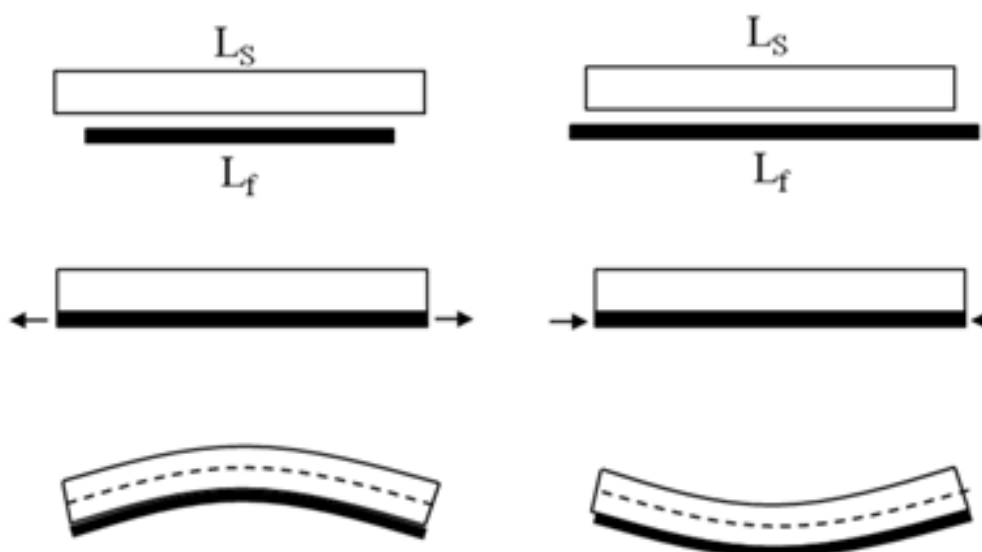

Tensile stresses

in Film
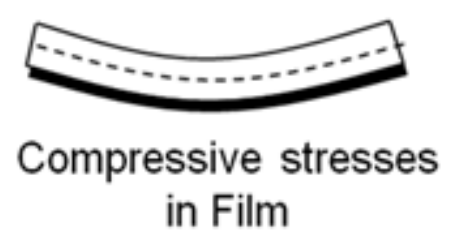

Figure 3.2: Illustration of the effect of internal stress of a thin film on a thick substrate. A thin film with internal stresses is stretched or compressed to match the strain in the substrate. The resulting force of the film is balanced by curvature in the substrate [Mad02]. 
the film and substrate, the film is either stretched or compressed to a length $\mathrm{L}_{\mathrm{S}}$. The net result is the generation of stresses in the substrate that balance those in the film, leading to a curvature of the film-substrate composite, as shown in the figure.

In the limit of a thin film on a thick substrate, the change in curvature of the composite is related to the stress in the film by an expression first derived by Stoney [Sto09]:

$$
\sigma_{f}=\frac{E_{S} h_{S}^{2}}{6 h_{f}}\left[\frac{1}{R_{C}}-\frac{1}{R_{S}}\right],
$$

where $E_{S}$ is the Young's modulus of the substrate, $R_{S}$ is the initial curvature of the wafer, $R_{C}$ is the final curvature of the film-substrate composite and $h_{S}$ and $h_{f}$ are the substrate and film thickness respectively. For two dimensional stresses such as those on a wafer, an additional correction is to use the biaxial modulus to yield [Ohr02]

$$
\sigma_{f}=\frac{E_{S} h_{S}^{2}}{6(1-v) h_{f}}\left[\frac{1}{R_{C}}-\frac{1}{R_{S}}\right],
$$

where $v$ is the Poisson's ratio of the substrate. From Equation (3.2) we see that the mechanical properties of the film are not required to estimate stress. However, accurate measurements of the film thickness, substrate thickness and change in curvature are imperative. For very thin films $\left(h_{f}<20 \mathrm{~nm}\right)$ on a standard silicon $\left(h_{S} \sim 500 \mu \mathrm{m}\right)$ wafer, the accuracy of stress calculations is limited by the accuracy with which the film thickness can be characterized. Thus, a variation of $1 \mathrm{~nm}$ in measurement at these film thicknesses would imply a $40 \mathrm{MPa}$ variance in the calculated stress, regardless of the resolution of the measurement of curvature.

In this study, curvature measurements were made with a Flexus 5200 (KLATencor Inc, CA, USA) laser based measurement system. Film thickness was measured using an Ambios XP 200 Profiler (KLA-Tencor Inc, CA, USA) which has a vertical height resolution of $1 \mathrm{~nm}$. For films below $50 \mathrm{~nm}$ in thickness, 
additional checks were carried out with an atomic force microscope to verify the values obtained from the profiler.

\subsection{Characterization of stress and surface topography in sputtered thin films of aluminum, silver and gold}

\subsubsection{Stress measurements}

Before any measurements of damping were carried out, the films were characterized for intrinsic stresses, surface topography and a qualitative examination of the microstructure. All depositions were carried out on 4" wafers that were nominally $514-525 \mu \mathrm{m}$ thick. The wafers were first cleaned in a Piranha solution $\left(1: 8, \mathrm{H}_{2} \mathrm{O}_{2}: \mathrm{H}_{2} \mathrm{SO}_{4}\right)$ to remove any organic residues and then thoroughly rinsed and dried. The initial curvature of all wafers was then measured and recorded. To start with, deposition parameters previously used in literature [Kim98, Keb90, Mar94] were used for sputtering. The films were deposited using DC sputtering without actively heating or cooling the substrates, and using metal targets with purity greater than $99.95 \%$. In order to ensure good adhesion, thin adhesion layers $(\sim 10-15 \mathrm{~nm})$ of chromium and titanium were first sputter deposited on the wafers and then subsequently coated with Al, Ag or Au without breaking vacuum. Initial trials were carried out to obtain films of approximately $60,110,220$ and $450 \mathrm{~nm}$ in thickness. The repeatability of the deposition process was then analyzed by measuring the stress values for three consecutive runs for the same deposition time. Two wafers were used for each run and compared. The maximum run-to-run variation was approximately $15 \%$ of the average value. The maximum run-to-run variation in thickness was found to be approximately $3 \%$.

\subsubsection{Result of stress measurements for Al, Ag and Au films}

Let us first examine the results for the aluminum film without the adhesion layer. Figure 3.3 shows the intrinsic stresses in the thin films of aluminum (without chromium) sputter deposited at the same deposition power but at two different pressures (10 mTorr and $25 \mathrm{mT}$ Torr). From the figure we see that an increase in 
pressure decreased the intrinsic stress in the film. For films thicker than $100 \mathrm{~nm}$, the films deposited at $10 \mathrm{mTorr}$ pressure had a progressively more cloudy appearance. However, at 25 mTorr the shiny appearance and reflectivity was maintained across the entire range of thickness. Also shown in Figure 3.3 is the stress in $15 \mathrm{~nm}$ of chromium and its effect on the overall stress in the aluminum/chromium $(\mathrm{Al} / \mathrm{Cr})$ thin film. With the addition of chromium, there is almost an order of magnitude increase in the stress for the $60 \mathrm{~nm} \mathrm{A1/Cr}$ film. The Cr films are highly stressed: a $15 \mathrm{~nm}$ film has approximately $350 \mathrm{MPa}( \pm 50 \mathrm{MPa})$ of stress. However, as the $\mathrm{Al} / \mathrm{Cr}$ films grow in thickness, the stress decreases suggesting the $\mathrm{Cr}$ layer is the largest contributor to the increased stress in the films.

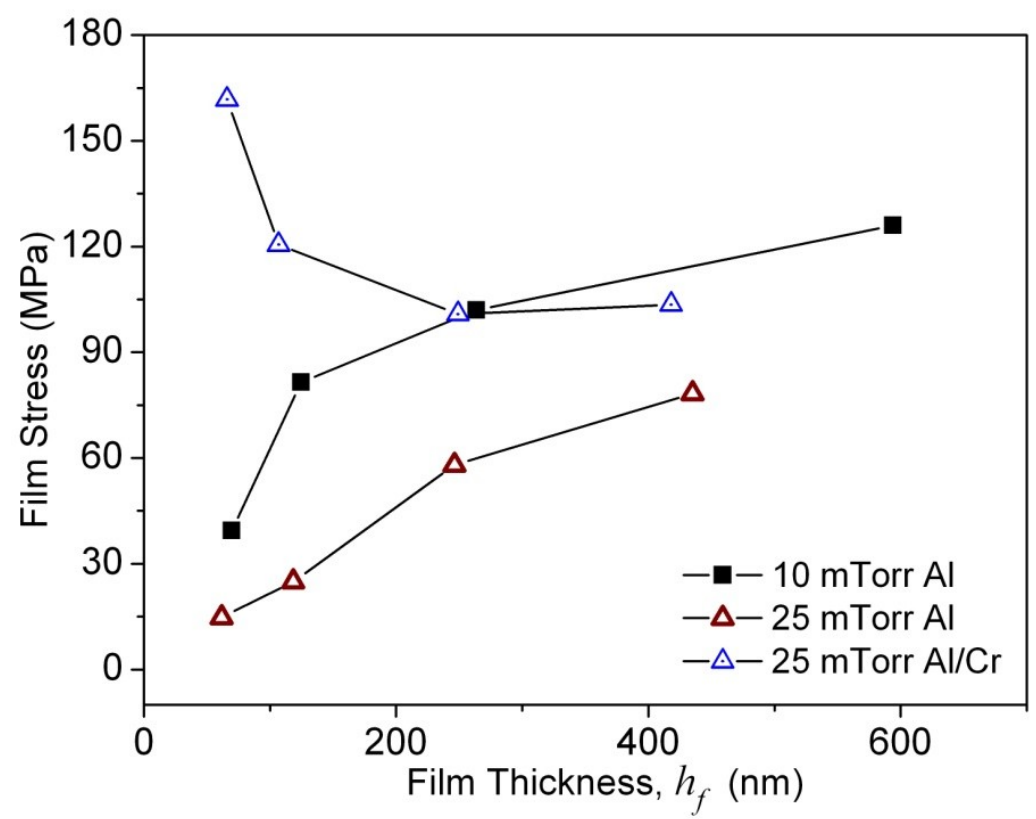

Figure 3.3: Effect of argon pressure on the film stress in $\mathrm{Al}$ and $\mathrm{Al} / \mathrm{Cr}$ films. $\mathrm{Al}$ was deposited at a power density of $4.5 \mathrm{~W} / \mathrm{cm}^{2}$. The $\mathrm{Cr}$ layer is $\sim 15 \mathrm{~nm}$ thick, has a stress of $350 \pm 50 \mathrm{MPa}$ and was deposited at a power density of $2.4 \mathrm{~W} / \mathrm{cm}^{2}$. The relative error in measurements is $\pm 15 \%$.

Figure 3.4 shows the stress as a function of thickness for gold and silver with a 15 $\mathrm{nm}$ adhesion layer of titanium. Both $\mathrm{Ag} / \mathrm{Ti}$ and $\mathrm{Au} / \mathrm{Ti}$ films had stresses that ranged between $50 \mathrm{MPa}$ in compression to $75 \mathrm{MPa}$ in tension and there was no 
measureable effect of the adhesion layer. A summary of the final deposition parameters used in this study is provided in Table 3.1.

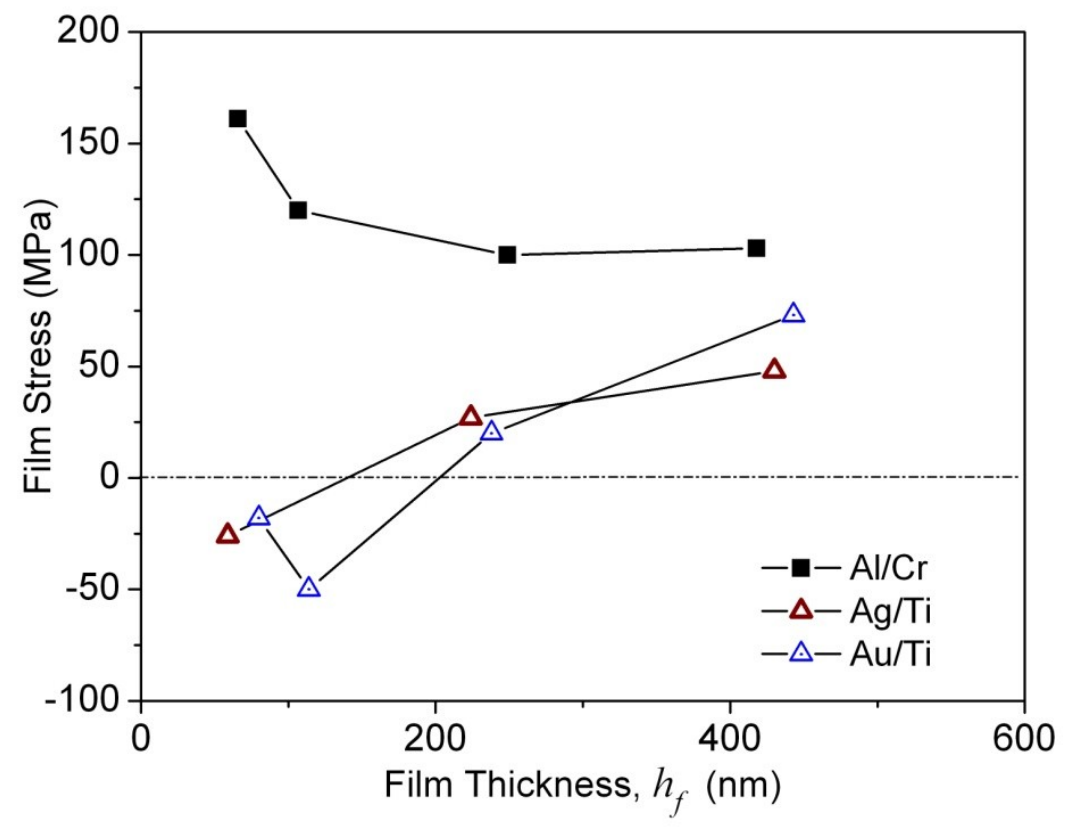

Figure 3.4: Plot of intrinsic stress in aluminum, silver and gold films under the conditions in Table 3.1. All films were deposited with a $\sim 15 \mathrm{~nm}$ adhesion layer of chromium (for aluminum) or titanium (for silver and gold). The relative error in the measurements is $\pm 15 \%$.

Table 3.1: Deposition parameters and measured residual stress for the sputtered thin films.

\begin{tabular}{ccccc}
\hline Material (thickness) & $\begin{array}{c}\text { Power } \\
\text { Density } \\
\left(\mathbf{W} / \mathbf{c m}^{2}\right)\end{array}$ & $\begin{array}{c}\text { Deposition } \\
\text { Pressure } \\
(\mathbf{m T o r r})\end{array}$ & $\begin{array}{c}\text { Deposition } \\
\text { Rate } \\
(\mathbf{n m} / \mathbf{s})\end{array}$ & $\begin{array}{c}\text { Maximum } \\
\text { stress } \\
( \pm 15 \%)\end{array}$ \\
\hline $\mathrm{Cr}(15 \mathrm{~nm})$ & 2.4 & 25 & 3.0 & $360 \mathrm{MPa}^{*}$ \\
$\mathrm{Ti}(15 \mathrm{~nm})$ & 1.1 & 10 & 0.1 & $<40 \mathrm{MPa}$ \\
$\mathrm{Cr}(15 \mathrm{~nm})+\mathrm{Al}(60 \mathrm{~nm})$ & 4.5 & 25 & 3.0 & $160 \mathrm{MPa}$ \\
$\mathrm{Ti}(15 \mathrm{~nm})+\mathrm{Ag}(430 \mathrm{~nm})$ & 0.58 & 10 & 0.5 & $50 \mathrm{MPa}$ \\
$\mathrm{Ti}(15 \mathrm{~nm})+\mathrm{Au}(440 \mathrm{~nm})$ & 0.58 & 10 & 0.5 & $75 \mathrm{MPa}$ \\
\hline
\end{tabular}

$*$ The resolution of the measurement is $\pm 50 \mathrm{MPa}$ at this thickness. 


\subsubsection{Surface topography characterization with AFM}

A representative wafer for each thickness of film was then cleaved and a piece of the wafer used to obtain the surface topography of the film. The films were imaged by atomic force microscopy to examine the surface topography. The AFM measurements were carried out with a Jeol 5200 SPM (Jeol Inc, USA) and using cantilevers (NSC15/AlBS tips, Mikromasch USA) with a tip radius less than 10 $\mathrm{nm}$ and an aluminum backside coating to improve the reflectivity. The images were acquired in tapping mode for a scan size $1.5 \times 1.5 \mu \mathrm{m}$ and at multiple locations on the surface of films.

For all surfaces, both topography and phase lag images were obtained. In tapping mode, topography images are obtained from the change in amplitude of the oscillation of the cantilever and provide quantitative information on the spatial variation in height on the surface [Mey04]. On the other hand, phase lag images are produced by the change in phase between the driving signal given to the cantilever and the response of the cantilever. The phase lag provides information

on the dissipation between the surface and the tip. Phase images are very sensitive to material in-homogeneities and have been shown to resolve the grain structure on the surface of thin films [Pan02].

The AFM is a powerful tool to image the surface. However, any measurement may contain errors such as ringing noise, tip convolutions and distortions due to scanning irregularities [Mey04]. Therefore, to qualitatively confirm the AFM measurements, scanning electron micrographs (SEM) of the surfaces of the aluminum films were obtained at a similar magnification.

\section{Surface topography of aluminum films}

Figure 3.5 and 3.6 show representative topography and phase lag images for the $60 \mathrm{~nm}$ and $430 \mathrm{~nm}$ thick films of aluminum. From measurements of surface 
topography, the root-mean-squared surface roughness increases from $8 \mathrm{~nm}$ to 23 $\mathrm{nm}$ over the thickness range. In comparison to the topography images, the phase lag images clearly delineate the surface grains. For the Al films, we see that the grain size is of the order of the thickness of the film and increases with film thickness. Also shown are SEM micrographs of the same surfaces. Qualitatively, the SEM images matched well with the AFM images confirming that tip artifacts are minimized
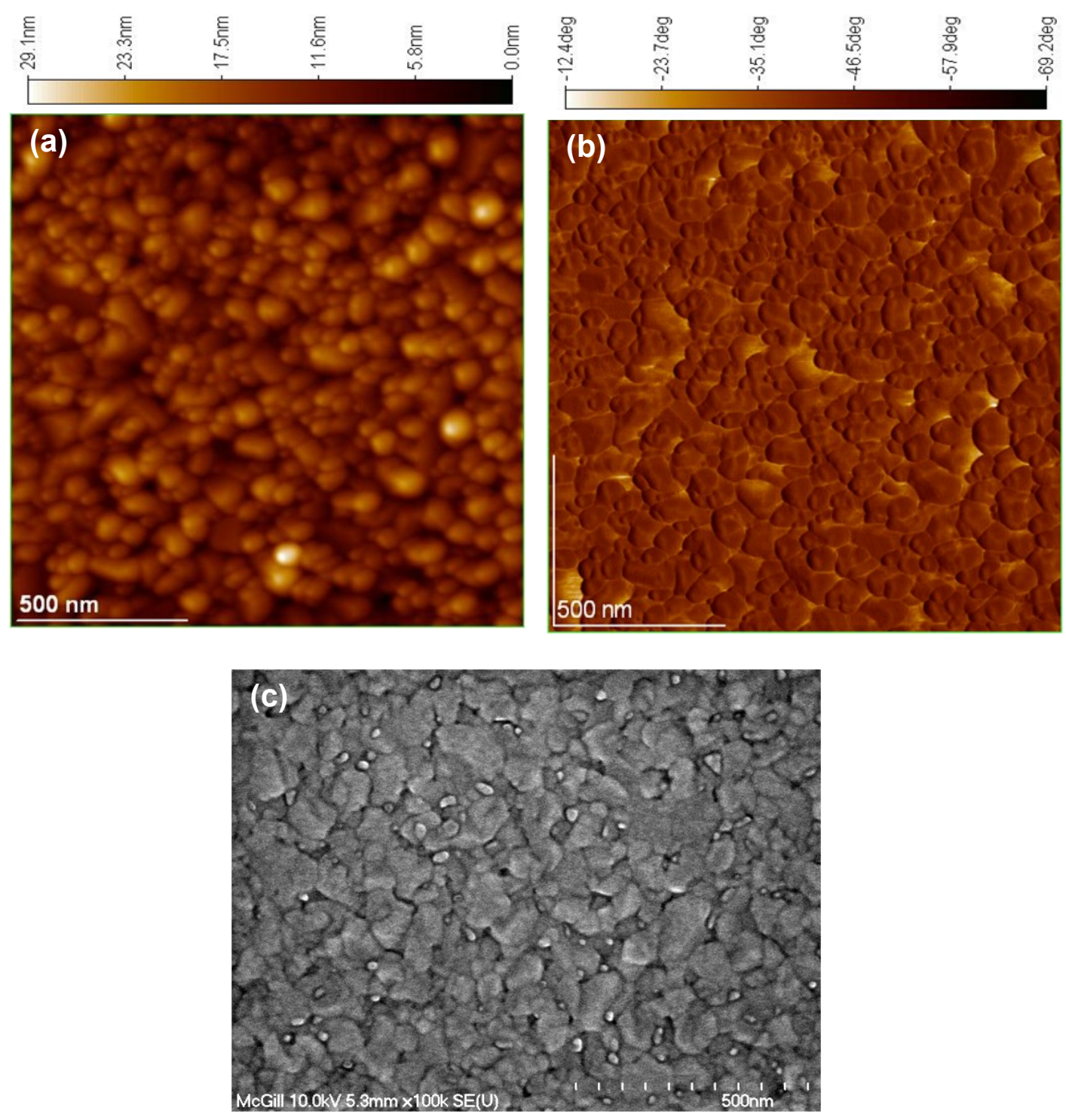

Figure 3.5: $1.5 \times 1.5 \mu \mathrm{m}^{2}$ atomic force microscopy (AFM) images of $60 \mathrm{~nm}$ thick aluminum film: a) topography, b) phase image, c) scanning electron micrograph of the film surface at a similar scale. 

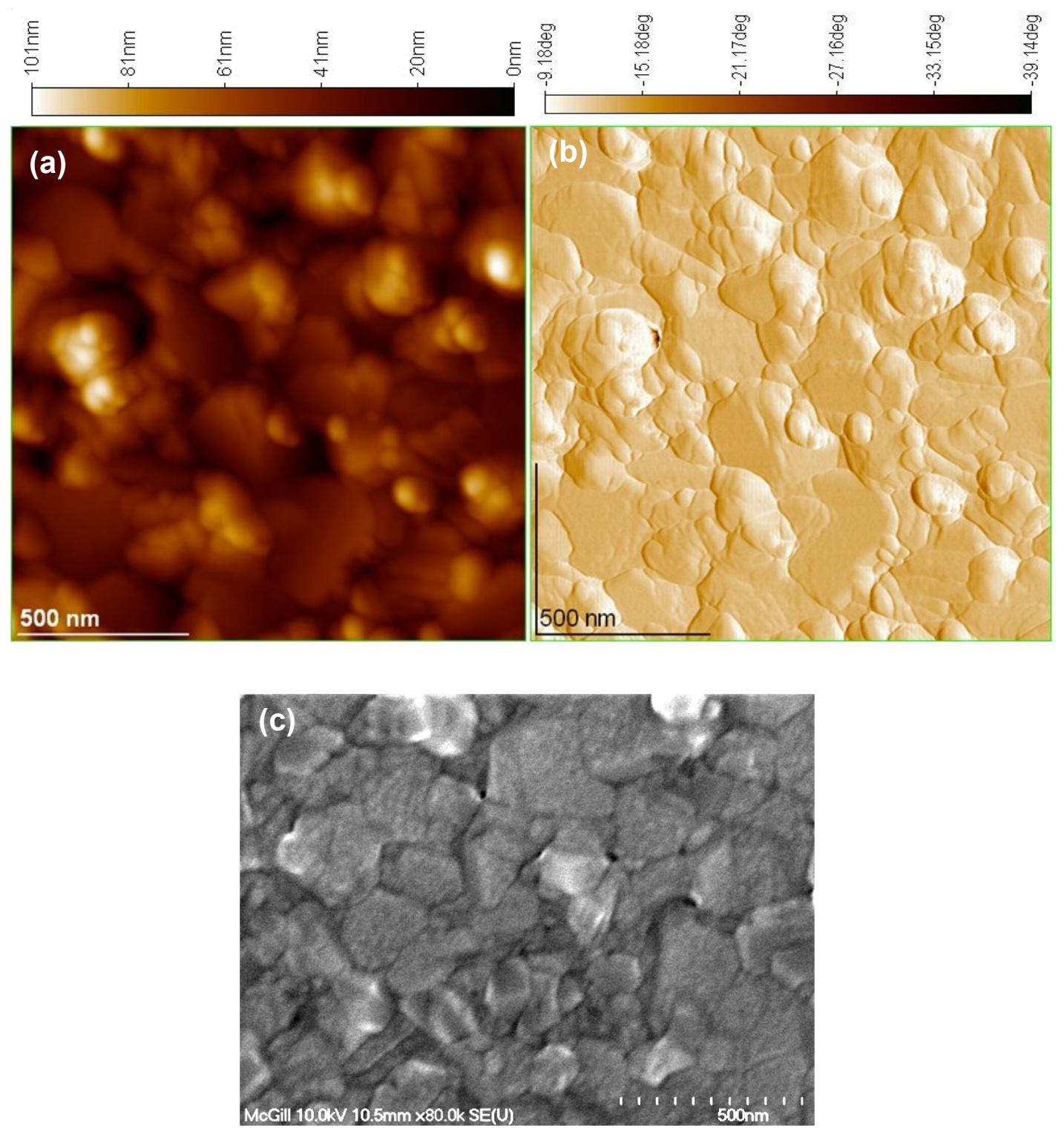

Figure 3.6: $1.5 \times 1.5 \mu \mathrm{m}^{2}$ Atomic force microscopy (AFM) images of $430 \mathrm{~nm}$ thick aluminum film: a) topography, b) phase image. Also shown is c) a scanning electron micrograph of the film surface at a similar scale.

\section{Surface topography and phase images of silver and gold films}

The Figures 3.7 and 3.8 show representative AFM images for silver and gold films at thicknesses of $\sim 60$ and $\sim 400 \mathrm{~nm}$ respectively. The roughness of these films was similar to that of the aluminum films $(\sim 8 \mathrm{~nm}$ for the $\sim 60 \mathrm{~nm}$ films to $\sim$ 
$25 \mathrm{~nm}$ for the $\sim 400 \mathrm{~nm}$ films). The images show that the grain size in these films increases with film thickness.
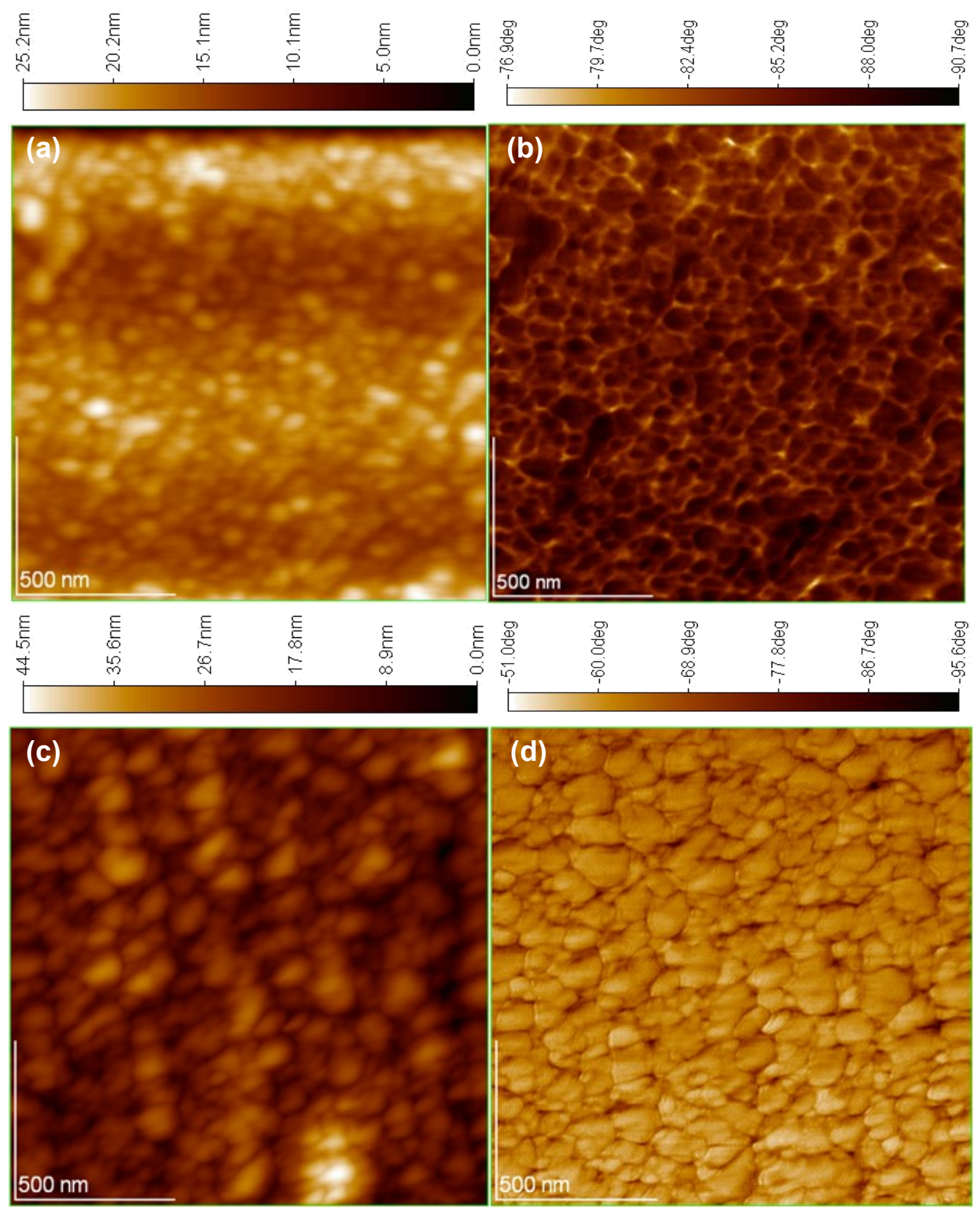

Figure 3.7: Representative $1.5 \times 1.5 \mu \mathrm{m}^{2}$ AFM images of silver films: a) topography b) phase lag image of $60 \mathrm{~nm}$ thick silver film, c) topography, d) phase image of $430 \mathrm{~nm}$ thick film. 


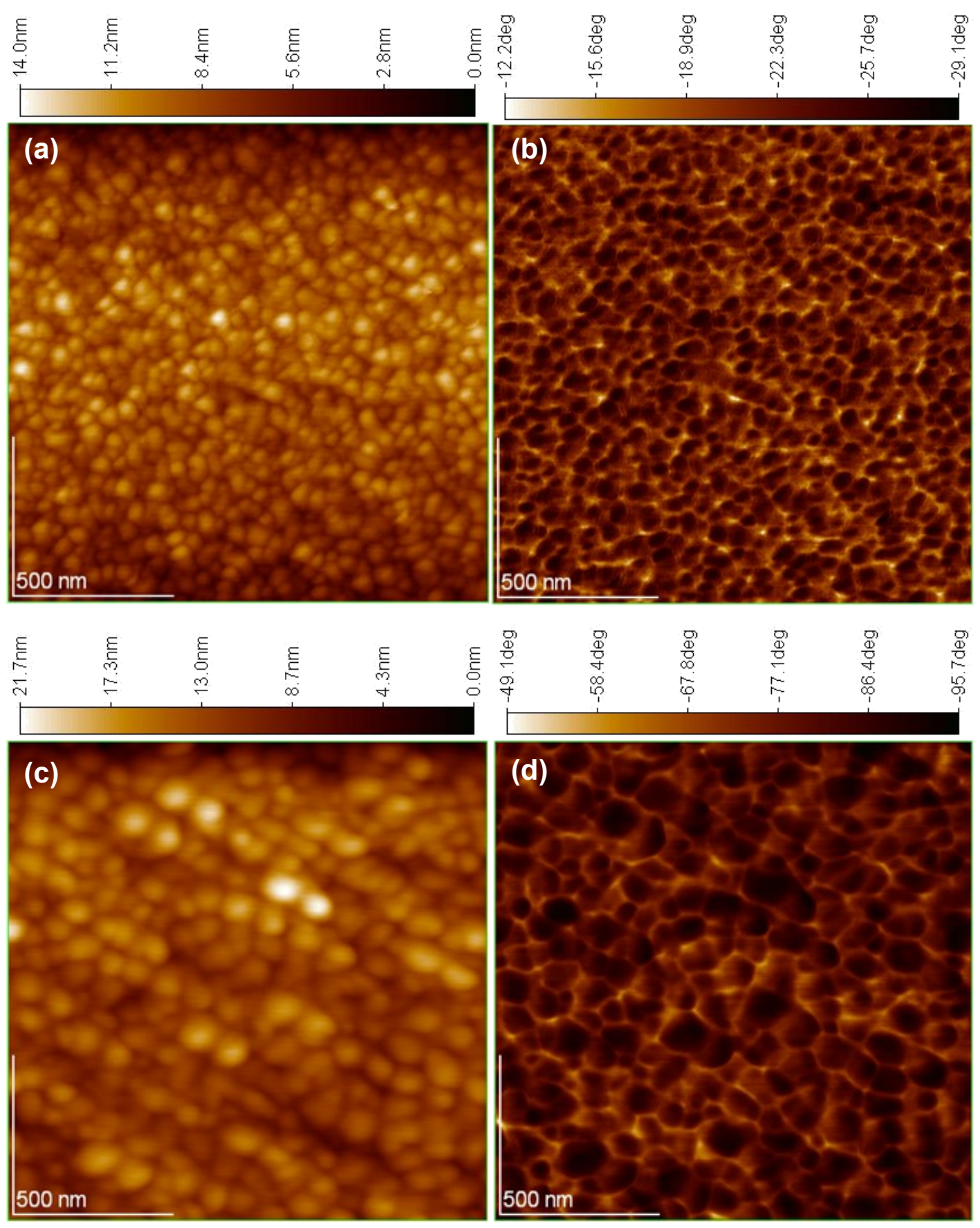

Figure 3.8: Representative $1.5 \times 1.5 \mu \mathrm{m}^{2}$ AFM images of gold films: a) topography b) phase lag image of $60 \mathrm{~nm}$ thick silver film, c) topography, d) phase image of $440 \mathrm{~nm}$ thick film.

Taken together, Figures 3.5 to 3.8 provide a description of the microstructure of the film. In all cases, the average grain size increases with film thickness. At a thickness of $\sim 60 \mathrm{~nm}$, the grain sizes of aluminum, silver and gold are similar. 
However, at $\sim 400 \mathrm{~nm}$ the silver and gold films appear to have a wider distribution of grain size in comparison to aluminum films of the same thickness. The next section looks at measurements of damping in these films.

\subsection{Measurement of damping in aluminum, silver and gold films}

For damping studies, films of aluminum, gold, silver, chromium, and titanium were deposited on subset of 46 single-crystal silicon beams whose properties were described earlier in section 2.5. These beams ranged from 42 to $131 \mu \mathrm{m}$ in thickness, 0.9 to $1.1 \mathrm{~mm}$ in width, and 0.9 to $3.6 \mathrm{~cm}$ in length. The length-tothickness aspect ratio of these beams ranged from 88 to 557, and their fundamental natural frequency for bending vibration ranged from 97.5 to 1445 $\mathrm{Hz}$. The face-centered cubic (fcc) metals ( $\mathrm{Al}, \mathrm{Au}$, and $\mathrm{Ag}$ ) were deposited for average film thicknesses of approximately $60,110,240$ and $430 \mathrm{~nm}$ to examine the effects of thickness on internal friction. Films were deposited on one surface of the single-crystal silicon microcantilevers. To prevent any change in clamping losses due to metallization, care was taken to ensure that the clamping and supporting areas remained free of any coatings. In order to do this, a custom machined molybdenum clamp was used to hold the beams as shown in Figure 3.9. After deposition, the damping in the composite beam was measured and the change in TED due to addition of the film calculated.

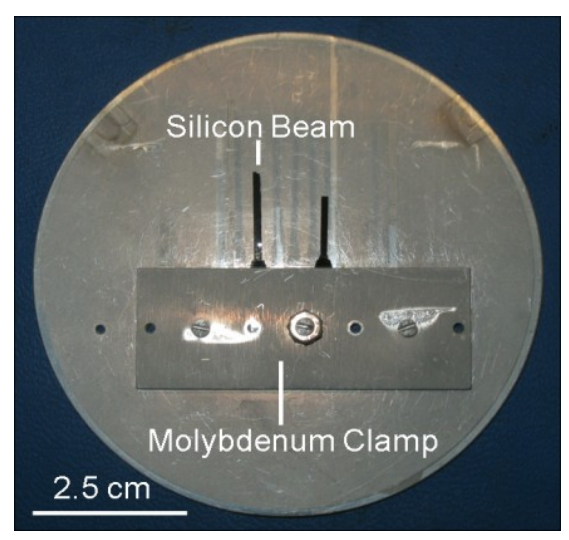

Figure 3.9: Photograph of custom machined molybdenum clamp and wafer for sputter deposition of films onto the beams. To prevent shadowing effects, the small part of the base is exposed during deposition. 


\subsubsection{Results}

First, six beams were used to quantify the effects of the $15 \mathrm{~nm}$ thick adhesion layers of $\mathrm{Cr}$ and $\mathrm{Ti}$ on damping. Figure 3.10 shows the measured values of damping in the bare silicon microcantilevers and in the $\mathrm{Cr} / \mathrm{Si}$ and $\mathrm{Ti} / \mathrm{Si}$ composites. The change in damping after metallization with the adhesion layers was below the resolution of measurement.

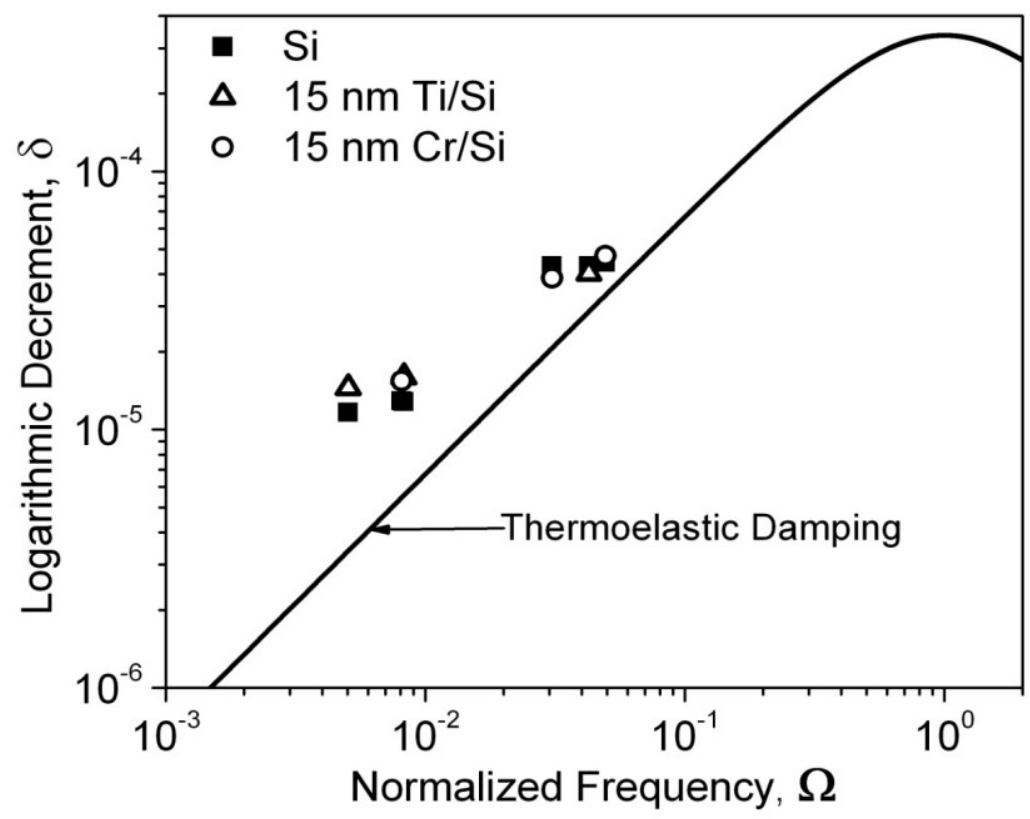

Figure 3.10: Effect of thin adhesion layers ( $15 \mathrm{~nm} \mathrm{Ti,} 15 \mathrm{~nm} \mathrm{Cr}$ ) on damping of single-crystal silicon microcantilevers.

Next, we consider the effects of thickness and frequency on damping in thin films of aluminum, silver and gold. In all cases, $15 \mathrm{~nm}$ thick films of $\mathrm{Cr}$ and Ti were used to ensure adhesion of the fcc metals to silicon. Figure 3.11 shows the measured increase in damping, $\delta_{c}-\delta_{s}$, as a function of the thickness of the metals. All measurements were made at room temperature by exciting the composite microcantilevers at a frequency close to their fundamental natural frequencies, and then analyzing the free decay of these resonators. The fundamental natural frequency of the silicon beams that were used for these 
measurements ranged from $203 \mathrm{~Hz}$ to $248 \mathrm{~Hz}$. The change in frequency after coating with thin films (50 nm to $500 \mathrm{~nm}$ ) of $\mathrm{Al}, \mathrm{Ag}$, and $\mathrm{Au}$ was measured to be negligibly small (typically, less than $0.5 \%$ ). This is consistent with the predictions of a model for the vibrations of a slender bilayer consisting of a thin film on a thick substrate [Ber75].

The results shown in Figure 3.11 indicate that damping in the composite microcantilever increases monotonically with film thickness. For any given thickness, aluminum dissipates more energy than gold. Indeed, the change in damping due to the $60 \mathrm{~nm}$ thick gold film is at the limit of resolution. The same dependence on thickness was observed at all frequencies $(97.5 \mathrm{~Hz}$ to $1445 \mathrm{~Hz}$ ) measured in this study.

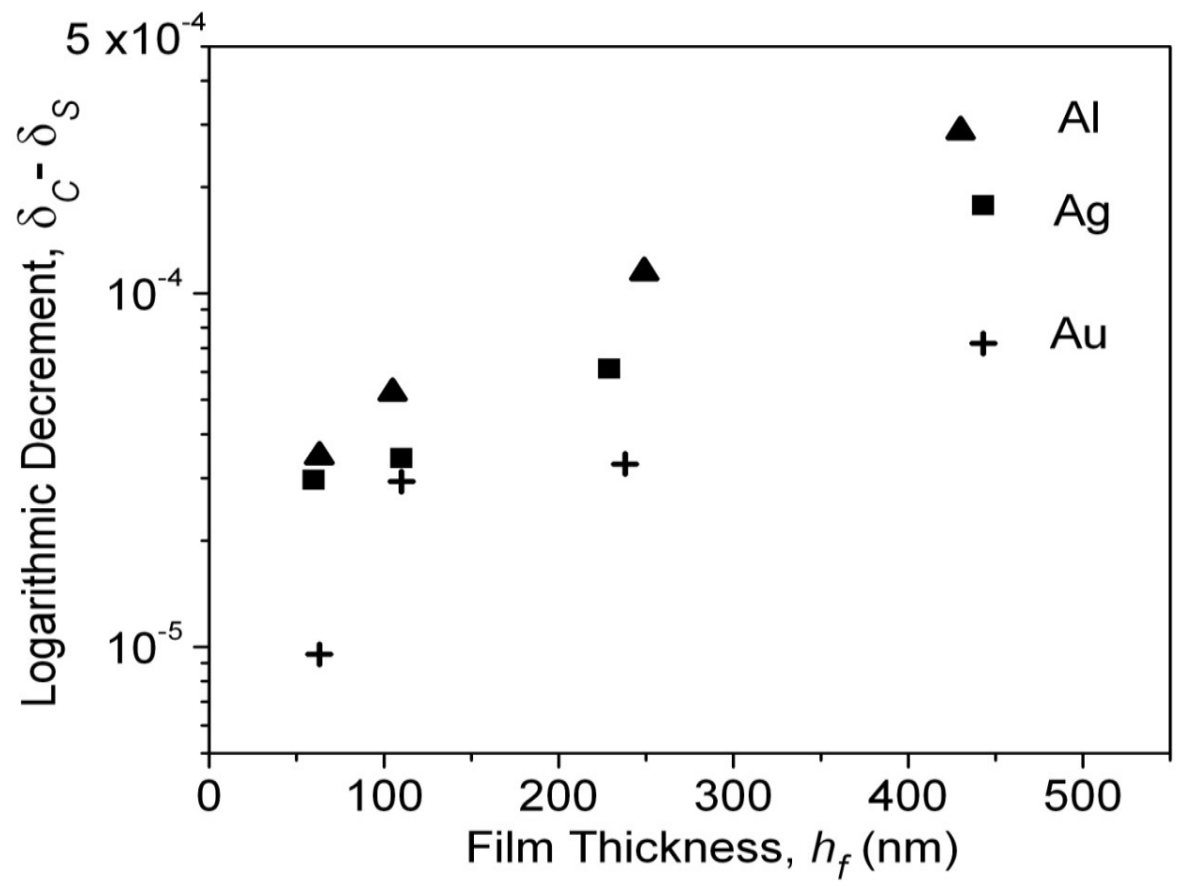

Figure 3.11: Increase in damping due to metallization with thin films of aluminum $(\boldsymbol{\Delta})$, silver $(\boldsymbol{\square})$ and gold $(+)$. The natural frequency of the silicon beams used for these measurements varied over a narrow range between $203 \mathrm{~Hz}$ and 248 Hz. In all cases, $15 \mathrm{~nm}$ thick adhesion layers of $\mathrm{Cr}$ and $\mathrm{Ti}$ were used to ensure adhesion of $\mathrm{Al}, \mathrm{Au}$, and $\mathrm{Ag}$ to silicon. 
The change in damping after metallization was used to estimate the internal friction in the thin films. A check was carried out to verify that the change in TED due to metallization was negligible [Pra08], and then the internal friction in $\mathrm{Al}$, $\mathrm{Ag}$, and $\mathrm{Au}$ was calculated using Equation (2.9) and the following values: $E_{A l}=70$ $\mathrm{GPa}, E_{A u}=82 \mathrm{GPa}, E_{A g}=76 \mathrm{GPa}, E_{S i}=169 \mathrm{GPa}, \rho_{A l}=2.7 \times 10^{3} \mathrm{~kg} / \mathrm{m}^{3}$, $\rho_{A u}=1.93 \times 10^{4} \mathrm{~kg} / \mathrm{m}^{3}, \quad \rho_{A g}=1.05 \times 10^{4} \mathrm{~kg} / \mathrm{m}^{3}$ and $\rho_{A l}=2.3 \times 10^{3} \mathrm{~kg} / \mathrm{m}^{3}$. Representative results for the effects of thickness and frequency on internal friction are shown in Fig. 3.12 and Fig. 3.13, respectively. The highest values of internal friction were obtained for the thinnest films $(60 \mathrm{~nm})$ and lowest frequencies $(\sim 100 \mathrm{~Hz})$ measured in this study with films of aluminum and silver. For example, internal friction in the $60 \mathrm{~nm}$ thick film of $\mathrm{Ag}$ was about twice that measured in films with thickness of $230 \mathrm{~nm}$ and $450 \mathrm{~nm}$. In contrast, there was no significant change in the internal friction of gold films when the thickness was reduced from $450 \mathrm{~nm}$ to $60 \mathrm{~nm}$.

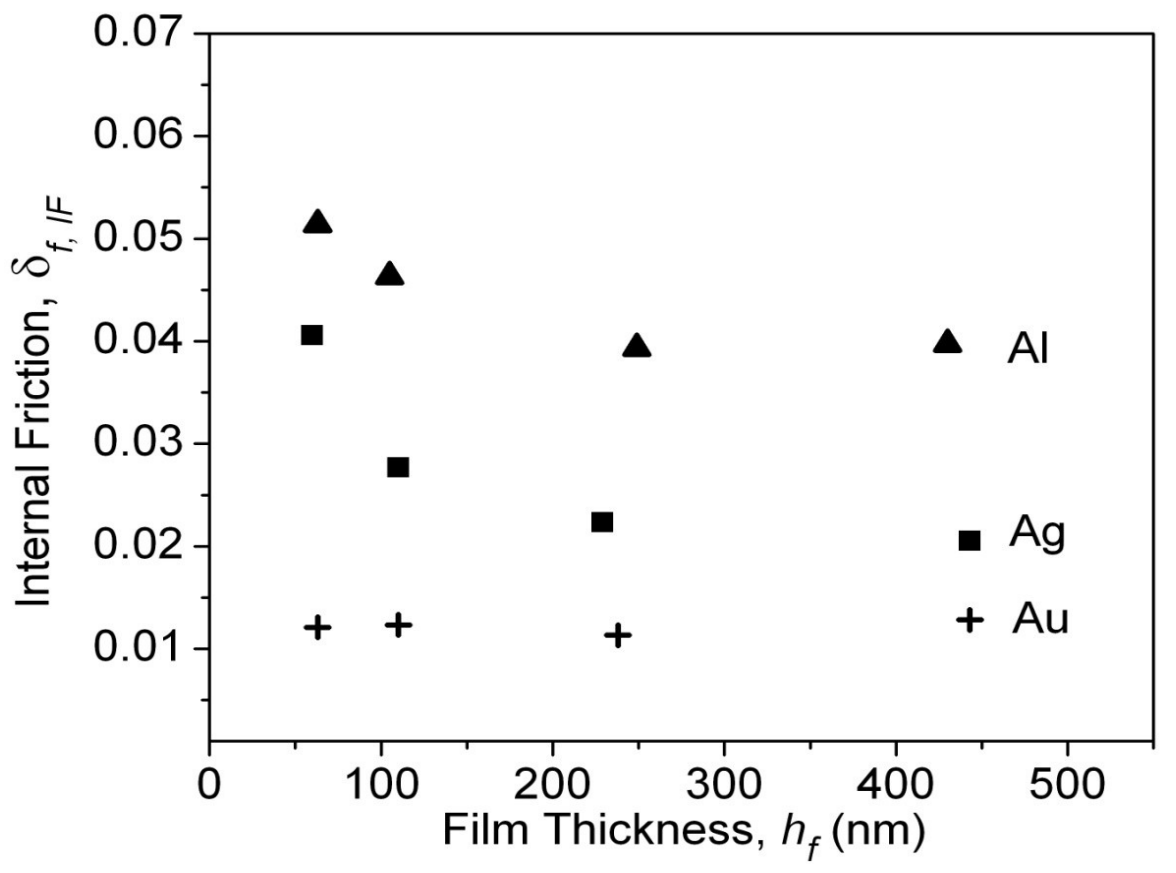

Figure 3.12: Effect of thickness on internal friction in thin films of aluminum $(\boldsymbol{\Delta})$, silver $(\boldsymbol{\square})$ and gold $(+)$. All measurements were taken over a relatively narrow range of frequencies between $203 \mathrm{~Hz}$ and $248 \mathrm{~Hz}$. The relative error of the internal friction values is $\pm 15 \%$. 


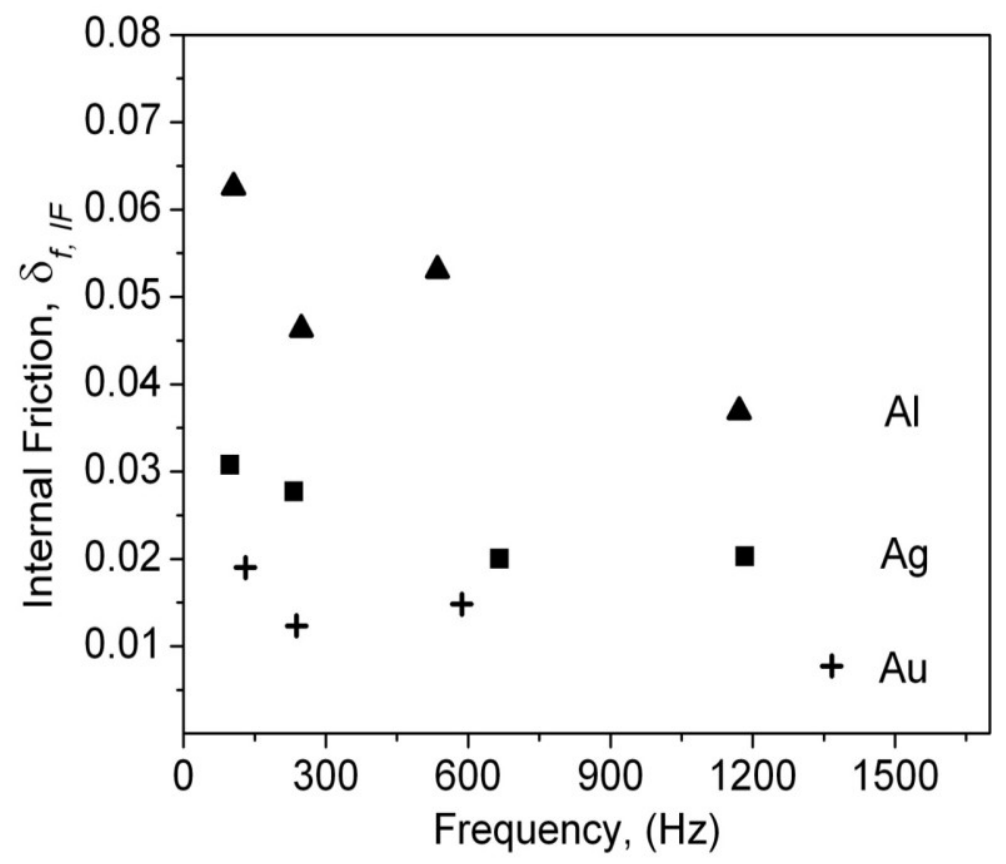

Figure 3.13: Frequency dependence of internal friction in $110 \mathrm{~nm}$ thick films of aluminum $(\boldsymbol{\Delta})$, silver $(\boldsymbol{\square})$ and gold $(+)$. The relative error of the internal friction values is $\pm 15 \%$.

\subsubsection{Discussion}

The damping in the metalized beams increased monotonically as the thickness of $\mathrm{Al}, \mathrm{Ag}$, and $\mathrm{Au}$ increased from $50 \mathrm{~nm}$ to $500 \mathrm{~nm}$, as shown in Figure 3.11. We conclude from this that the dissipation is dominated by processes occurring within the film, and not at the metal/silicon interface or at the free surface of the film. Indeed, if surface or interfacial processes were dominant, the damping in the layered composite will be expected to be independent of film thickness. This was not observed at any frequency in these measurements.

The lack of dissipation due to interfacial sliding was expected because of the use of $\mathrm{Ti}$ and $\mathrm{Cr}$ to ensure adhesion between the metals and silicon, and also because of the lack of any significant shear stress at the metal/silicon interface. The latter is guaranteed by the geometry of a thin film on a thick substrate. In contrast, the absence of any significant surface dissipation in the metal films is unexpected and 
worth highlighting. Experiments on single-crystal silicon cantilevers, with thickness ranging from tens of nanometers to a few microns, have frequently identified surface dissipation as a major source of damping [Yas00, Yan00], but the specific mechanisms responsible for energy loss at the surface have not yet been identified.

It is difficult to make a detailed quantitative comparison of the results on internal friction here with those reported earlier in the literature because of crucial differences in technique, test conditions, and geometry. Previous studies have focused mostly on exploring the effects of temperature on internal friction at a few values of thickness and frequency. Moreover, these studies have focused typically on films with thickness on the order of $1 \mu \mathrm{m}$, in contrast to the measurements here (on thinner films, 50 to $500 \mathrm{~nm}$ ). The measurements here are from beams vibrating in the fundamental mode; higher frequencies have not been accessed by exciting higher modes of vibration, as is often the case in many other studies. Finally, the approach taken in this study relies upon the use of thermoelastic damping for calibration, and an explicit mechanism-by-mechanism partitioning of the dissipated energy. In contrast, all previous studies have used a layer-by-layer partitioning following Equation (2.3) or Equation (2.4), and have not accounted for thermoelastic damping in the composite.

Nevertheless, we can attempt a qualitative comparison with some earlier studies. Liu et al [Liu99] measured damping in thin films deposited on silicon by using double-paddle torsional oscillators vibrating at $5.5 \mathrm{kHz}$. The internal friction in evaporated films of gold with thickness of $10 \mathrm{~nm}, 31 \mathrm{~nm}, 106 \mathrm{~nm}$, and $310 \mathrm{~nm}$ did not change significantly with thickness at temperatures ranging from 0.5 to $100 \mathrm{~K}$. The results in our study lead to the same conclusion for gold films at room temperature. In contrast, internal friction in Al and Ag increased significantly when the thickness was reduced below $100 \mathrm{~nm}$. The second comparison is with a set of studies of internal friction in aluminum films at high temperatures [Ber81, Pri94, Cho04]. The internal friction consists of a temperature-dependent 
background (presumably due to dislocation activity) and a peak attributed to grain-boundary sliding. It is therefore possible that both these mechanisms contribute to the internal friction measured in the thin Al films in this study. However, the relative dominance of these two mechanisms at room temperature is not currently known. Finally, it is instructive to compare our measurements in this study with some early work on damping in bulk metals. Routbort and Sack [Rou66] measured damping in bulk metallic wires (with a diameter of $1 \mathrm{~mm}$ ) oscillating in torsion at room temperature, and found that internal friction in Al stayed essentially constant as the frequency was increased from $1 \mathrm{~Hz}$ to $40 \mathrm{kHz}$. In contrast, in this study, internal friction in $\mathrm{Al}$ films reduced when frequency was increased from $100 \mathrm{~Hz}$ to $1.5 \mathrm{kHz}$. Measurements over a wider range of frequencies are necessary to confirm this trend. This observation motivates the work presented in Chapter 4 to characterize the frequency dependence of internal friction in thin $\mathrm{Al}$ films.

The results presented in this chapter are the first measurements of the effects of thickness and frequency on internal friction at room temperature in thin films of aluminum, silver, and gold. These results provide some useful guidelines for designing layered resonators for MEMS: gold leads to a smaller increase in damping than either aluminum or silver, and damping in the composite resonators can be decreased by reducing the film thickness.

\subsection{Summary}

This chapter described the first application of the new approach to measuring internal friction using the first generation of the silicon microcantilever platform. Using this approach, the internal friction in thin films of polycrystalline aluminum, silver and gold was measured. Measurements were carried out over a frequency range from 97.5 to $1445 \mathrm{~Hz}$ and over a thickness range from 60 to 450 $\mathrm{nm}$. In all cases, we see that the internal friction is due to processes occurring in the film and not at the film/silicon interface or the free surface of the metal. The internal friction in aluminum is consistently higher than the internal friction in 
gold for the entire range of thicknesses. Therefore, gold is a better choice than Al for metalizing high- $Q$ ceramic microcantilevers. Similarly, reducing the film thickness to the extent possible for the specific application under consideration will also reduce dissipation. These are the first two design guidelines that emerge from studies conducted using the silicon microcantilever platform. 


\section{Chapter 4}

\section{Measurement and Analysis of Internal Friction in Thin Films of Aluminum over Three Decades of Frequency}

\section{Introduction}

Accurately predicting the response of micro or nano-resonators at a given frequency is critically dependant on knowing the damping in the system. With clamping, support and internal friction losses minimized, we have seen that the response of single-crystal silicon cantilevers can be accurately characterized by Zener's model of TED. Therefore, with the setup described in Chapter 2, at a given frequency, the damping in a single-crystal resonator can be predicted to better than $10^{-5}$. With the addition of a thin film, we have seen that there is a disproportionate increase in damping. Evidently, the contribution from the thin film to TED in the composite is negligible $\left(h_{f} \leq 10^{-3} h_{s}\right)$ and can be ruled out as a major contributor to the observed increase. This raises the questions: what mechanisms account for the increased damping; and does this behavior fit existing models of material damping?

Vital to answering these questions is the ability to accurately measure dissipation in these thin films over a wider range of frequencies. In this chapter, we examine the use of the microcantilever platform to measure and analyze the dissipation in $60 \mathrm{~nm}$ thick films of aluminum over three decades of frequency. One of the challenges with such a measurement is the ability to maintain an adequate resolution over the entire frequency range. In section 4.1, the minimum resolution of a vibrating reed setup is discussed and a strategy to maintain adequate resolution by controlling specimen thickness is presented. Section 4.2 details the fabrication of high frequency $(>1.5 \mathrm{kHz})$ beams. Subsequently, section 4.3 presents the results of measurements of damping and internal friction in the films over frequencies from $70 \mathrm{~Hz}$ to $40 \mathrm{kHz}$. To conclude, the results are compared with the few available studies in literature. 


\subsection{Resolution of measurement for internal friction in thin films using the vibrating reed approach}

One of the advantages of the vibrating reed technique is the ability to use a single specimen to measure damping over a range of frequencies. This is accomplished by exciting higher frequency modes of the beam. Indeed, vibrations over eight vibration modes (or 2 decades of frequency, $35 \mathrm{~Hz}-4 \mathrm{kHz}$ ) have been used to obtain internal friction measurements of $100 \mathrm{~nm}$ thick Al films [Ber75]. Alternately, several researchers have utilized specimens with a large tip mass attached at the end of silicon cantilevers [Pri94, Boh92, Cho04]. By adjusting the mass at the end of the cantilever they were able to study damping over a range of frequencies ( 1 decade of frequency). Crucially, both these approaches use a beam of constant thickness to measure damping over a wide frequency range. However, for the setup in this thesis wherein the beams approach the limits of TED, using a cantilever of a single thickness over the entire frequency range can lead to a loss in measurement resolution. To illustrate this point, let us first examine the measurement resolution of a typical vibrating reed measurement system.

\subsubsection{Calculating the resolution of measurement}

Here, we refer to the resolution as the smallest change in damping that can be measured accurately. A measurement of the damping in the bare silicon substrate, $\delta_{s}$, has certain errors that arise from manually clamping the beam in the setup. A measurement of damping in the substrate is therefore [Tay82]

$$
\delta_{s}(1 \pm \varepsilon),
$$

where $\varepsilon$ is the relative error in the measurement of the substrate. In a similar manner the measured damping in the film/substrate composite is

$$
\delta_{c}(1 \pm \varepsilon)
$$


For simplicity, we ignore process variations in the film and consider only errors in the setup. From the repeatability tests in described in section 2.5, the maximum relative error in a measurement was $8 \%$. To be considered as a valid measurement, the minimum absolute damping in the composite must be equal to or greater than the maximum absolute error in the measurement of damping in the substrate:

$$
\delta_{c, \min }(1-\varepsilon) \geq \delta_{s}(1+\varepsilon) .
$$

The smallest value of composite damping that can be accurately measured can be obtained by solving Equation 4.3 for $\delta_{c, \text { min }}$ to obtain

$$
\delta_{c, \min } \geq \delta_{s} \frac{(1+\varepsilon)}{(1-\varepsilon)} .
$$

For small values of $\varepsilon$, Equation 4.4 can be simplified to obtain

$$
\delta_{c, \min } \geq \delta_{s}(1+2 \varepsilon) \text { for } \varepsilon<<1 \text {. }
$$

The minimum measurable change in damping is given by

$$
\left(\delta_{c}-\delta_{s}\right)_{\min } \geq 2 \varepsilon \delta_{s},
$$

and can be scaled by Equation (2.10) to obtain the resolution of a measurement of internal friction in the film:

$$
\left(\delta_{f}\right)_{\min } \geq 2 \varepsilon \delta_{s} \frac{E_{s} h_{s}}{3 E_{f} h_{f}} .
$$

From Equation (4.7), we see that for thin films on a thick substrate, a lower substrate damping and a lower $h_{s} / h_{f}$ are the biggest contributors to higher resolution. Equation (4.7) is similar to the resolution estimate provided by Berry and Pritchet [Ber75] and differs only by the inclusion of the error in repeatability. In their analysis, they select a minimum criterion of $\delta_{c, \text { min }} \approx \delta_{s}$; an error equivalent to double the measured damping value. 
With this resolution criterion, let us examine an internal friction measurement of a $100 \mathrm{~nm}$ thick aluminum film as a function of frequency using four single-crystal silicon beams. The beams all have a thickness of $100 \mu \mathrm{m}$ and the length is adjusted such that the beams have a first mode resonance that ranges from $100 \mathrm{~Hz}$ to $3500 \mathrm{~Hz}$. Assuming that viscous, clamping and support losses have been sufficiently reduced, the uncoated beam should operate close to the TED ( $\delta_{s} \approx \delta_{s, T E D}$ ) limit for all modes. We can, therefore predict the damping in each of the beams using Zener's expression (Equation (2.6)) and calculate the resulting resolution from Equation (4.7).

The results of the calculation are shown in Table 4.1. Based on this beam geometry, higher operating frequencies have higher damping due to TED. As a result, the resolution of an internal friction measurement in a $100 \mathrm{~nm}$ thick Al film drops from $10^{-4}$ at $100 \mathrm{~Hz}$ to $10^{-2}$ at $3500 \mathrm{~Hz}$ using these beams. This resolution at higher frequencies is of the order of the internal friction measurements carried out in Chapter 3 ( $\delta_{f} \approx 0.06$ at $100 \mathrm{~Hz}$ ) and is therefore undesireable.

Table 4.1: Predicted minimum resolution in four $100 \mu \mathrm{m}$ thick silicon cantilevers used to measure internal friction in a $100 \mathrm{~nm}$ thick Al film as a function of frequency. All beams are assumed to operate at the TED limit.

\begin{tabular}{ccc}
\hline $\begin{array}{c}\text { Frequency } \\
(\mathbf{H z})\end{array}$ & Predicted Damping & Predicted resolution \\
& $\delta_{s} \approx \delta_{s, T E D}^{E q n, 2.7}$ & $\left(\delta_{f}\right)_{\min }^{E q n, 4.7}$ \\
& $\times 10^{-5}$ & \\
\hline 100 & 4.62 & 0.00058 \\
600 & 2.89 & 0.0033 \\
1750 & 7.99 & 0.0090 \\
3500 & 15.0 & 0.0169 \\
\hline
\end{tabular}




\subsubsection{Maintaining a high resolution over a wide range of frequency}

A high resolution can be maintained by ensuring a low enough substrate damping over the desired frequency range and by decreasing $\left(h_{s} / h_{f}\right)$. For a setup wherein the silicon beams operate close to the TED limit, the damping can be tailored to be low at any specific frequency by appropriately selecting the thickness of the beam. In fact, to maintain resolution over a wide range of frequency, a simple design based approach would be to first select a maximum damping level in the beam $\left(\delta_{s}\right)_{\max }$ and then select the appropriate beam geometry for a specific frequency. From equation (2.6), when the beams operate close to TED

$$
\left(\delta_{s}\right)_{\max } \approx\left(\delta_{s, T E D}\right)_{\max }=\frac{\pi E_{S} \alpha_{s}^{2} T_{0}}{C_{s}} \frac{\Omega_{\max }}{1+\Omega_{\max }^{2}}=\Delta \frac{\Omega_{\max }}{1+\Omega_{\max }^{2}},
$$

which can be solved for the maximum allowable normalized frequency, $\Omega_{\max }$, to obtain

$$
\Omega_{\max }=\frac{\Delta}{2\left(\delta_{s, T E D}\right)_{\max }} \pm \sqrt{\left(\frac{\Delta}{2\left(\delta_{s, T E D}\right)_{\max }}\right)^{2}-1}
$$

Here we only select values for $\Omega_{\max }<1$ and given that

$$
\Omega_{\max }=\omega \frac{C_{s} h_{s, \max }^{2}}{\pi^{2} k_{s}}
$$

we see that for any specific frequency, $\omega$, we can determine a maximum beam thickness, $h_{s, \max }$ below which $\left(\delta_{s}\right)_{\max }$ is not exceeded. The frequency of the beam can be controlled by adjusting the length at the calculated thickness.

This approach can be summarized in four simple steps:

i) Select the desired upper limit on damping in the substrate $\left(\delta_{s}\right)_{\max }$.

ii) Calculate the upper limit on the normalized frequency $\left(\Omega_{\max }\right)$ using Equation (4.8). 
iii) Calculate the maximum allowable beam thickness $\left(h_{s, \max }\right)$, at the desired frequency from Equation (4.10).

iv) Adjust the length of the beam to obtain the appropriate resonant frequencies.

The next section utilizes this approach to measure the internal friction in $60 \mathrm{~nm}$ thick films of aluminum over a frequency range from $70 \mathrm{~Hz}$ to $40 \mathrm{kHz}$.

\subsection{Design, fabrication and measurement of high frequency single-crystal silicon beams}

\subsubsection{Design requirements for the beams}

The design requirement was stated as the ability to measure internal friction in $\sim 60 \mathrm{~nm}$ films of $\mathrm{Al}$ with a resolution better than 0.003 over a frequency range of $70 \mathrm{~Hz}-100 \mathrm{kHz}$. The resolution was selected to be $1 / 10^{\text {th }}$ of the maximum measured internal friction in these films from Section (3.4). The maximum

acceptable value, $\left(\delta_{s}\right)_{\max }$, for the damping in the uncoated single-crystal silicon beams was selected to be $3 \times 10^{-5}$ over the entire range of frequency.

To determine the appropriate beam thicknesses, the frequency range was first divided into three groups: $70 \mathrm{~Hz}-1 \mathrm{kHz}, 1-10 \mathrm{kHz}$, and 10-100 kHz. The procedure outlined in section 4.1.2 was then used to estimate $h_{s, \max }$ such that the damping in the beam would not exceed $\left(\delta_{s}\right)_{\max }$ at the highest frequency in each range. The results showed that beams that were less than $80 \mu \mathrm{m}, 24 \mu \mathrm{m}$ and $8 \mu \mathrm{m}$ could be used for measurements below $1 \mathrm{kHz}, 10 \mathrm{kHz}$ and $100 \mathrm{kHz}$, respectively. These threshold thicknesses were, therefore, used as guidelines for the fabrication and use of beams over the four decades of frequency.

Evidently, using beams that were $8 \mu \mathrm{m}$ in thickness would provide adequate resolution over a frequency range from $70 \mathrm{~Hz}$ to $40 \mathrm{kHz}$. However, the low 
frequency beams $(<1 \mathrm{kHz})$ have high aspect ratios $\left(L / h_{s}\right)$ that make handling and fabrication of the beams difficult. For example, a $300 \mathrm{~Hz}$ beam that is $8 \mu \mathrm{m}$ thick has an aspect ratio of $L / h_{s}=600$. Trying to manually handle such a beam would be difficult. Therefore, thicker beams were preferred for higher aspect ratios $\left(L / h_{s}>100\right)$. The next section describes the fabrication of cantilevers for these measurements.

\subsubsection{Fabrication of the cantilevers}

Single-crystal silicon beams for frequencies up to $1500 \mathrm{~Hz}$ were fabricated as outlined in section 2.3. The final step in this fabrication process involves manually breaking the beam at the appropriate length to obtain the desired frequency. This approach could not be used for the smaller cantilevers (thinner and shorter) required for measurements at higher frequencies. Therefore, an alternate process had to be developed to fabricate beams with frequencies higher than $1.5 \mathrm{kHz}$.

This alternate process makes use of commercially available silicon on insulator (SOI) wafers. Typically, an SOI wafer is produced by bonding two polished silicon wafers with a thin oxide layer sandwiched in between (Figure 4.1). One of the wafers is then thinned down to the desired thickness by chemical mechanical polishing [Ton99]. This thinned down wafer is commonly termed the device layer and can be used to produce silicon structures with a very precise thickness. In our case, the device layer forms the cantilevered beam while the other bonded wafer forms the base.

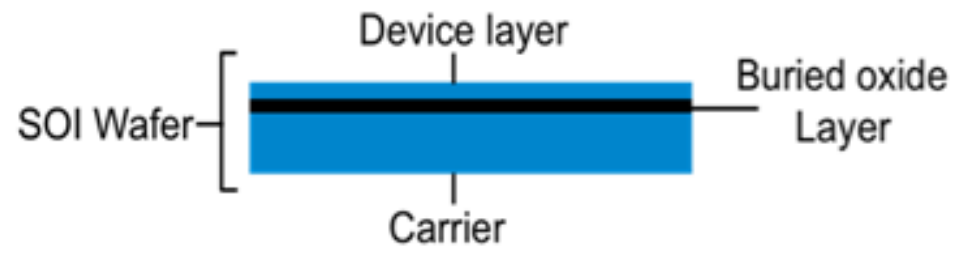

Figure 4.1: Schematic of two single crystal silicon wafers that are bonded to form a silicon on insulator (SOI) wafer. 
Table 4.2 shows the process flow for the fabrication of these high frequency beams. The starting materials for the beams were $\mathrm{p}$-type, single-crystal silicon, six inch, SOI wafers with device layers that were $24 \mu \mathrm{m}$ and $8 \mu \mathrm{m}$ in thickness. In both cases, the carrier wafers were between 500 and $600 \mu \mathrm{m}$ thick. These wafers are doped with boron at a low concentration of $\sim 10^{15} \mathrm{~cm}^{-3}$ (Table 4.2a). First, the wafers were oxidized for 26 minutes at $1100{ }^{\circ} \mathrm{C}$ to grow $\mathrm{SiO}_{2}$ films with a thickness of $\sim 500 \mathrm{~nm}$ on both surfaces (Table $4.2 \mathrm{~b}$ ). The oxide films on the device layer were then patterned using a standard photolithographic process and the silicon dioxide etched by reactive-ion etching to expose the underlying silicon (Table 4.2c). The wafers were then recoated with an $11 \mu \mathrm{m}$ thick layer of a photoresist AZ9245 (AZ Electronic Materials, USA), which is a highly selective mask for deep reactive ion etching (DRIE) of silicon. The resist was then patterned to match the existing silicon dioxide (Table 4.2d) and the exposed silicon was subsequently etched by DRIE in a CHF3:CF4:Ar (40:1:70) gas plasma. The buried oxide layer was used as the etch stop.

The wafers were then cleaned and once again oxidized for 4 minutes at $1100{ }^{\circ} \mathrm{C}$ to grow a $100 \mathrm{~nm} \mathrm{SiO}$ film on the etched structures (Table 4.2e). This oxide layer serves as a protective mask for the beam in subsequent DRIE and TMAH etch steps. The oxide on the backside of the wafer was then patterned to define the rectangular base of the cantilever and the exposed silicon etched over $500 \mu \mathrm{m}$ to the buried oxide layer (through the thickness of the carrier wafer) (Table 4.2f). This process was not finely tuned and resulted in etch rate differences across the wafer. That is, some areas were etched down to the buried oxide layer faster than other regions. Therefore, after the DRIE step, the wafers were placed in a $27 \%$ TMAH solution at $85{ }^{\circ} \mathrm{C}$ until all the exposed silicon was etched away (Table $4.2 \mathrm{~g})$. The beams were then carefully extracted from the wafer and etched in concentrated hydrofluoric acid $\left(1: 1, \mathrm{HF}: \mathrm{H}_{2} \mathrm{O}\right)$ for 5 minutes to remove the oxide surrounding the cantilever and base (Table 4.2h). An image of a finished cantilever is shown in Figure 4.2 showing the difference in the base structure in comparison to the lower frequency beams shown in section 2.2. 
Table 4.2: Process flow for fabrication of high frequency ( $>1500 \mathrm{~Hz}$ ) single crystal silicon beams starting with an silicon-on-insulator (SOI) wafer.

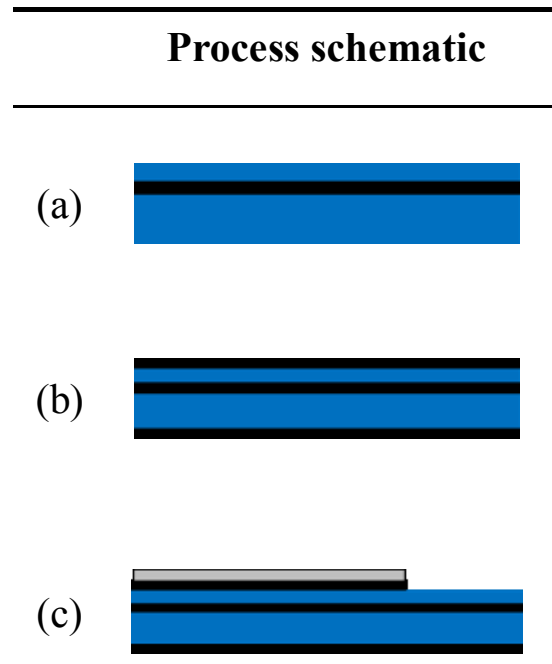

Starting material: Single crystal Si $(1,0,0)$, siliconon-insulator (SOI) wafers. 6" P-type lightly doped wafer layer with $\sim 0.5-2 \mu \mathrm{m}$ thick buried oxide layer.

$500 \mathrm{~nm}$ of thermal oxide $\left(\mathrm{SiO}_{2}\right)$ was grown on either side to act as mask for etching.

Photolithography was carried out to pattern the cantilevers. The exposed oxide was etched away by reactive-ion-etching (RIE) using a CHF3:CF4:Ar (40:1:70) gas plasma (Applied Materials P5000 RIE).

The wafer was re-patterned with AZ9245 photoresist for subsequent Deep-reactive-ionetching (DRIE). The exposed silicon was then etched away by DRIE using $\mathrm{SF}_{6}: \mathrm{C}_{4} \mathrm{~F}_{8}: \mathrm{O}_{2}$ pulsed gasses and a source power 2700W (Tegal SDE110 DRIE).

(e)

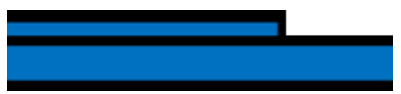

$100 \mathrm{~nm}$ of oxide was then grown on the etched structures as mask for subsequent wet etching.

Photolithographic patterning was carried out on the backside of the wafer and the exposed oxide was then etched away by RIE with process similar to step (c).

DRIE was used to etch through the device layer. Due to a non uniformity in etch rates across the wafer, the final etch was carried out in a $27 \%$ TMAH solution at $85^{\circ} \mathrm{C}$ until all the exposed silicon was etched away.

Each beam was then individually released from the wafer and placed in 1:1 HF: $\mathrm{H}_{2} \mathrm{O}$ for 5 minutes to etch away the oxide. The buried oxide layer was relatively unaffected.

- $\mathrm{SiO}_{2}$. $\square \mathrm{Si}$ Photoresist $\square$ AZ9245 DRIE photoresist 


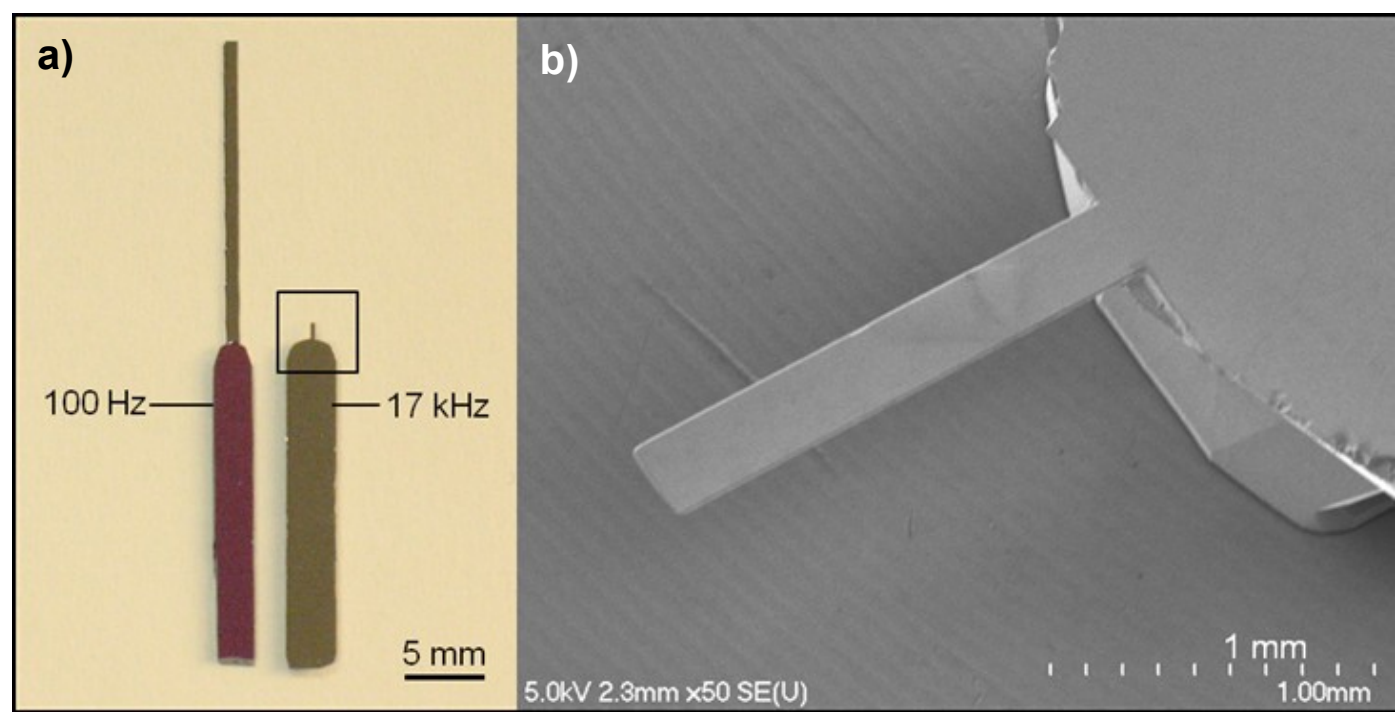

Figure 4.2: Images of beams used in this study: a) the larger, low frequency beam was fabricated with the process outlined in section 2.4 while the shorter, thinner and higher frequency $(\sim 17 \mathrm{kHz})$ beam is fabricated from SOI wafers as shown in section 4.3.2. b) SEM micrograph of a typical high frequency beam fabricated with this process.

\subsection{Measurement of damping in thin films of aluminum}

For this study, nine sets of beams were selected to span the range of frequencies from $72 \mathrm{~Hz}$ to $40.1 \mathrm{kHz}$. Each set consisted of two beams with nominally similar frequencies $(<20 \%$ difference). The damping in each of these beams was first measured by the procedure outlined in section 2.4 .

The beams were then coated with $\sim 60 \mathrm{~nm}$ of aluminum (with a $\sim 15 \mathrm{~nm}$ chromium adhesion layer) under the same conditions used previously (section 3.3). Two additional beams were coated with only the adhesion layer. Sputtering was carried out in groups of four beams that were randomly selected from the entire set. The damping in the composites was then measured to determine the effect of the film.

\subsubsection{Results}

The results of measurements of the twenty single-crystal silicon beams are shown in Table 4.3. From the results, we see that the first mode natural frequencies of the beams differ from the predicted Euler Bernoulli by a maximum of $\pm 10 \%$ but with standard deviation of $\pm 2 \%$. Also shown in the table is the predicted 
resolution, $\left(\delta_{f}\right)_{\min }^{E q 4.7}$. As a result of the low beam thickness used at low frequencies, the beams have a substantially higher resolution than the initial design requirements.

Table 4.3: Measurements of damping in the 20 single-crystal silicon cantilevers used in this study. Also shown is the predicted and actual resolution of the beam when measuring a $60 \mathrm{~nm} \mathrm{Al}$ film as calculated in Equation (4.7)

\begin{tabular}{|c|c|c|c|c|c|c|c|}
\hline $\begin{array}{c}h_{s} \\
(\mu \mathbf{m})\end{array}$ & $\begin{array}{c}\text { Length } \\
(\mathrm{mm})\end{array}$ & $\begin{array}{c}\text { Predicted } \\
1^{\text {st }} \text { Mode } \\
\text { frequency } \\
(\mathbf{H z})\end{array}$ & $\begin{array}{c}\text { Measured } \\
1^{\text {st }} \text { mode } \\
\text { frequency } \\
(\mathrm{Hz})\end{array}$ & $\begin{array}{l}\delta_{S, T E D} \\
\times 10^{-6}\end{array}$ & $\begin{array}{c}\delta_{s} \\
\times 10^{-6}\end{array}$ & $\begin{array}{c}\left(\delta_{f}\right)_{\min }^{E q 4.7} \\
\times 10^{-4}\end{array}$ & $\left(\delta_{f}\right)_{\min }$ \\
\hline 97 & 42.0 & 76.07 & 72.06 & 3.12 & 13.8 & 5.0 & 0.0025 \\
\hline 83 & 40.0 & 71.76 & 79.44 & 2.52 & 12.9 & $3.9 \mathrm{E}-04$ & 0.0020 \\
\hline 28 & 19.0 & 107.30 & 107.49 & 0.38 & 9.87 & 0.24 & 0.0005 \\
\hline 24 & 17.0 & 114.88 & 118.11 & 0.31 & 7.94 & 0.14 & 0.0004 \\
\hline 26 & 10.0 & 359.69 & 355.73 & 1.11 & 6.80 & 0.54 & 0.0003 \\
\hline 26 & 10.0 & 366.45 & 360.02 & 1.12 & 7.15 & 0.54 & 0.0003 \\
\hline 26 & 7.50 & 639.44 & 638.29 & 1.98 & 8.70 & 0.96 & 0.0004 \\
\hline 28 & 7.70 & 653.32 & 655.68 & 2.36 & 9.76 & 1.2 & 0.0005 \\
\hline 28 & 7.50 & 688.63 & 700.73 & 2.53 & 8.57 & 1.3 & 0.0005 \\
\hline 27 & 5.50 & 1234.78 & 1262.80 & 4.23 & 16.2 & 2.1 & 0.0008 \\
\hline 26 & 5.10 & 1382.87 & 1395.73 & 4.34 & 9.64 & 2.1 & 0.0005 \\
\hline 23 & 3.58 & 2482.63 & 2459.33 & 5.98 & 10.3 & 2.5 & 0.0004 \\
\hline 23 & 3.58 & 2482.63 & 2488.00 & 6.05 & 10.3 & 2.6 & 0.0004 \\
\hline 23 & 2.35 & 5552.24 & 5552.24 & 13.5 & 19.0 & 5.8 & 0.0008 \\
\hline 23 & $2 . .35$ & 5761.59 & 5681.67 & 13.8 & 20.3 & 5.9 & 0.0009 \\
\hline 7.5 & 0.80 & 16211.80 & 15333.60 & 3.9 & 13.0 & 0.55 & 0.0002 \\
\hline 7.5 & 0.80 & 16211.80 & 16536.80 & 4.28 & 11.6 & 0.60 & 0.0002 \\
\hline 8.0 & 0.80 & 17292.59 & 17095.20 & 5.03 & 13.2 & 0.78 & 0.0002 \\
\hline 7.5 & 0.49 & 43213.46 & 38880.80 & 10.1 & 19.2 & 1.40 & 0.0003 \\
\hline 7.5 & 0.49 & 43213.46 & 40118.00 & 10.4 & 16.7 & 1.40 & 0.0002 \\
\hline
\end{tabular}


Let us now turn to the damping measurements. Figure 4.3 shows a plot of the logarithmic decrement in the single-crystal silicon beams, the predicted TED and the damping in the composite for the beams as a function of frequency. All measurements were for beams operating in the first resonant mode. The predicted damping curve shows abrupt decreases at $100 \mathrm{~Hz}$ and $16 \mathrm{kHz}$ due to the difference in the thickness of the beams used between these frequencies: the beams range in thickness from 23-28 $\mu \mathrm{m}$ for measurements between $100 \mathrm{~Hz}$ and $5 \mathrm{kHz}$ and from 7.5-8 $\mu \mathrm{m}$ above $5 \mathrm{kHz}$. In comparison, the actual measured damping in the beams follows the same trend with a relatively constant offset.

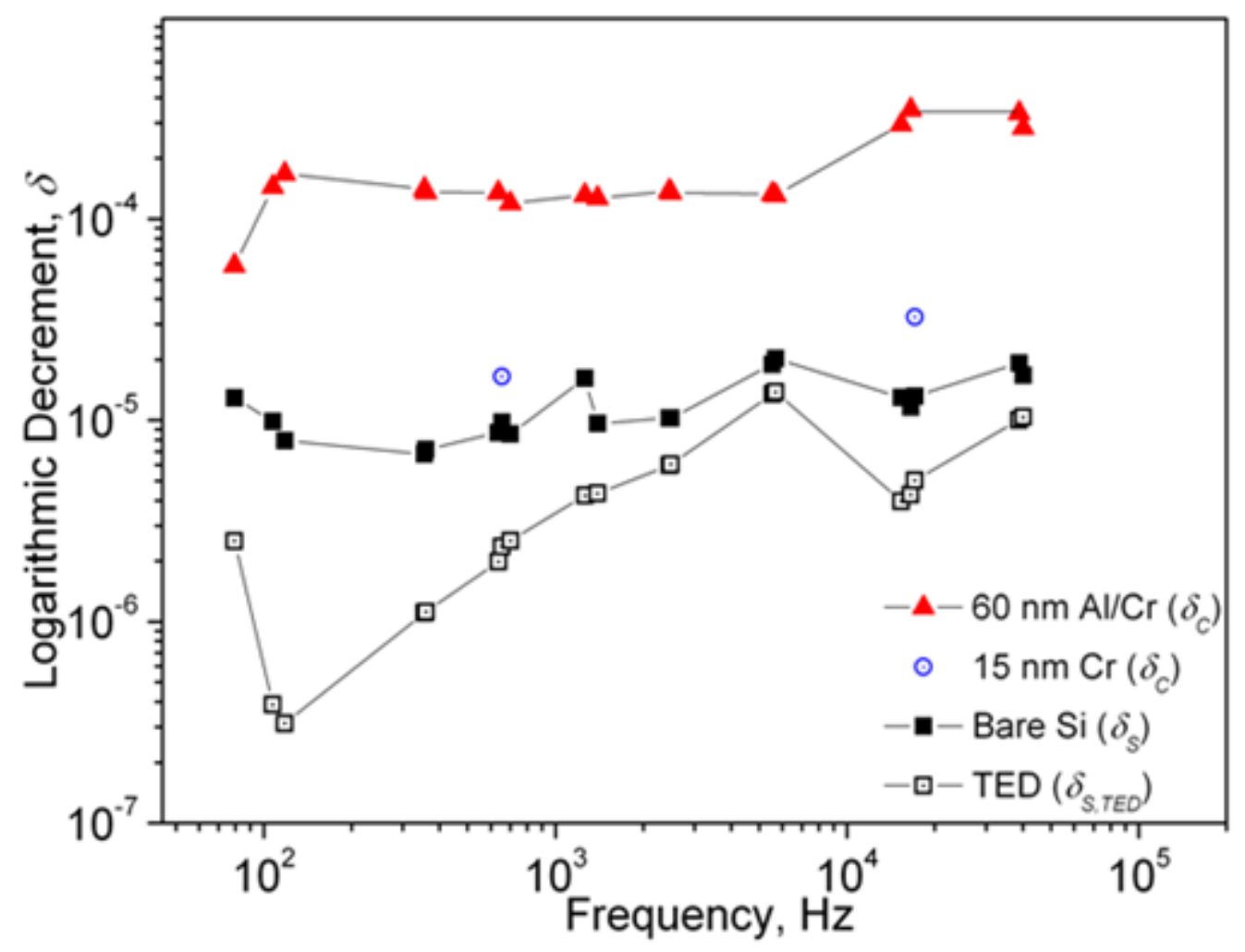

Figure 4.3: Frequency dependence of the damping in the beams used in this study. Shown is predicted TED in the beams using Zener's formula, the measured damping in the beams, the change with a $15 \mathrm{~nm}$ adhesion layer and the measured damping after coating with $60 \mathrm{~nm}$ of $\mathrm{Al}$ (with $15 \mathrm{~nm}$ adhesion layer). 
Turning our attention to the damping in the composite, we see that for the two beams coated with $15 \mathrm{~nm}$ of Cr only, there is a measurable change in damping in the order of $1 \times 10^{-5}$. In contrast to the measurements in the study in Chapter 3, these beams had a higher resolution (thinner and with lower damping) and could be used to discern the damping contribution from these ultrathin layers of chromium.

From Figure 4.3 we see that the addition of aluminum increases the damping in the structures by over an order of magnitude. The damping in the composite increases proportional to the volume fraction of the film in the structure as can be seen from increased composite damping in beams operating between $100-300$ $\mathrm{Hz}$ and above $5 \mathrm{kHz}$.

We can extract the internal friction in the film over the three decades of frequency from the measured data. Figure 4.4 shows the internal friction in the Al film calculated from Equation (2.10) for all the beams used in this study. In contrast to the damping in the composite, we see that the damping in the film decreases monotonously over the measured range from a value of 0.053 to 0.028 . The variation in measurements is consistent with the $15 \%$ variation in thickness measurements between each deposition run. 


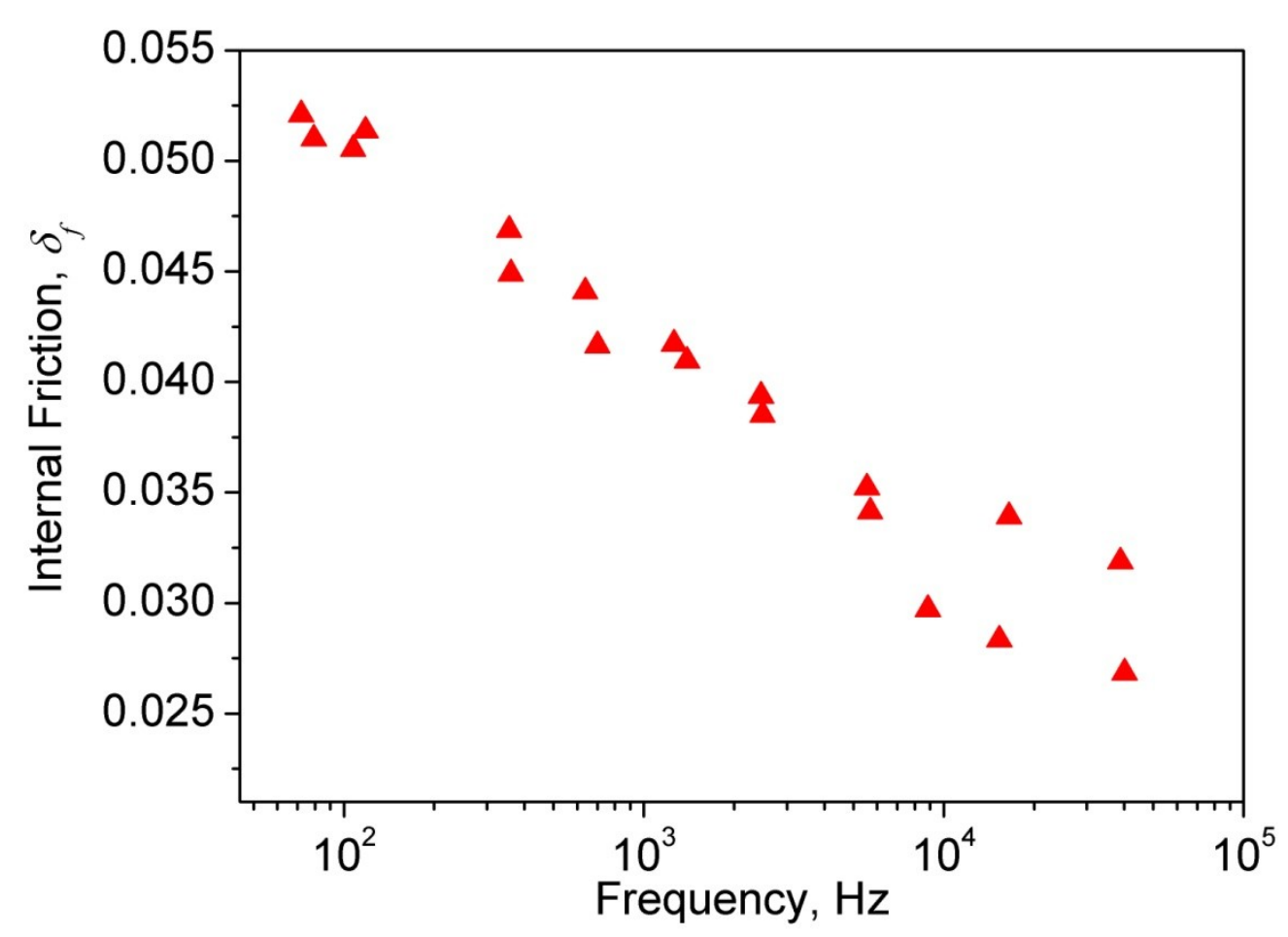

Figure 4.4: Frequency dependence of internal friction in $60 \mathrm{~nm}$ thick films of aluminum.

\subsubsection{Discussion}

Let us first begin with the results in Table 4.3 and in Figure 4.3. The volume fractions of the $\mathrm{Al}$ or $\mathrm{Cr}$ films in the composite are still in the order of $10^{-3}$ $\left(h_{f} / h_{S} \approx O\left(10^{-3}\right)\right)$ and therefore $\delta_{C, T E D} \cong \delta_{S, T E D}$ for all the measurements. The measured damping in the uncoated cantilevers remains relatively constant over frequency and the trend of the predicted TED for damping values greater than $6 \times 10^{-6}$. If we compare the measured damping in the substrate with the predicted TED, we see that the residual damping $\left(\delta_{S}-\delta_{S, T E D}\right)$ varies between $3 \times 10^{-6}$ and $1 \times 10^{-5}$. This is consistent with the clamping losses associated with the setup as discussed in section 2.5. However, even with this residual damping, all the cantilevers have sufficient measurement resolution ranging from 0.002 at $70 \mathrm{~Hz}$ to 0.0002 at $40 \mathrm{kHz}$ for a $60 \mathrm{~nm}$ thick film of aluminum. 
Turning our attention to the adhesion layers, the decreased substrate thickness and lower initial damping implies that the effect of the $15 \mathrm{~nm}$ adhesion layer is clearly discernible. The damping due to the adhesion layer was measured for beams at two different thicknesses $(28 \mu \mathrm{m}$ and $8 \mu \mathrm{m})$ and at two different frequencies to analyze the effect of both variables. In both cases, we see that although the adhesion layer accounts for approximately $1 / 5^{\text {th }}$ of the thickness of the $\mathrm{Al} / \mathrm{Cr}$ film it accounts for less than $5 \%$ of the measured damping in the $\mathrm{Al} / \mathrm{Cr}$ film. This clearly indicates that the measured effect is largely due to the addition of the aluminum. It is also worth noting that these measurements highlight the ability of the approach in this study to measure the damping in ultra thin metal films $(<15$ $\mathrm{nm})$.

The results shown in figure 4.4 are the first measurements of internal friction in thin films of aluminum over three decades of frequency. The results show a monotonic twofold decrease $(\sim 0.053$ to $\sim 0.028)$ in the magnitude of internal friction over the entire range. These results extend the previous measurements in the study in Chapter 3 and show that even at $40 \mathrm{kHz}$ there is a significant amount of dissipation in $60 \mathrm{~nm}$ thick aluminum films. Measurements at these frequencies are immediately relevant to the broad class of cantilevered resonators used in atomic force microscopy measurements.

From the measurements of internal friction in $60 \mathrm{~nm}$ thick Al films, it is not clear what material damping mechanisms contribute to the observed frequency dependence. Previous measurements of $100 \mathrm{~nm}$ films of aluminum as a function of temperature have shown two distinct internal friction peaks [Ber75, Pri94, Cho04] superimposed on some amount of background damping. Both these peaks have been attributed to anelastic (recoverable) sliding of grain boundaries and the background damping due to the motion of dislocations in the thin film. Both these mechanisms can contribute to internal friction at room temperature. Further studies are required to understand the contributions of dislocations and grain boundaries to internal friction at room temperature in these thin aluminum films. 


\subsection{Summary}

Measurements of damping as a function of frequency are necessary for the design of micro- and nano-resonators. This chapter focused on a design based approach to measure the internal friction in thin films as a function of frequency. The impact of substrate damping on the precision of an internal friction measurement was discussed. Subsequently, a simple criterion for determining the maximum damping in the substrate to maintain a desired resolution of measurement was provided. This is an enhancement to the estimate provided by Berry and Pritchet [Ber75]. In this setup, the single-crystal silicon resonators operate close to TED. Therefore, Zener's expression (Equation (2.6)) can be used as a design guideline to fabricate beams that do not exceed the maximum damping criteria. Quite simply, this can be achieved by controlling the thickness of the beams for a given frequency.

This approach was then utilized to measure the internal friction in $60 \mathrm{~nm}$ thick films of aluminum over four decades of frequency. The results showed that the internal friction in aluminum drops by a factor of two over three decades of frequency. The lack of a significant change in damping as a function of frequency would suggest that a number of different mechanisms with different relaxation times are responsible for the damping. Lastly, from a design standpoint, there is still a significant amount of damping due to the films $(\sim 0.03)$ at $40 \mathrm{kHz}$. This study provides the first quantification of the effect of thin aluminum films over a frequency range applicable to widely used AFM cantilevers. 


\section{Chapter 5}

\section{Controlling Damping and $Q$ Factors of Silicon Microcantilevers by Selective Metallization}

\section{Overview}

The addition of thin polycrystalline metallic films on a silicon micro-resonator causes a disproportionate increase in dissipation. From a design standpoint, the lack of a comprehensive empirical database, accurate theoretical models, and reliable process-structure-dissipation correlations make it difficult to optimize the selection of materials and processes for different applications. Practical strategies for mitigating the adverse impact of metals on damping are therefore vital for improving the performance of composite micro and nanoscale resonators.

As discussed in Chapter 3, one straightforward strategy to reduce the dissipative impact of these films at room temperature is to minimize their volume fraction by using as thin a film as functionally possible. A second strategy is to utilize materials with lower internal friction to achieve the same functionality. For instance, the use of gold instead of aluminum films for reflective coatings on cantilevers can reduce the dissipation by a factor of three for devices operating between 0.1 and $1.5 \mathrm{kHz}$.

A third strategy for controlling dissipation is based on the rational selection of shape and geometry. This approach is based on two considerations. The first is the expectation that dissipation in the metal films is a function of their mechanical deformation during oscillation. The second is the observation that, for many applications, it is not necessary to coat the entire surface of the ceramic substrate with a metal. Taken together, these considerations suggest that selective metallization in regions of low strain can reduce dissipation and preserve functionality.

This strategy has been noted by several researchers; Sandberg et al proposed coating just the tip of silicon cantilevers with thin films of gold to minimize their 
dissipative impact [San05]. Later, Waggoner and Craighead experimentally demonstrated an increase in damping as a gold pad on a silicon cantilever was moved from the tip of the cantilever to the base [Wag09]. However, no model has been developed to accurately quantify and predict the effect of partial metallization on the damping in silicon cantilevers. Such a model would prove a valuable design tool not only for minimizing but also controlling the damping in a cantilevered beam.

In this chapter a simple model based on the distribution of strain energy along the length of the resonating beam is proposed. Section 5.1 outlines the development of an analytical model for analyzing the effects of patterning on damping. The predictions of this model are then systematically tested by comparison with measurements of damping in single-crystal silicon beams that are selectively metalized using thin aluminum films. The details of the experiments are presented in Section 5.2. Section 5.3 presents the principal results of this study: measurements of damping in uncoated silicon beams, change in damping after selective metallization, and a comparison of measurements with analytical predictions.

\subsection{Model for the effects of patterning on damping}

Consider a thin slender cantilevered beam of length $L$ and uniform solid rectangular cross section attached to a large base as shown schematically in Figure 5.1. The thickness and width of the beam are denoted $h_{s}$ and $w$, respectively. Patterned on this beam is a thin film of uniform thickness $h_{f}$ subject to the restriction that $h_{f}<<h_{s}$ and $h_{f}<<w$. The film spans the width of the beam and occupies the domain $x_{1}<x<x_{2}$, where $x$ is the coordinate along the axis of the beam with its origin at the root of the cantilever. The length of the pattern is much larger than the film thickness (i.e., $\left|x_{2}-x_{1}\right|>>h_{f}$ ). The adhesion between the film and substrate is assumed to be perfect. 


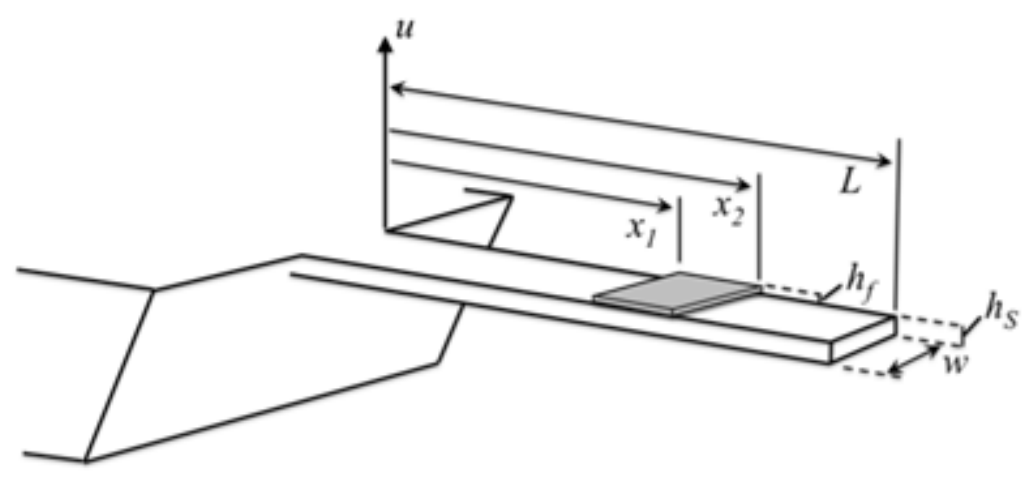

Figure 5.1: Schematic illustration of a microcantilever beam of length $L$ with a thin film coated over a portion of the top surface.

The dynamics of this composite structure can be analyzed by modeling it as an Euler-Bernoulli beam executing flexural vibration with small amplitude. In the limit of a thin film on a thick substrate $\left(h_{f}<<h_{s}\right)$, the mode shapes, natural frequencies, and location of the neutral axis of the monolithic beam are unaffected by the addition of the patterned thin film [Ber75, Sp107, Tam06, Pra05]. The structure vibrates in a vacuum chamber maintained at sufficiently low pressure so that viscous damping is negligible.

For the low values of dissipation under consideration, logarithmic decrement $(\delta)$ under free decay is related to the energy dissipated $\Delta W$ by [Gra92]

$$
\delta \approx \frac{\Delta W}{2 W_{\max }}
$$

The total damping in the composite structure can be expressed in terms of energy dissipated in the substrate and film [Ber75]. Hence,

$$
\delta_{c} \approx \frac{\Delta W_{c}}{2 W_{c, \text { max }}}=\frac{\Delta W_{s}+\Delta W_{f}}{2 W_{c, \text { max }}}
$$

In the limit of a thin film on a thick substrate, the contribution of the film to the denominator is negligible; that is, $W_{c, \max } \cong W_{s, \max }$. Therefore, Equation (5.2) can be expressed as [Ber75] 


$$
\delta_{c} \approx \frac{\Delta W_{s}+\Delta W_{f}}{2 W_{c, \text { max }}}=\frac{\Delta W_{s}}{2 W_{s, \text { max }}}+\left(\frac{W_{f, \text { max }}}{W_{s, \text { max }}}\right) \frac{\Delta W_{f}}{2 W_{f, \text { max }}}=\delta_{s}+\left(\frac{W_{f, \text { max }}}{W_{s, \text { max }}}\right) \delta_{f},
$$

where $\delta_{s}$ and $\delta_{f}$ are the logarithmic decrement of the bare substrate and patterned thin film, respectively. The term in the parenthesis is the ratio of the maximum stored elastic energy in the film and substrate.

Equation (5.3) highlights the two main strategies for controlling dissipation due to metallization: (i) select materials and processes to minimize internal friction in the film, and (ii) select the size and location of the pattern to minimize the strain energy in the film. The second strategy is the main focus of this chapter. To proceed, we evaluate the maximum strain energy using a standard analysis of the quasi-static deformation of an Euler-Bernoulli beam. For the substrate, we obtain

$$
W_{s, \max }=\frac{1}{2} \int_{0}^{1} E_{s} I_{s}\left(\frac{\partial^{2} \phi(\xi)}{\partial \xi^{2}}\right)^{2} d \xi
$$

where $\xi=(x / L)$ is the normalized length, $\varphi(\xi)$ is the natural mode shape (as explained in section 2.4), $E$ is the Young's modulus, $I$ is the area moment of inertia with $I_{s}=\frac{1}{12}\left(w h^{3}\right)$. Similarly, the maximum strain energy in the film is given by

$$
W_{f, \max }=\frac{1}{2} \int_{x_{1} / L}^{x_{2} / L} E_{f}\left[I_{f}+w h_{f}\left(\frac{h_{f}+h_{s}}{2}\right)^{2}\right]\left(\frac{\partial^{2} \phi(\xi)}{\partial \xi^{2}}\right)^{2} d \xi
$$

This expression assumes that the location of the neutral axis is unaffected by the presence of the patterned film because the geometry under consideration is that of a thin film on a thick substrate with $h_{f}<<h_{s}$.

Combining Equations (5.3), (5.4) and (5.5), and ignoring all terms of order $\left(h_{f} / h_{s}\right)^{2}$ and higher, we obtain 


$$
\delta_{c}=\delta_{s}+3 \frac{E_{f} h_{f}}{E_{s} h_{s}}\left[\frac{\left.\int_{x_{1} / L}^{x_{2} / L}\left(\frac{\partial^{2} \phi(\xi)}{\partial \xi^{2}}\right)^{2} d \xi\right]}{\int_{0}^{1}\left(\frac{\partial^{2} \phi(\xi)}{\partial \xi^{2}}\right)^{2} d \xi}\right] \delta_{f} .
$$

For the limiting case of a continuous thin film (that is, $x_{1}=0$ and $x_{2}=L$ ), the two integrals in Equation (5.6) are identical and this expression reduces to the formula derived by Berry \& Pritchet [Ber75]. This serves as a useful check on the derivation.

Equations (5.3) and (5.6) suggest that the metal must be patterned in locations of low strain energy. These optimal regions can be identified using a graph of the normalized strain energy as a function of location along the length of the beams. Figure 5.2 shows such a graph for the first mode of flexural vibrations of a cantilever. As an example, let us consider a metallic patch that is $20 \%$ the length of the beam. This patch will lead to significant dissipation if located at the root of the beam. In contrast, the same size of metal is expected to result in a negligible increase in dissipation if patterned at the free end of the beam. These observations motivate the experiments described in the next section. 


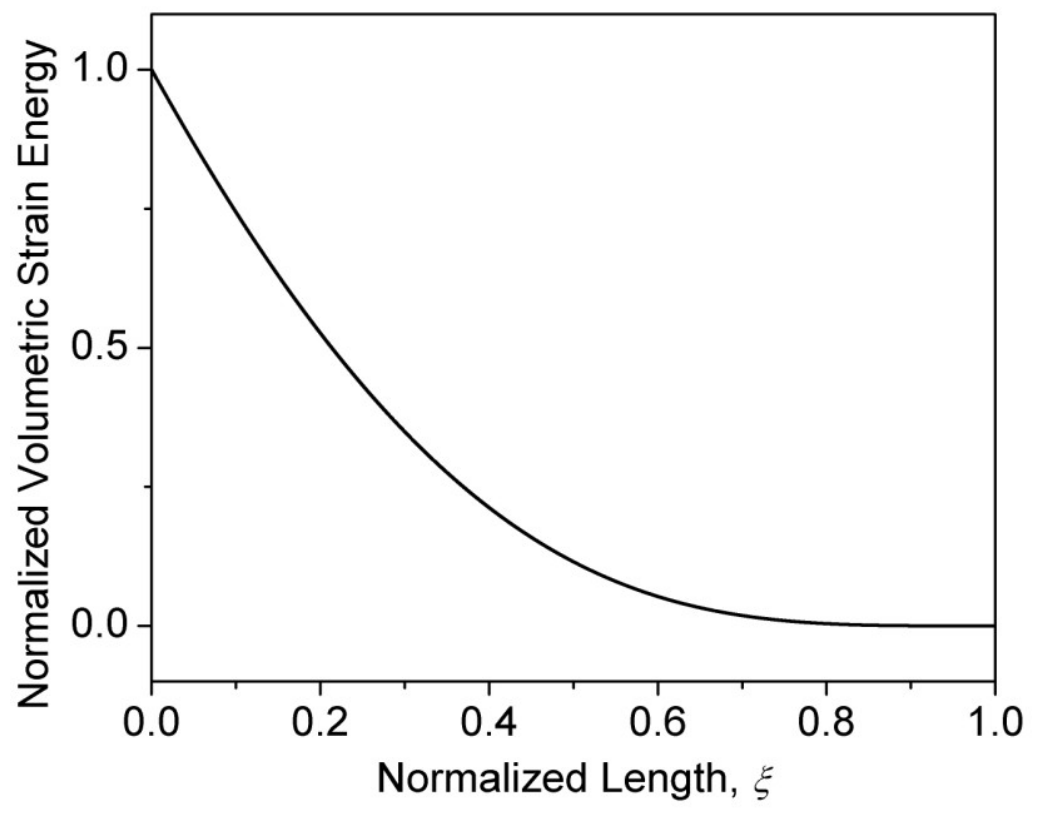

Figure 5.2: Volumetric strain energy distribution in the first mode of flexural vibration of a cantilevered Euler Bernoulli beam. The amplitude of vibration have been normalized to the maximum values of the strain energy.

\subsection{Experimental details}

This section describes the experimental work that was undertaken to confirm the predictions of the analytical models and derive guidelines for the design of high- $Q$ microresonators. For this study, silicon cantilevers were fabricated by the process outlined in section 2.3. A total of twelve cantilevered beams were produced with dimensions ranging from 78 to $85 \mu \mathrm{m}$ in thickness, 0.95 to $1.00 \mathrm{~mm}$ in width, and 22.5 to $24.6 \mathrm{~cm}$ in length. The variation in thickness along the length of the beams was less than $5 \%$. The fundamental natural frequencies of flexural vibrations of these beams ranged from $188 \mathrm{~Hz}$ to $233 \mathrm{~Hz}$.

\section{Selective metallization with aluminum films}

The silicon microcantilevers were divided into four sets (Set A-D) and selectively metalized with thin aluminum films. Each set contained three beams. The size and location of the metal patterns on each set are described in Table 5.1 by specifying 
their start and end locations along the axis of the beam. Patterning was achieved by lift-off using spray coating of $450 \mathrm{~nm}$ thick PMMA resist (EVG101 spraycoater, EV Group, NY, USA) and a shadow mask fabricated using strips of metal foil. All beams were metalized simultaneously by sputter deposition at room temperature using targets with purity greater than $99.99 \%$. Al films were then sputter deposited under the conditions described in section 3.2.2. In summary, the beams were first coated with a $10 \mathrm{~nm}$ thick $\mathrm{Cr}$ adhesion layer at a power density of $2.4 \mathrm{~W} / \mathrm{m}^{2}$ and subsequently with $100 \mathrm{~nm}$ of $\mathrm{Al}$ at a power density of $4.5 \mathrm{~W} / \mathrm{m}^{2}$ without breaking vacuum. Subsequently, a lift-off procedure was implemented to obtain silicon micro-cantilevers patterned selectively with aluminum, as shown in Figure 5.3. The damping in the beams was measured by the standard procedures outlined in section 2.4 .

Subsequently, nine of the patterned beams were reused to systematically examine the effect of the length of the coating from the base of the cantilever on the damping in the composite. These nine beams were first wet etched in an aluminum etchant (Aluminun etchant type A, Transene Company Inc, USA) for 30 minutes, cleaned in de-ionized water and then wet etched in a chromium selective etchant (CR14F , Cyantec Corporation, USA) for 30 minutes. The damping in each of these cleaned beams was then re-measured and found to be within $5 \times 10^{-6}$ of the initial damping in the pristine beams. These beams were then divided into three sets (Set $\mathrm{E}-\mathrm{G})$ and re-patterned with $100 \mathrm{~nm}$ thick aluminum films following the method outlined above. The films were patterned to start at the base and extend to $0.4 L, 0.6 L$ and $0.8 L$ for the sets $\mathrm{E}, \mathrm{F}$ and $\mathrm{G}$ respectively. 
Table 5.1: Details of selective metallization for the four sets of beams measured in this study. Each set contained three beams (labeled A1, A2, A3, B1,..). The size and location of the aluminum patterns are specified by their start and end positions along the axis of the beam.

\begin{tabular}{llcc}
\hline & Beam Set & Start Position $\left(x_{1} / L\right)$ & End Position $\left(x_{2} / L\right)$ \\
\hline & Set A- Fully coated & 0 & 1 \\
\hline & Set B- Tip coated & $0.80-0.82$ & 1 \\
\hline & Set C- Base not coated & $0.17-0.20$ & 1 \\
\hline & Set D- Base coated 20\% & 0 & $0.15-0.17$ \\
\hline & Set F- 60\% Coated & 0 & $0.39-0.40$ \\
\hline
\end{tabular}

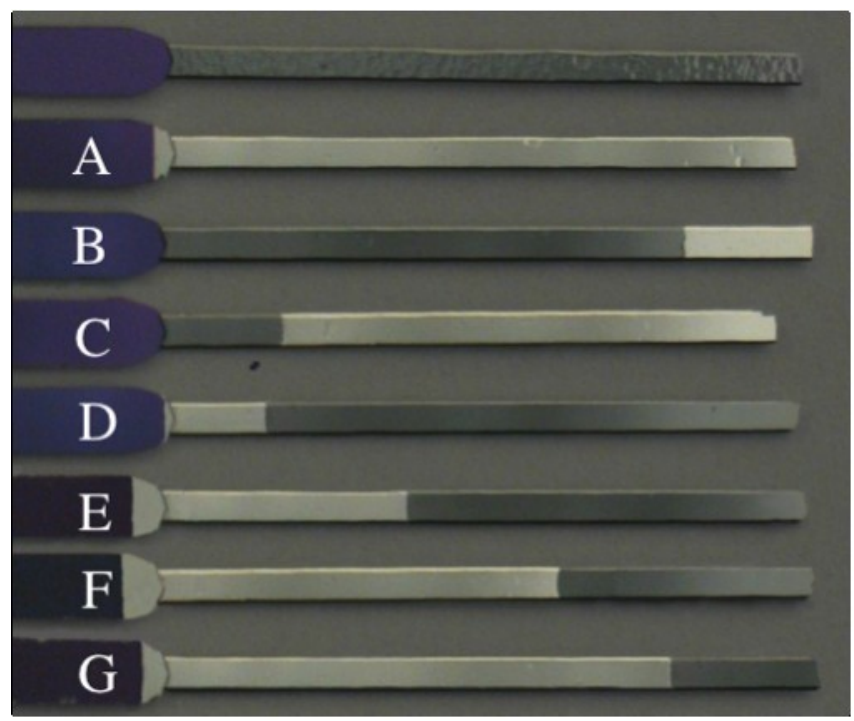

Figure 5.3: Photographs of representative single-crystal silicon beams patterned selectively with $100 \mathrm{~nm}$ thick films of aluminum. The labels correspond to the sets A-G shown in Table 5.1. Also shown is a representative bare silicon beam without any metallic coating. 


\subsection{Results and discussion}

Let us first examine the results of measurements on uncoated bare silicon beams. The measured natural frequencies of vibrations differed by less than $5 \%$ from the predictions of the standard Euler-Bernoulli model for cantilevers oscillating in the first mode. Across all twelve silicon beams, the logarithmic decrement was less than $1.6 \times 10^{-5}$ in the first mode. In all cases, thermoelastic damping is the dominant source of dissipation, and contributions from all other sources of dissipation (including anchor losses and clamping losses) are less than $10^{-5}$. The results of measurements on the full set of twelve beams are compiled in Tables 5.2. The tables list the dimensions of the microcantilevers, the frequency of oscillation, and the values of damping measured in the bare silicon and composite microcantilevers.

In addition to the measured values for dissipation, the table lists the predictions of Equation (5.6) for the damping in the partially-metalized silicon microcantilevers, $\delta_{c}^{E q(5.6)}$. These values were calculated by setting the internal friction in aluminum equal to $0.047 \pm 0.006( \pm 15 \%)$. This value was obtained from the study of the effects of frequency and thickness on internal friction in thin films of aluminum carried out in Chapter 3. The results in Table 5.2 show that all but one measurement is within the error in the predictions of Equation (5.6) using the previously measured value of internal friction. 
Table 5.2: Results of damping in the first mode of flexural vibration. The measured values of damping of the bare silicon $\left(\delta_{s}\right)$ and composite $\left(\delta_{c}\right)$ microcantilever are listed along with the values of damping in the composite predicted using Equation (5.6), $\delta_{c}^{E q(5.6)}$ * indicates beams that were reused in the study.

\begin{tabular}{|c|c|c|c|c|c|c|c|}
\hline $\begin{array}{c}\text { Beam } \\
\#\end{array}$ & $\begin{array}{c}h_{\mathrm{s}} \\
(\mu \mathrm{m})\end{array}$ & $\begin{array}{c}L_{s} \\
(\mathbf{m m})\end{array}$ & $f(\mathrm{~Hz})$ & $\delta_{s} \square 10^{-5}$ & $\delta_{c} \times 10^{-5}$ & $\delta_{c}^{E q(5.6)} \times 10^{-5}$ & $\frac{\delta_{c}-\delta_{c}^{E q(5.6)}}{\delta_{c}}$ \\
\hline A1 & 84 & 23.6 & 215.52 & 1.42 & 7.44 & 8.38 & $-11.2 \%$ \\
\hline A2 & 76 & 22.8 & 192.06 & 1.02 & 8.10 & 8.71 & $-6.9 \%$ \\
\hline A3 & 93 & 24.1 & 201.41 & 1.47 & 7.51 & 7.75 & $-3.0 \%$ \\
\hline B1 & 85 & 24.0 & 207.18 & 1.38 & 1.50 & 1.38 & $8.7 \%$ \\
\hline B2 & 85 & 23.2 & 223.20 & 1.53 & 1.47 & 1.53 & $-4.0 \%$ \\
\hline B3 & 73 & 23.1 & 188.13 & 1.13 & 1.08 & 1.14 & $-5.2 \%$ \\
\hline $\mathrm{C} 1$ & 79 & 22.6 & 222.63 & 1.37 & 3.83 & 4.87 & $21.4 \%$ \\
\hline $\mathrm{C} 2$ & 83 & 23.1 & 222.63 & 1.35 & 3.55 & 4.07 & $12.4 \%$ \\
\hline $\mathrm{C} 3$ & 86 & 23.8 & 220.05 & 1.54 & 4.08 & 4.53 & $9.9 \%$ \\
\hline D1 & 89 & 23.8 & 234.87 & 1.52 & 4.40 & 4.60 & $4.5 \%$ \\
\hline D2 & 79 & 23.0 & 204.27 & 1.13 & 4.89 & 4.85 & $-0.7 \%$ \\
\hline D3 & 79 & 23.0 & 213.29 & 1.11 & 4.53 & 4.49 & $-0.7 \%$ \\
\hline E1 & 89 & 23.8 & 234.87 & $* 1.57$ & 7.38 & 7.39 & $-0.1 \%$ \\
\hline E2 & 79 & 23.0 & 213.29 & $* 1.40$ & 7.94 & 7.98 & $-0.4 \%$ \\
\hline E3 & 73 & 23.1 & 188.13 & $* 0.98$ & 8.73 & 8.02 & $8.9 \%$ \\
\hline F1 & 79 & 23.0 & 204.27 & $* 1.39$ & 9.35 & 8.76 & $6.8 \%$ \\
\hline F2 & 86 & 23.8 & 220.05 & $* 1.48$ & 8.29 & 8.20 & $1.1 \%$ \\
\hline F3 & 93 & 24.1 & 201.41 & $* 1.66$ & 8.63 & 7.81 & $10.9 \%$ \\
\hline G1 & 85 & 24.0 & 207.18 & $* 1.40$ & 8.03 & 8.27 & $-2.8 \%$ \\
\hline G2 & 84 & 23.6 & 215.52 & $* 1.57$ & 9.47 & 9.25 & $2.4 \%$ \\
\hline
\end{tabular}


These results can be conveniently compiled using graphs that illustrate the relative increase in damping for different patterns of metallization as shown in Figure 5.4 and Figure 5.5. In these figures, measured damping due to metallization is normalized with respect to the predicted damping for a fully coated beam of the same thickness.

The curves in these figures show the predicted increase in damping in the composite due to the addition of the film for the first mode of vibration. The predicted values were computed by piecewise integration of the curves shown in Figure 5.2. The curve in Figure 5.5 differs from that in Figure 5.4 only in the manner in which the limits of integration are imposed; that is, either from the root $(0 \rightarrow \xi)$ or from the tip of the beam $(\xi \rightarrow 1)$. The use of each curve depends solely on the geometry of the metalized patch.

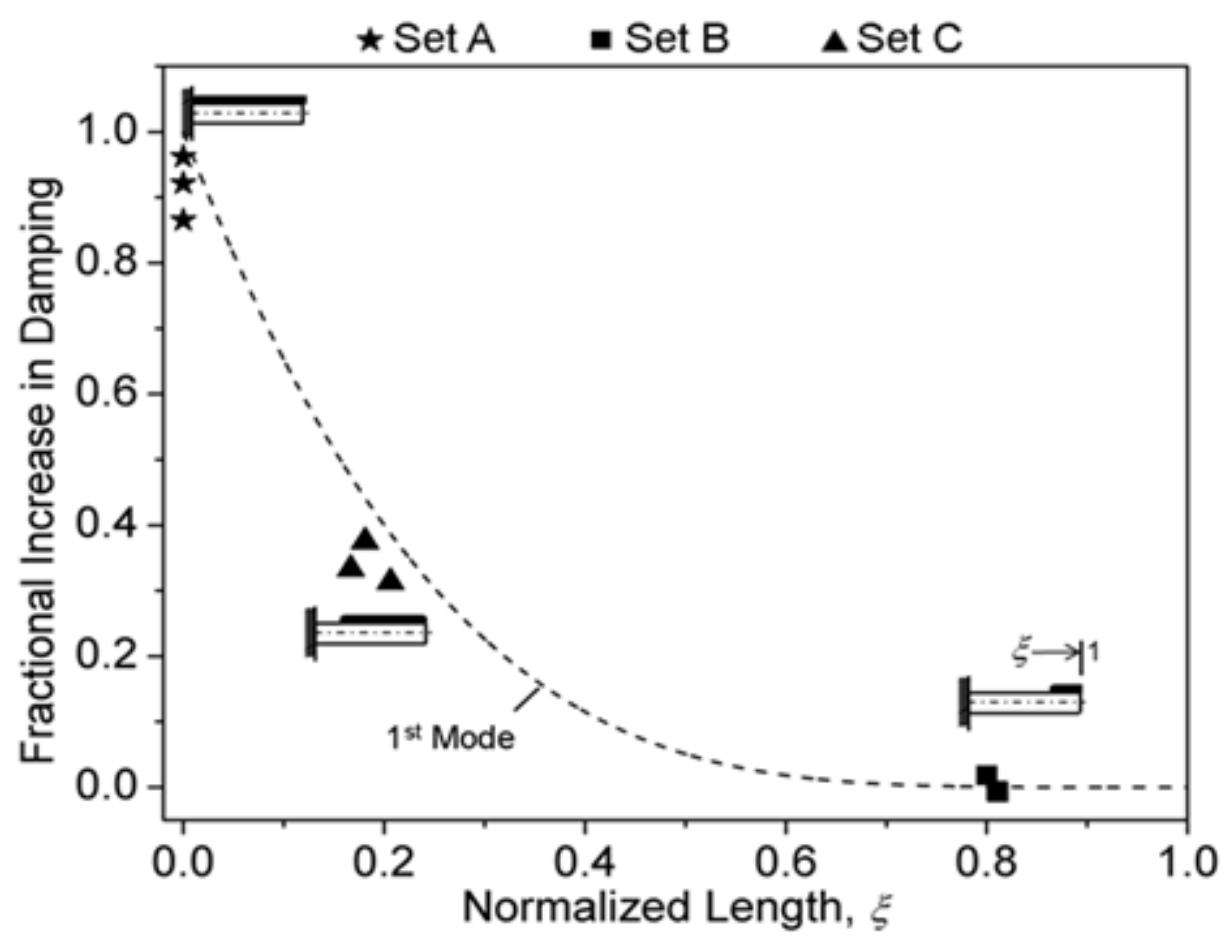

Figure 5.4: Graph of the measured increase in damping due to metallization as a function of pattern geometry for microcantilevers vibrating in the first mode. These values are normalized with respect to the predicted damping for a fully coated beam of the same thickness. The curve represents the predicted fractional increase in dissipation with patterning along the length of the cantilever starting from the tip and was calculated from Equation (5.6). 
From Figure 5.4 we see that a metallic patch as long as $20 \%$ of the length of the beam can be patterned at the tip of the cantilever without incurring any significant increase in damping. In contrast, Figure 5.5 shows that a patch of the same length, but located at the root, leads to a large increase in damping. Indeed, the increase due to this patch is $\sim 60 \%$ of that due to a full coat. Taken together, the measurements shown in Figure 5.4 and Figure 5.5 match well with the predicted values of damping and validate the use of the simple model outlined in section 5.1. The results confirm the effectiveness of selective metallization as a strategy for controlling damping and quality factors.

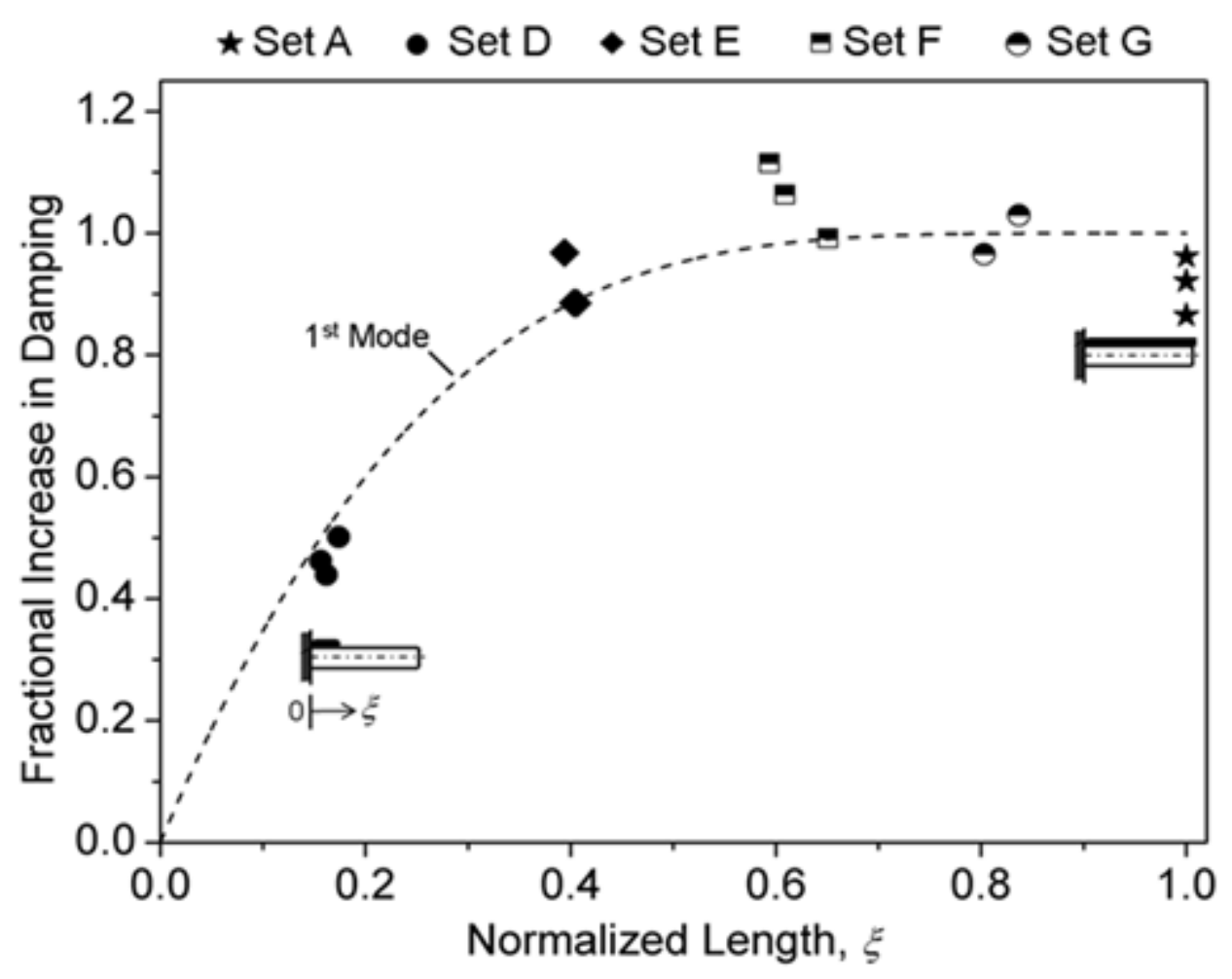

Figure 5.5: Graph of the measured increase in damping due to metallization as a function of pattern geometry for microcantilevers vibrating in the first mode. These values are normalized with respect to the predicted damping for a fully coated beam of the same thickness. The curve represents the predicted fractional increase in dissipation with patterning along the length of the cantilever starting from the base and was calculated from Equation (5.6). 
The insight with this approach can have an immediate impact on the design of high-Q/low damping resonators. Consider for instance, applications such as atomic force microscopy where the location of the tip is monitored using optical techniques. A reflective metallic patch covering up to $0.4 \mathrm{~L}$ of the tip can provide sufficient optical reflectivity without degrading the $Q$ factor. Indeed, this principle can be applied to displacement based flexural resonators, such as those operated by electrostatic actuation. Conversely, in applications where low damping is sought after but high strain energies are required, (such as in piezoelectric vibration energy harvesting), suitable tradeoffs between damping and strain can be achieved by controlling the dimensions of the films at the base of the cantilever.

\subsection{Summary}

This chapter presented an approach for preserving functionality and controlling dissipation by selective patterning of thin film materials in regions of low deformation. A simple model based on the distribution of strain energy along the length of the cantilever was developed to predict the increase in damping with partial metallization. The validity of the model was then experimentally tested using single-crystal silicon microcantilevers operating near their fundamental thermoelastic damping limit that were selectively coated with Al to isolate the impact of metallization on dissipation. The measured increase due to metallization was found to be in good agreement with the predictions. The quantitative guidelines that emerge from this work can be used for reducing dissipation in devices such as commercial silicon microcantilevers used for atomic force microscopy. 


\section{Chapter 6}

\section{The Design of a Microcantilever System to Measure Damping as a Function of Temperature.}

\section{Overview}

The first five chapters of this dissertation all discuss and present measurements of internal friction at room temperature. This chapter describes the first steps to the development of a measurement system that is capable of measuring damping in thin films as a function of temperature $\left(20^{\circ} \mathrm{C}-300^{\circ} \mathrm{C}\right)$. Such measurements are a powerful approach to uncover the underlying mechanisms responsible for the high dissipation we have seen in thin films.

Previous studies of internal friction in thin film materials such as Al [Ber75, Pri94, Cho04)], Ag [Kin94], $\mathrm{Au}$ [Nis06], and $\mathrm{Cu}$ [Cho06] have shown that a component of the internal friction observed in these films is anelastic in nature. The first section of this chapter provides a brief description of dissipation due to anelasticity and then shows the need for studies of dissipation over both frequency and temperature to determine the nature of the damping mechanisms. Subsequently, the design requirements and the development of a high temperature damping measurement system are described. Finally, the first measurements of damping using the setup are presented and described.

\subsection{Anelastic damping in thin films}

The discussion of anelasticity follows the work of Nowick and Berry [Now72]. Anelasticity involves a recoverable time dependent strain in response to an applied stress. Figure 6.1 (a) describes the behavior of such a material to a constant stress. When subject to a uniaxial stress $\sigma_{0}$, the deformation consists of an instantaneous un-relaxed (or elastic) response $\varepsilon_{U}$, followed by a strain 


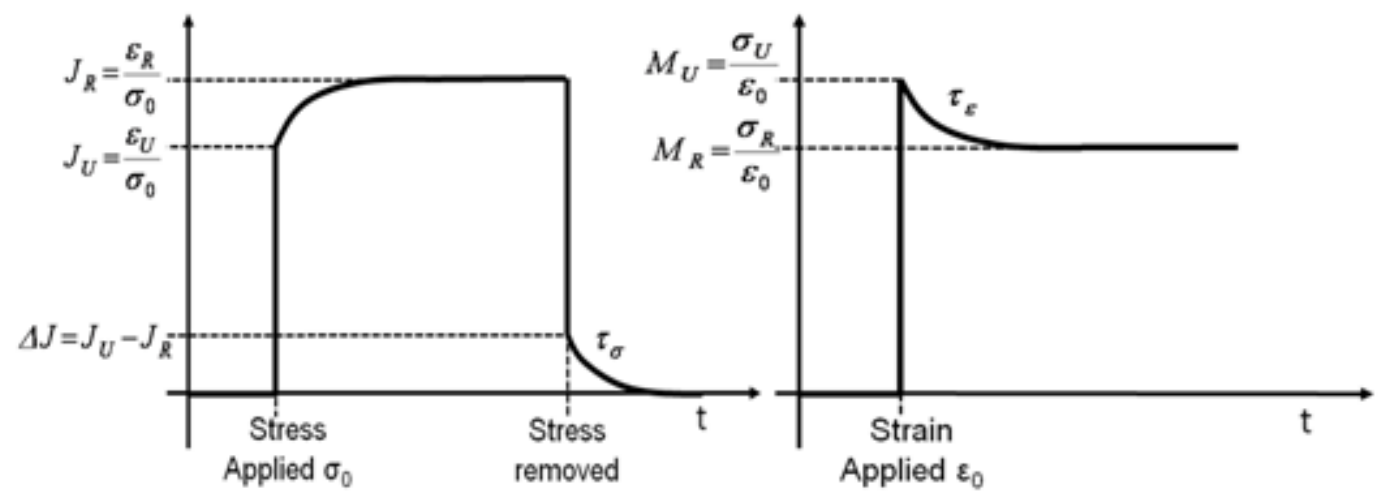

Figure 6.1: Schematic illustration of anelastic behavior with time: a) Strain response to an instantaneous applied stress. $J_{R}$ and $J_{U}$, are the relaxed and unrelaxed compliance respectively. $\Delta J$ is the relaxation strength and $\tau_{\sigma}$ is the relaxation time at constant stress. b) Stress response to an instantaneous application of strain. $M_{R}, M_{U}$ and $\tau_{\varepsilon}$ are the relaxed, un-relaxed and relaxation time constant at constant strain, respectively [Meh07].

relaxation to obtain a final relaxed strain $\varepsilon_{R}$. This relaxation, commonly referred to as creep [Now72], occurs over a period of time known as the relaxation time at constant stress $\tau_{i}$. Upon unloading, the material returns to its original unstrained form, however, also with the same time period for recovery. Conversely, when subject to a constant strain (Figure 6.2b) the material experiences an instantaneous stress which then relaxes over a period of time, called the relaxation time at constant strain, $\tau_{2}$.

The behavior of this material is captured by a three parameter, "standard anelastic solid" model that was developed by Zener [Now72]. Figure 6.2 shows the compliance version of the model wherein $J_{U}$ is the un-relaxed compliance, $J_{R}$ is the relaxed compliance, $\Delta J=J_{U}-J_{R}$ is the difference between the relaxed and un-relaxed compliance, and $\tau_{\sigma}$ is the relaxation time at constant stress. 


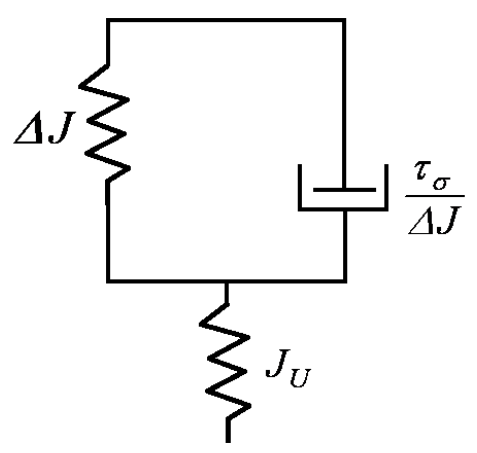

Figure 6.2: Standard anelastic solid model where $J_{R}$ and $J_{U}$, are the relaxed and unrelaxed compliance respectively. $\Delta J$ is the relaxation strength and $\tau_{\sigma}$ is the relaxation time at constant stress [Now72].

In this work, we are interested in the dynamic response of this material and in particular the damping that results from the lag between stress and strain. For the standard anelastic solid, the damping in the material as a function of frequency, $\omega$, is given by [Now72]

$$
\delta=\pi \frac{\Delta J}{\left(J_{U} J_{R}\right)^{1 / 2}} \frac{\omega \tau}{\left(1+\omega^{2} \tau^{2}\right)}
$$

where $\tau$ is the mean relaxation time and is given by[Now72]

$$
\tau=\sqrt{\tau_{\sigma} \tau_{\varepsilon}}
$$

Equation (6.1) is a characteristic anelastic damping curve that has a peak at $\omega \tau=1$, which implies that a peak in damping is produced when the frequency of oscillation matches the relaxation time of the underlying process. Many anelastic processes have relaxation processes that are thermally activated and follow an Arrhenius equation of the form [Meh07],

$$
\tau=\tau_{0} \exp \left(H / k_{B} T\right)
$$

where $\tau_{0}$ is the pre-exponential diffusion factor, $H$ is the activation energy, $k_{B}$ is the Boltzmann's constant and $T$ is the temperature of the process. Therefore by varying the temperature one can alter the characteristic relaxation time of the 
process. By substituting Equation (6.3) into Equation (6.1), we can see that the same damping behavior can be observed by either varying the frequency at a constant temperature or by varying the temperature at a constant frequency. In both cases, the resulting damping traces a characteristic anelastic frequency response peak similar to that for TED (Figure 2.2). By measuring the damping over a range of both frequency and temperature, one can determine two vital pieces of information: (i) the presence of an anelastic damping mechanism and (ii) an estimation of the activation energies associated with the mechanism. This motivated the development of a damping measurement platform to measure the internal friction in thin film as a function of both frequency and temperature.

\subsection{Design of high temperature setup:}

The three main design requirements for a high temperature measurement setup were as follows:

i) The system should be capable of measuring damping over a temperature range of $295 \mathrm{~K}$ to $\sim 700 \mathrm{~K}$ and a frequency range of $100 \mathrm{~Hz}$ to $100 \mathrm{kHz}$.

ii) The system should utilize the single-crystal silicon beams developed in Chapter 2 and 4.

iii) The guidelines developed to minimize viscous, clamping and support losses should be implemented such that beams should approach the limits imposed by TED with the same, or less, clamping loss.

Adding a temperature control circuit to the measurement setup described in Chapter 2 was not possible as the shaker assembly was based on piezoelectric actuation. At high temperatures, a piezoelectric material depolarizes and does not exhibit bulk directional poling upon cooling. To overcome this drawback, a new actuation method had to be selected and therefore required the design of an entirely separate system for high temperature measurements.

The detailed design and development of an electrostatic actuation based setup was developed by Nabil Shalabi [Sha11] as part of his Master's thesis. What follows is 
a brief overview of the system. Figure 6.3 shows a schematic of the high temperature damping measurement setup. The base of the setup consists of a precision machined stainless steel block, mounted onto a Macor (Dow Corning) insulating ceramic block. Mounted onto the base is a simplified and enhanced clamp mechanism.

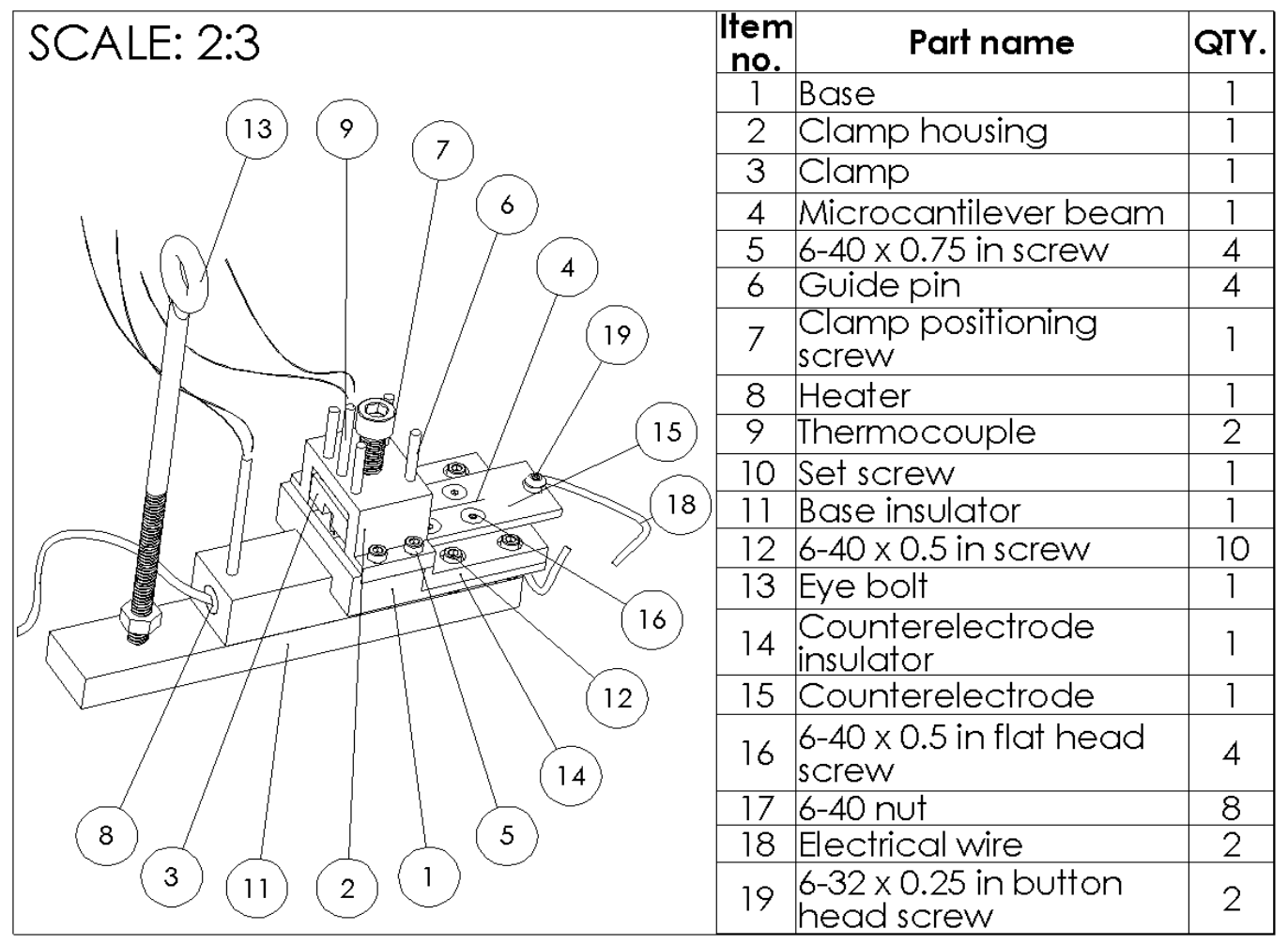

Figure 6.3: Design schematic of high temperature damping measurement setup using electrostatic actuation [Image from Sha11].

The setup is designed to accept beams of the type described in Chapter 2 and Chapter 4. The clamp is designed much like a vertical press with a large single screw acting as the drive and applying pressure directly on the clamped beam. This design eliminated the need for multiple screws and ensured that the clamping force was centered. The clamping surfaces were, once again, precision machined for parallelism and flatness following the guidelines in Chapter 2. 
The beams are actuated electrostatically and are therefore required to be conductive. The conventional technique of applying a thin conductive film to the resonator was not an option as it is the damping in these very films that we are trying to study. A simple and suitable alternative was the use of low resistivity (Ptype doped) silicon wafers as the starting material.

Electrostatic actuation of the beam is achieved by first clamping the beam firmly in place. Once loaded, the base of the beam is in electrical contact with the clamp (3) and forms the primary electrode while the free end is suspended over a secondary electrode (15). In effect, the beam and second electrode can be imagined to be two parallel plates. Applying a potential difference between the suspended beam and the underlying electrode, forces the beam to bend. Removal of the potential difference forces the beam to spring back to its unstrained state. Therefore, the beam can be oscillated by applying a cyclic potential difference between the beam and the bottom electrode.

Heating of the specimen is achieved by a cartridge heater that is mounted into the base of the setup. The temperature is measured with the help of two thermocouples: one located on the clamp and the other on the heater. The thermocouples used in this design are R-type thermocouples (rhodium and platinum leads) which are brought into point contact with the heated surfaces. The output of the thermocouples is fed into a programmable temperature controller which allows for precise control of the temperature on the clamp. The thermocouples are fed into the chamber by a commercially purchased R-Type thermocouple feedthrough (MDC Vacuum Incorporated, USA). Finally, the entire setup can be lowered into the vacuum chamber and measurements of damping in the beam can be carried out as outlined in chapter 2 . 


\subsection{Initial validation}

\subsubsection{Clamping loss}

Initial measurements were carried out with beams fabricated from highly doped, low resistivity silicon wafers $(0.001 \Omega / \mathrm{cm})$. The beams were first fabricated by the process outlined in Chapter 2. This process retains a $500 \mathrm{~nm}$ layer of $\mathrm{SiO}_{2}$ on the base and acts as an insulator. Therefore, to establish an electrical contact with the silicon beam, one side of this insulating oxide was then etched away by reactive ion etching. A small section of the base was sputter coated with $30 \mathrm{~nm}$ of gold and a $10 \mathrm{~nm}$ titanium adhesion layer in order to achieve good electrical contact.

The damping in the beams was first measured with the piezoelectric shaker system to determine the resonant frequency and compare the clamping losses between the two setups. The beams were then loaded into the electrostatic shaker setup and clamped in place by screwing down the clamp. The clamp screw was systematically tightened to a torque value of $1.5 \mathrm{Nm}$. The beam was then excited with a time harmonic voltage signal from the function generator. No DC offset was utilized and therefore the excitation frequency is half that of the resonant frequency of the cantilever. To excite the tip of the beam to a velocity of $5 \mathrm{mms}^{-1}$ required $4 \mathrm{~V}$. The damping in the beam was extracted by the procedure described in Chapter 2. Measurements of three beams with both the electrostatic and piezoelectric shaker assembly were similar indicating that both systems had similar clamping losses (Table 6.1). 
Table 6.1: Results of damping measurements with the piezoelectric shaker and electrostatic device described in section 6.2. All measurements were under high vacuum and room temperature.

\begin{tabular}{ccccccc}
\hline $\begin{array}{c}h_{s} \\
(\mu \mathrm{m})\end{array}$ & $\begin{array}{c}\text { Length } \\
(\mathrm{mm})\end{array}$ & $\begin{array}{c}\text { Measured } 1^{\text {st }} \\
\text { mode } \\
\text { frequency } \\
(\mathrm{Hz})\end{array}$ & $\begin{array}{c}\delta_{s, T E D} \\
\times 10^{-5}\end{array}$ & $\begin{array}{c}\delta_{s} \times 10^{-5} \\
\text { Shaker }\end{array}$ & $\begin{array}{c}\delta_{s} \times 10^{-5} \\
\text { Electro } \\
\text { Static }\end{array}$ & $\begin{array}{c}\delta_{s}-\delta_{s, T E D} \\
\times 10^{-5}\end{array}$ \\
\hline 96 & 33 & 122.9 & 0.31 & 1.38 & 1.51 & 0.12 \\
120 & 27 & 208.4 & 1.38 & 2.71 & 2.78 & 0.07 \\
118 & 18.3 & 456.1 & 2.92 & 4.82 & 4.83 & 0.007 \\
\hline
\end{tabular}

\section{Temperature control capability}

Initial tests of the temperature control system were carried out at atmospheric pressure. The setup, with beam mounted, was heated up to $350^{\circ} \mathrm{C}$ in ramp steps of $50^{\circ} \mathrm{C}$. The setup was held at each temperature for 8 minutes. Figure 6.5 demonstrates the performance of the temperature controller in air.

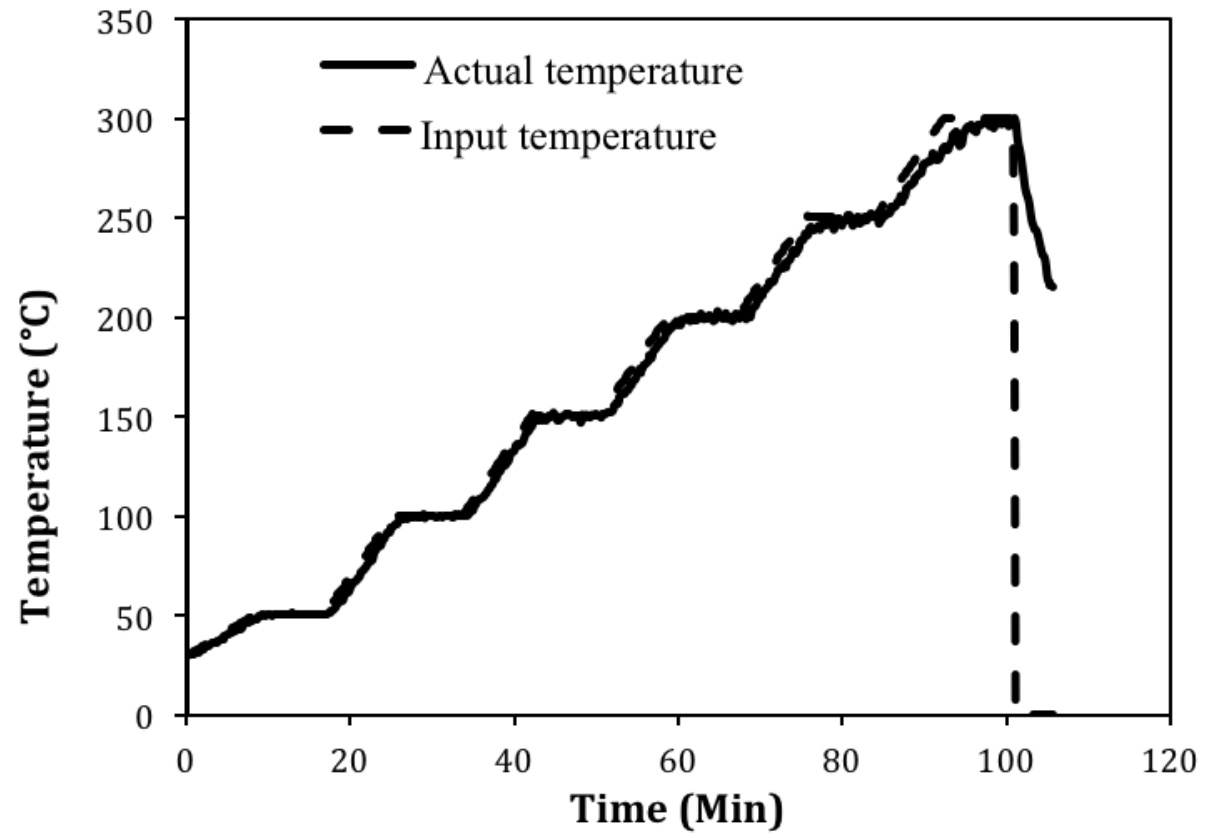

Figure 6.4: Plot of temperature as a function of time comparing the actual temperature of the clamp to the input set-point temperature. These measurements were carried out under ambient pressure conditions. Graph reproduced from [Sha11]. 
Unfortunately, measurements under vacuum were not possible due to the inability of the thermocouples to accurately measure the temperature on the clamp or the heater under vacuum conditions.

\subsection{Required modifications}

As with all new designs, this setup requires several design iterations to improve its performance. There are two main issues that need to be addressed:

i. The poor performance of the thermocouples under vacuum is the single hurdle preventing measurements of damping as a function of temperature. The issue lies in the contact of the thermocouple with the heater and clamp surface. There are two possible issues here: The first is that the present design relies on the weight of the thermocouple to contact with the clamp. Applying a small pressure to the junction of the thermocouple may improve its performance. Alternately, the single point contact of the junction is a possible source for high thermal contact resistances. A redesign of the thermocouples to be permanently integrated into the body of the clamp assembly may rectify this problem.

ii. The drive amplitude for lightly damped low frequency beams was $\pm 4 \mathrm{~V}$. It is reasonable to assume that the driving voltages at resonance for a more heavily damped beam would be significantly higher. For highly damped beams the required drive voltages may exceed the current capabilities of the function generator and must therefore be systematically studied. This issue can be overcome by using a function generator with a higher voltage capability. Alternately, the driving voltage can be reduced by decreasing the gap between the beam and lower electrode. The beams utilized in this thesis have gap sizes ranging from $250-600 \mu \mathrm{m}$. By altering the process flow and utilizing thinner wafers, this gap size can be reduced significantly. 


\subsection{Summary}

This chapter described the development of a high temperature system utilizing electrostatic actuation and optical detection to measure damping in thin films. The system was tested for actuation, temperature control and measurements of damping. Through careful design of the clamping setup, the system was demonstrated to operate doped single-crystal silicon beams with low clamping and support losses at room temperature. These are the first steps to the development of a high temperature damping measurement that utilizes TED as the basis for calibration. 


\section{Conclusions and Future work}

\section{Conclusion}

Measurements of damping in thin films can provide useful guidelines for the design of layered, composite micro and nanoscale resonators widely used for sensing, signal filtering and energy harvesting. This thesis developed a novel approach for the measurement of internal friction in thin films using thermoelastic damping (TED) as the basis for calibration. The procedure can be summarized in four steps: first, single-crystal silicon cantilevers are demonstrated to operate close to the TED limit showing that viscous, clamping and support losses have been adequately minimized. The beams are then coated with the thin film of interest taking care not to change the clamping conditions. The damping in the layered composite is then measured. For a thin film on a thick substrate, there is a negligible change in the TED as a result of layering. Therefore, we can subtract the initial damping in the substrate from the measured damping in the composite to obtain an accurate measure of the damping in the film.

The detailed design and development of a damping measurement platform was described. Through careful analysis of clamping, support and viscous losses, the system was shown to be capable of operating single-crystal microresonators to

within $1 \times 10^{-5}$ of the TED limit in a frequency range from $70 \mathrm{~Hz}$ to $40 \mathrm{kHz}$. The system was then applied to three main studies of the measurement and impact of damping in thin metallic films. The results of the three studies can be compiled to provide some valuable guidelines for the design of layered resonators and some insight into the internal friction mechanisms in thin sputter deposited aluminum films. 


\subsection{Guidelines for the design of layered resonators}

Thin polycrystalline metallic films used as functional layers in resonators can lead to a disproportionate increase in the dissipation in the structure. To minimize the dissipative impact while maintaining functionality, there are three main strategies that can be employed:

1) Minimize the amount of dissipative thin film material used in the resonator

2) Use films with lower internal friction

Empirical data on the damping in thin film materials would enable the selection of low dissipation materials while preserving functionality. This thesis demonstrates that low stress aluminum films used as reflective coatings dissipate more than gold or silver over a frequency range from 100-1500 Hz. In addition, $\mathrm{Al}$ films have high values of dissipation (0.03) up to frequencies of $40 \mathrm{kHz}$.

3) Where possible, pattern films in regions of low strain on the resonator The dissipation in the films is proportional to the amount of deformation it experiences. By carefully considering the vibration mode shapes of the resonator one can determine suitable locations to place thin film layers such that the functionality is retained but the dissipation is minimized. 


\subsection{Studies on internal friction in thin films as a function of frequency}

In comparison to internal friction in bulk solids, the mechanisms of internal friction in thin films have not been widely studied. In addition, most studies have been carried out as a function of temperature to identify the dominant mechanisms. With the implications of dissipation on the performance of composite micro and nano-resonators, an understanding of the underlying mechanisms of thin film damping as a function of frequency is becoming increasingly important.

In this thesis, the first steps towards understanding dissipation in thin films of aluminum as a function of frequency were demonstrated. $60 \mathrm{~nm}$ thick aluminum films showed a twofold decrease in internal friction over three decades of frequency from $70 \mathrm{~Hz}$ to $40 \mathrm{kHz}$. If the dissipation were due to a single mechanism, the damping is expected to have decreased by an order of magnitude over the same range. It was verified that an increase in TED in the composite did not account for the measured change. The identities of the mechanisms of internal friction that are responsible for this frequency dependence is a topic of future research. The difficulty of this task can be placed in perspective by noting that even after decades of research, there are no reliable models to predict the frequency dependence of internal friction in bulk polycrystalline materials (such as $\mathrm{Al}, \mathrm{Au}, \mathrm{Ag})$. 


\subsection{Future work}

There are a multitude of interesting and important studies that can be carried out with this platform as it exists. Studies on the effect of annealing in thin films and measurement of damping in nanowires of aluminum have already been conducted with interesting results. Several other interesting investigations are listed below:

\section{1) The effect of mode shape on damping in thin films}

The single-crystal silicon beams used in this thesis are lightly damped and the modes are well spaced in frequency. Of interest here would be to examine the effect of mode shapes on damping in the thin films. An approach to carrying out these measurements and preliminary results for measurements of silicon beams are shown in the appendix A1.

2) Accurate measurements of internal friction in the $100 \mathrm{kHz}$ to $100 \mathrm{MHz}$ range.

At present, measurements of internal friction at these frequencies have been limited to wave propagation techniques to study dissipation in bulk specimens only. No system that can achieve these measurements has been demonstrated to date. Using the procedure outlined in chapter 4 , the platform can be extended to make the first accurate measurements of internal friction in this frequency range. Such measurements would apply to a much wider spectrum of microscale resonators in operation at present.

To do this would require an enhancement of the data acquisition system, a modification of the decoder in the LDV and the appropriate shielding for high frequency electromagnetic interference.

\section{3) Smallest measurements of damping}

Two factors that currently limit the smallest damping that can be measured are the clamping loss and the low frequency excitation affecting the laser signal. It is not clear what factors affect the clamping loss in the current design. This loss does not correlate with beam geometry, frequency of operation, the type of starting 
substrate (single wafer or SOI) or the processing of the beams (TMAH or RIE) a One variable that was not considered was to vary the lateral dimensions of the base of the cantilever. Barring this modification, alternate designs of the resonator should be considered. One possible candidate is the free-free beam design with anchors located at the nodal points. Modifications to the current mount and setup would have to be considered to clamp the surrounding structure of the beam but are not too difficult to accomplish. However, one drawback of this approach is that these beams can only operate at one frequency.

To tackle the low frequency vibrations of the table, isolation of the pumping system and appropriately increasing the mass on the table can significantly reduce the signature.

\section{4) Wafer scale measurement of damping in thin films}

The procedure outlined in Chapter 4 provides the opportunity for the development of a wafer scale damping measurement setup. Such a setup would provide two exciting opportunities:

- At present, a microfabrication facility with a thin film deposition process cannot provide a designer with specifications regarding the dissipation properties of the film. The development of a wafer scale measurement tool would allow seamless integration into their existing processes in much the same manner film stress measurement tools operate today.

- Measurements of internal friction in thin films have always required a custom designed setup with custom designed measurement reeds. There is no doubt that this would have hindered studies to better understand the sources of dissipation in thin films. The development of a wafer scale tool has the potential to open the field to provide wider and easier access to this field of research.

The work in Chapter 4 provides the foundation for the development of such a tool. The cantilevers used for the study in Chapter 4 were all produced on a single wafer. With careful design a single SOI wafer can be used to produce cantilevers 
with frequencies over several decades ranging into the $10^{7} \mathrm{~Hz}$. Such a wafer could be introduced into a variety of deposition machines (Sputter, evaporation, molecular beam epitaxy). The actuation of the cantilevers can be easily achieved by electrostatic means. However, the development that is required here is in a clamping system and an approach to measure the vibration of a multitude of beams. 


\section{Appendix}

\section{A. Measurements of damping in higher resonant modes}

Preliminary measurements of damping in higher modes were accomplished by exciting the beam close to the frequency of the desired resonant mode. Once again, the excitation was terminated and the free decay recorded. The decay signal typically consisted of a superposition of several modes of oscillation as shown in Figure A1.1.
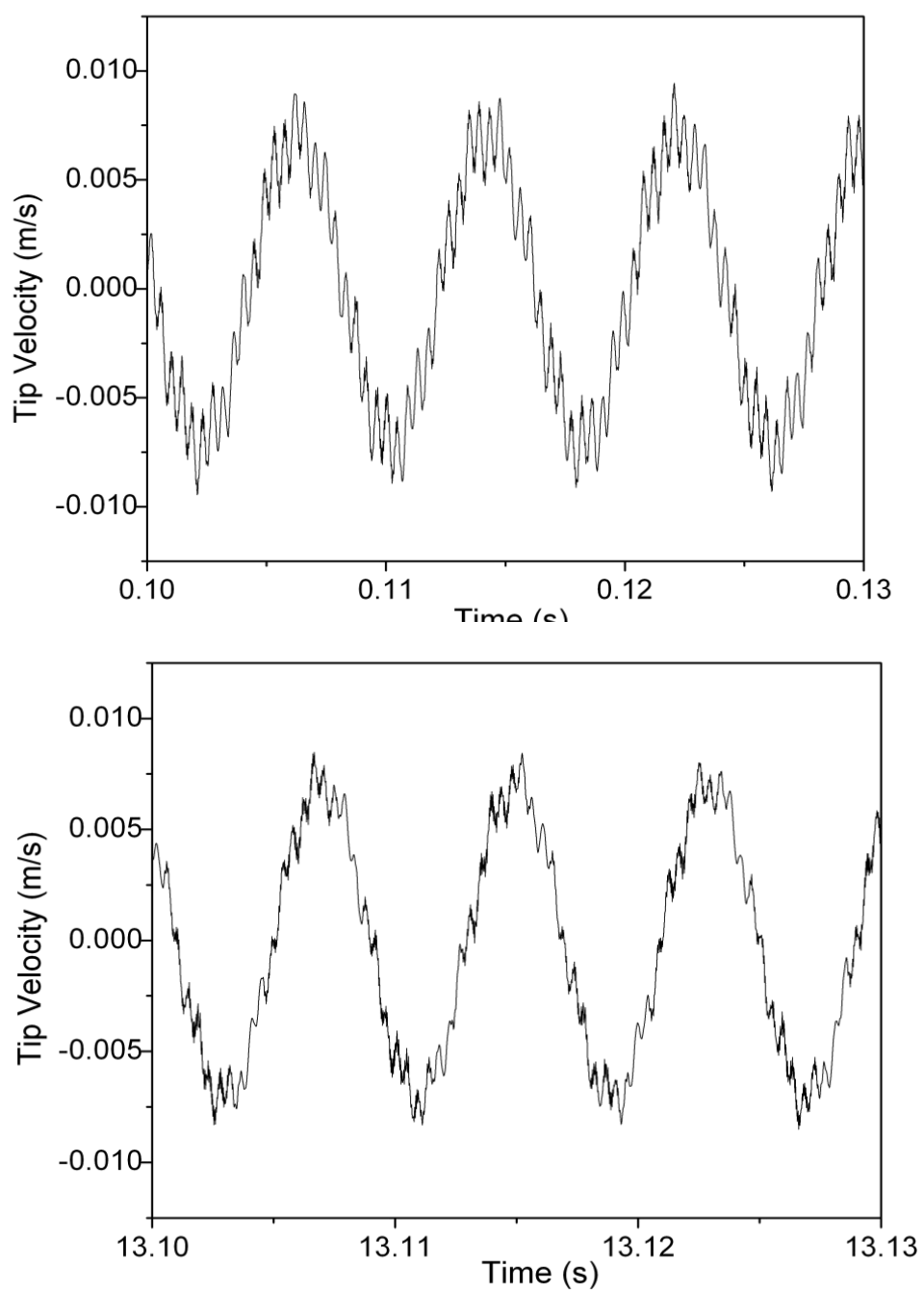

Figure A1.1: Decay of lightly damped beam excited in the first mode then in the third mode. a) Immediately after cutting the excitation b) after 13 seconds showing weaker $3^{\text {rd }}$ mode signal. 
To extract the damping in each mode, the measured free decay time response signal is first transformed to the frequency domain by a digital fast Fourier transformation (DFFT). Typically, the damping in the beams is low and the modal frequencies are well separated. Therefore, a simple peak-picking algorithm is applied to the power spectrum to identify the modal frequencies [Ben93]. The measured modal frequencies are compared and verified against the modal frequencies predicted by the standard model for a clamped-free Euler-Bernoulli beam. Each mode is then sequentially isolated by filtering the time domain signal with a three pole Butterworth band pass filter [Kim05, Bri00]. The bandwidth of the filter is selected by visual inspection, or by where the magnitude of the spectral response falls to the noise floor on either side of each resonance peak. The filtered response is effectively that of a system with a single degree-offreedom. The logarithmic decrement is then obtained from the slope of the logarithm of the envelope of the free decay using the Hilbert transform. To verify this approach, the decay values for filtered and unfiltered signals for the first mode were compared and showed no difference in measured damping. Figure A1.2 illustrates this procedure on the decay of a lightly damped silicon beam excited close to the third mode. From Figure A1.2(a) we see the distinct and well separated decay signatures of the three modes. The original time domain signal was then filtered to isolate the decay of each mode. Figure A1.2(b) shows the decay envelope of each of the modes using the Hilbert transform. 
(a)
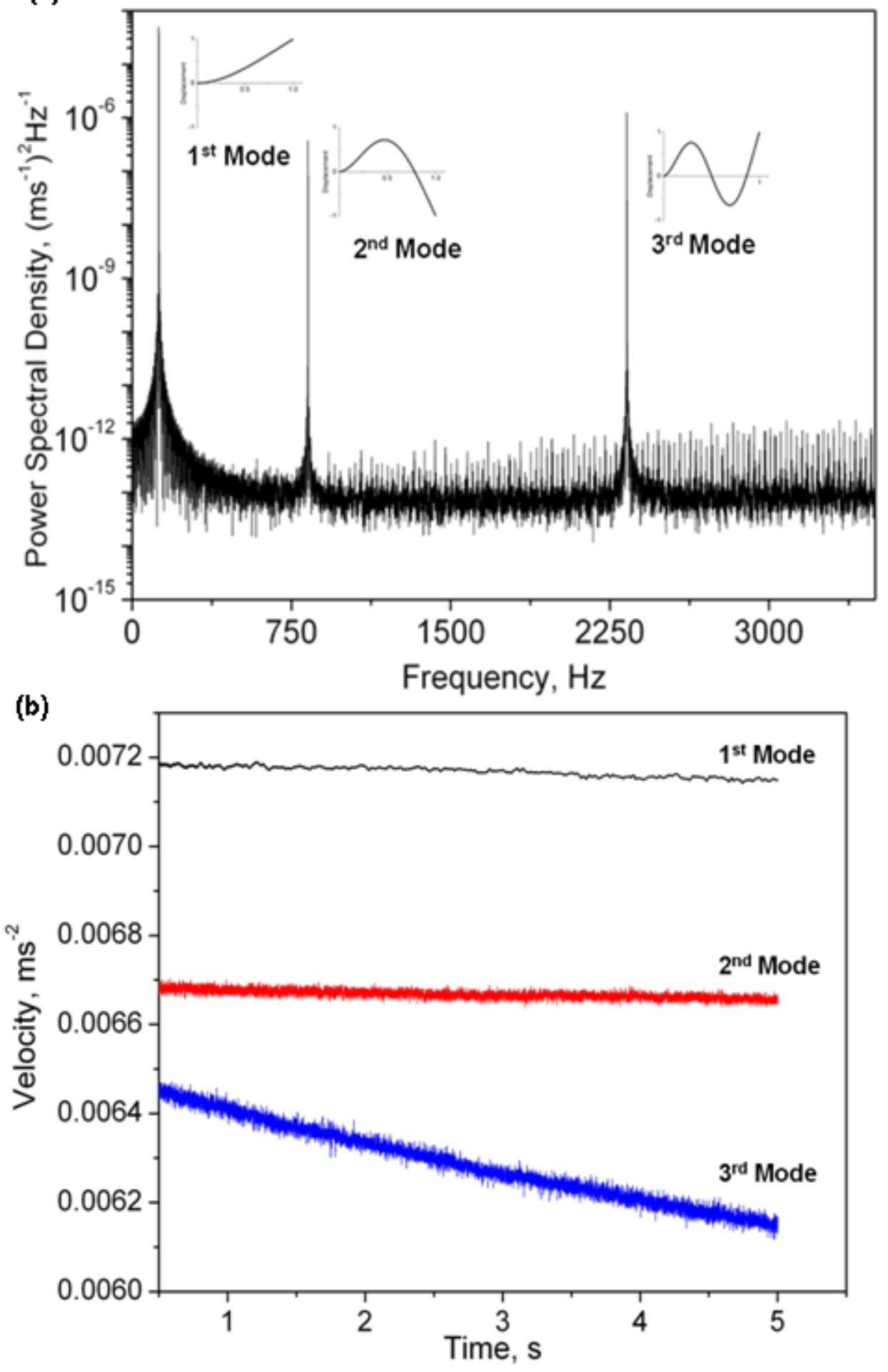

Figure A1.2: a) Power spectral density of silicon cantilever decaying from excitation close to the $3^{\text {rd }}$ resonant mode. The modes are lightly damped and are isolated by band pass filtering, $b$ ) the envelope of the decay signals for each isolated mode from calculated using equation 2.31. The values of the $2^{\text {nd }}$ and $3^{\text {rd }}$ mode have been offset to fit on the same scale. 
Preliminary measurements of damping in higher modes were carried out for a subset of single-crystal silicon beams used in Chapter 2. Figure A1.3 shows a graph of the logarithmic decrement versus frequency for a single-crystal silicon beam operating in the first four modes.

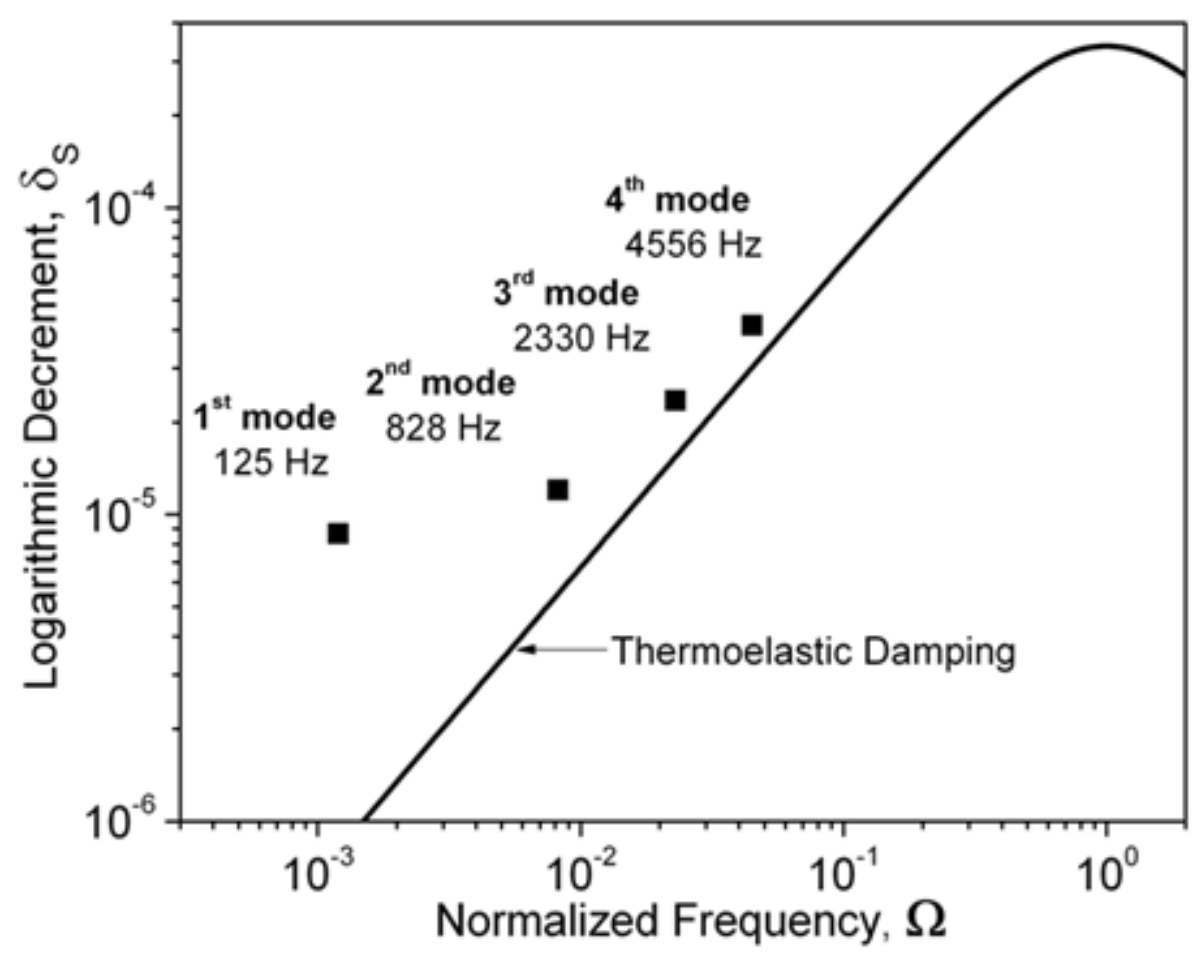

Figure A1.3: Logarithmic decrement as a function of normalized frequency for a $40 \mu \mathrm{m}$ thick cantilever measured in the first four resonant modes.

The curve shown in Figure A1.3 is once again calculated from Zener's expression and a recent two-dimensional model of thermoelastic damping confirms that this expression is accurate to within a few percent for the first four modes of these cantilever beams [Pra08]. The damping in the beams follows the thermoelastic damping curve with a no little change in the clamping loss. The measured decay frequencies differ once again, by less than $7 \%$ from the predicted modal frequencies of an Euler Bernoulli beam as shown in Table A1.1. 
Table A1.1: Comparison of measured and predicted decay frequencies for each mode of the lightly damped silicon beam shown in Figure A1.3. The beam is 20.8 $\mathrm{mm}$ in length and $40 \mu \mathrm{m}$ in thickness.

\begin{tabular}{ccccc}
\hline & Mode shape & Predicted frequency & Measured & \% difference \\
& factor $\left(\alpha_{n}\right)$ & $\frac{\alpha_{n}^{2}}{2 \pi L} \sqrt{\frac{E I}{\rho A}}$ & $\begin{array}{c}\text { Frequency } \\
(\mathbf{H z})\end{array}$ & \\
\hline $1^{\text {st }}$ Mode & 1.875 & 124 & 125.6 & 1.2 \\
$2^{\text {nd }}$ Mode & 4.694 & 777 & 828 & 6.5 \\
$3^{\text {rd }}$ Mode & 7.854 & 2176 & 2330 & 7.0 \\
$4^{\text {th }}$ Mode & 10.995 & 4264 & 4556 & 6.8 \\
\hline
\end{tabular}




\section{B. Background Data}

This section contains detailed information for the graphs shown in sections 2 and 3. Table B. 1 shows the data for the pristine single crystal silicon beams shown in Figure 2.2 while Table B.2 presents the data for the measurements in Figure 3.1113.

Table B.1: Measurements of pristine single crystal silicon beams shown in Figure 2.2

\begin{tabular}{|c|c|c|c|c|c|c|c|c|}
\hline & $\begin{array}{c}\text { Thickness } \\
(\mu \mathrm{m})\end{array}$ & $\begin{array}{c}\text { Length } \\
\text { (m) }\end{array}$ & $\mathrm{L} / \mathrm{H}$ & $\begin{array}{l}\text { Frequency } \\
\text { (Hz) }\end{array}$ & Omega & $\begin{array}{l}\delta_{S} \\
\times 10^{-5}\end{array}$ & $\begin{array}{l}\delta_{S, T E D} \\
\times 10^{-5}\end{array}$ & $\begin{array}{c}\left(\delta_{S}-\delta_{S, T E D}\right) \\
\square 10^{-6}\end{array}$ \\
\hline 1 & 117 & 0.036 & 308 & 114.375 & 0.01070 & 2.11 & 0.72 & 13.9 \\
\hline 2 & 117 & 0.02532 & 216 & 220.68 & 0.02065 & 2.05 & 1.39 & 6.61 \\
\hline 3 & 81 & 0.01223 & 151 & 611.52 & 0.02743 & 2.56 & 1.84 & 7.18 \\
\hline 4 & 120 & 0.01061 & 88 & 1442.6 & 0.14201 & 9.69 & 9.37 & 3.19 \\
\hline 5 & 127 & 0.02532 & 199 & 233.07 & 0.02570 & 2.50 & 1.73 & 7.72 \\
\hline 6 & 74 & 0.01232 & 166 & 667.92 & 0.02500 & 2.89 & 1.68 & 12.1 \\
\hline 7 & 69 & 0.00925 & 134 & 1187.8 & 0.03866 & 3.75 & 2.60 & 11.5 \\
\hline 8 & 41 & 0.0043 & 105 & 2829 & 0.03251 & 2.67 & 2.19 & 4.87 \\
\hline 9 & 40 & 0.0032 & 80 & 5029.2 & 0.05501 & 3.96 & 3.69 & 2.65 \\
\hline 10 & 40 & 0.0021 & 53 & 10272 & 0.11235 & 7.86 & 7.47 & 3.94 \\
\hline 11 & 111 & 0.01611 & 145 & 535.65 & 0.04512 & 3.36 & 3.03 & 3.34 \\
\hline 12 & 119 & 0.02472 & 208 & 243.63 & 0.02359 & 2.39 & 1.59 & 8.01 \\
\hline 13 & 69 & 0.00925 & 134 & 1138.2 & 0.03705 & 3.61 & 2.49 & 11.2 \\
\hline 14 & 42 & 0.02343 & 558 & 97.455 & 0.00118 & 0.72 & 0.08 & 6.45 \\
\hline 15 & 73 & 0.02032 & 278 & 221.82 & 0.00808 & 1.29 & 0.54 & 7.46 \\
\hline 16 & 113 & 0.0159 & 141 & 563.46 & 0.04919 & 4.06 & 3.30 & 7.60 \\
\hline 17 & 88 & 0.01005 & 114 & 1203.4 & 0.06371 & 4.97 & 4.27 & 7.00 \\
\hline 18 & 131 & 0.01614 & 123 & 626.4 & 0.07349 & 7.73 & 4.92 & 28.1 \\
\hline 19 & 45 & 0.0206 & 458 & 140.56 & 0.00195 & 0.67 & 0.13 & 5.39 \\
\hline 20 & 121 & 0.02727 & 225 & 204.54 & 0.02047 & 2.46 & 1.38 & 10.9 \\
\hline 21 & 115 & 0.01577 & 137 & 598.02 & 0.05407 & 4.20 & 3.63 & 5.76 \\
\hline
\end{tabular}




\begin{tabular}{|c|c|c|c|c|c|c|c|c|}
\hline 22 & 110 & 0.01143 & 104 & 1109.20 & 0.09175 & 8.91 & 6.12 & 27.8 \\
\hline 23 & 79 & 0.0296 & 375 & 105.33 & 0.00449 & 1.15 & 0.30 & 8.43 \\
\hline 24 & 122 & 0.02514 & 206 & 248.52 & 0.02529 & 3.30 & 1.70 & 16.0 \\
\hline 25 & 75 & 0.00903 & 120 & 1171.8 & 0.04506 & 4.24 & 3.03 & 12.2 \\
\hline 26 & 69 & 0.00626 & 91 & 2257.5 & 0.07348 & 5.86 & 4.92 & 9.46 \\
\hline 27 & 40 & 0.00325 & 81 & 4915.8 & 0.05377 & 4.33 & 3.61 & 7.27 \\
\hline 28 & 131 & 0.03469 & 265 & 140.59 & 0.01649 & 3.55 & 1.11 & 24.4 \\
\hline 29 & 78 & 0.02003 & 257 & 239.46 & 0.00996 & 1.36 & 0.67 & 6.89 \\
\hline 30 & 73 & 0.01202 & 165 & 684.48 & 0.02494 & 3.02 & 1.68 & 13.2 \\
\hline 31 & 73 & 0.00894 & 122 & 1236.6 & 0.04505 & 4.31 & 3.03 & 12.9 \\
\hline 32 & 125 & 0.02633 & 211 & 226.53 & 0.02420 & 2.78 & 1.63 & 11.5 \\
\hline 33 & 130 & 0.01659 & 128 & 569.52 & 0.06529 & 5.03 & 4.38 & 6.52 \\
\hline 34 & 124 & 0.011 & 89 & 1274.6 & 0.13398 & 10.6 & 8.86 & 17.2 \\
\hline 35 & 43 & 0.02052 & 477 & 131.97 & 0.00167 & 0.59 & 0.11 & 4.74 \\
\hline 36 & 116 & 0.026 & 224 & 209.19 & 0.01924 & 2.80 & 1.29 & 15.0 \\
\hline 37 & 71 & 0.01955 & 275 & 239.52 & 0.00825 & 0.98 & 0.55 & 4.19 \\
\hline 38 & 115 & 0.01585 & 138 & 589.38 & 0.05328 & 3.90 & 3.58 & 3.21 \\
\hline 39 & 124 & 0.01599 & 129 & 619.68 & 0.06514 & 5.34 & 4.36 & 9.79 \\
\hline 40 & 124 & 0.01118 & 90 & 1212.8 & 0.12748 & 9.01 & 8.44 & 5.67 \\
\hline 41 & 118 & 0.01086 & 92 & 1372 & 0.13060 & 10.2 & 8.64 & 15.8 \\
\hline 42 & 40 & 0.0032 & 80 & 5061 & 0.05536 & 3.96 & 3.71 & 2.42 \\
\hline 43 & 40 & 0.00219 & 55 & 10272 & 0.11235 & 7.86 & 7.47 & 3.94 \\
\hline 44 & 126 & 0.02769 & 220 & 201.24 & 0.02184 & 3.46 & 1.47 & 19.9 \\
\hline 45 & 76 & 0.01132 & 149 & 777.92 & 0.03072 & 4.31 & 2.07 & 22.5 \\
\hline 46 & 71 & 0.02579 & 363 & 146.19 & 0.00504 & 1.17 & 0.33 & 8.26 \\
\hline 47 & 65 & 0.02044 & 314 & 212.16 & 0.00613 & 0.97 & 0.41 & 5.60 \\
\hline 48 & 115 & 0.02546 & 221 & 220.50 & 0.01994 & 2.21 & 1.34 & 8.66 \\
\hline 49 & 45 & 0.01619 & 360 & 223.89 & 0.00310 & 0.44 & 0.21 & 2.36 \\
\hline 50 & 65 & 0.01372 & 211 & 465.66 & 0.01345 & 1.42 & 0.90 & 5.11 \\
\hline 51 & 45 & 0.01019 & 226 & 559.44 & 0.00774 & 0.72 & 0.52 & 2.02 \\
\hline
\end{tabular}




\begin{tabular}{lcccccccc}
\hline 52 & 115 & 0.01178 & 102 & 998. & 0.09023 & 8.62 & 6.02 & 26.0 \\
53 & 65 & 0.00662 & 102 & 2172.800 & 0.06276 & 6.74 & 4.21 & 25.3 \\
54 & 45 & 0.00504 & 112 & 2254.200 & 0.03121 & 2.33 & 2.10 & 2.34 \\
55 & 40 & 0.0022 & 55 & 10979.00 & 0.12009 & 8.67 & 7.97 & 7.05 \\
56 & 122 & 0.02525 & 207 & 235.800 & 0.02399 & 2.34 & 1.61 & 7.31 \\
57 & 42 & 0.02698 & 642 & 73.952 & 0.00089 & 0.39 & 0.60 & 3.27 \\
58 & 42 & 0.03503 & 834 & 51.576 & 0.00062 & 0.35 & 0.041 & 3.12 \\
59 & 114 & 0.02649 & 232 & 207.6 & 0.01844 & 1.95 & 1.24 & 7.14 \\
60 & 41 & 0.00606 & 148 & 1278.2 & 0.01469 & 1.66 & 0.98 & 6.67 \\
61 & 40 & 0.00515 & 129 & 1893.4 & 0.02071 & 1.77 & 1.39 & 3.78 \\
62 & 113 & 0.026 & 230 & 219.69 & 0.01918 & 1.99 & 1.29 & 7.01 \\
63 & 116 & 0.02593 & 224 & 223.17 & 0.02053 & 2.07 & 1.38 & 6.89 \\
64 & 116 & 0.02581 & 223 & 228.81 & 0.02105 & 2.22 & 1.42 & 8.07 \\
65 & 115 & 0.0259 & 225 & 216.78 & 0.01960 & 2.13 & 1.32 & 8.13 \\
66 & 111 & 0.02602 & 234 & 214.02 & 0.01803 & 2.11 & 1.21 & 8.99 \\
67 & 73 & 0.00893 & 122 & 1206 & 0.04393 & 3.68 & 2.95 & 7.26 \\
\hline
\end{tabular}


Table B.2: Detailed data for measurements of damping in thin films of Al, Ag and $\mathrm{Au}$ presented in section 3 .

\begin{tabular}{|c|c|c|c|c|c|c|c|}
\hline Film & $\begin{array}{c}h_{s} \\
(\mu \mathbf{m})\end{array}$ & $\begin{array}{c}h_{f} \\
(\mathbf{n m})\end{array}$ & $\begin{array}{l}\text { Measured } 1^{\text {st }} \\
\text { mode frequency } \\
(\mathrm{Hz})\end{array}$ & $\begin{array}{c}\delta_{s} \\
\times 10^{-5}\end{array}$ & $\begin{array}{c}\delta_{C} \\
\times 10^{-5}\end{array}$ & $\begin{array}{c}\delta_{C}-\delta_{S} \\
\times 10^{-5}\end{array}$ & $\delta_{f, I F}$ \\
\hline $\mathrm{Al}$ & 117 & 61 & 114.33 & 2.11 & 5.94 & 3.83 & 0.0560 \\
\hline $\mathrm{Al}$ & 119 & 61 & 243.6 & 2.39 & 5.84 & 3.46 & 0.0514 \\
\hline $\mathrm{Al}$ & 74 & 61 & 667.8 & 2.89 & 7.29 & 4.40 & 0.0407 \\
\hline $\mathrm{Al}$ & 120 & 61 & 1442.6 & 9.69 & 13.12 & 3.44 & 0.0515 \\
\hline $\mathrm{Al}$ & 79 & 105 & 105.3 & 1.15 & 12.06 & 10.92 & 0.0626 \\
\hline $\mathrm{Al}$ & 122 & 105 & 248.49 & 3.30 & 8.53 & 5.23 & 0.0463 \\
\hline $\mathrm{Al}$ & 113 & 105 & 535.5 & 3.36 & 9.83 & 6.47 & 0.0530 \\
\hline $\mathrm{Al}$ & 75 & 105 & 1171.2 & 4.24 & 11.02 & 6.78 & 0.0369 \\
\hline $\mathrm{Al}$ & 79 & 260 & 98.565 & 1.39 & 19.12 & 17.73 & 0.0410 \\
\hline $\mathrm{Al}$ & 117 & 260 & 220.68 & 2.05 & 13.52 & 11.47 & 0.0393 \\
\hline $\mathrm{Al}$ & 81 & 260 & 611.52 & 2.56 & 16.19 & 13.63 & 0.0324 \\
\hline $\mathrm{Al}$ & 74 & 260 & 1137.8 & 3.61 & 16.71 & 13.10 & 0.0284 \\
\hline $\mathrm{Al}$ & 131 & 430 & 140.58 & 3.55 & 20.89 & 17.34 & 0.0403 \\
\hline $\mathrm{Al}$ & 78 & 430 & 239.43 & 1.36 & 30.09 & 28.73 & 0.0397 \\
\hline $\mathrm{Al}$ & 73 & 430 & 684.06 & 2.84 & 27.16 & 24.32 & 0.0315 \\
\hline $\mathrm{Al}$ & 73 & 430 & 1236 & 4.31 & 28.07 & 23.75 & 0.0307 \\
\hline $\mathrm{Ag}$ & 117 & 63 & 114.255 & 2.11 & 4.92 & 2.81 & 0.0367 \\
\hline $\mathrm{Ag}$ & 117 & 63 & 220.56 & 2.05 & 5.02 & 2.97 & 0.0387 \\
\hline $\mathrm{Ag}$ & 81 & 63 & 611.04 & 2.56 & 4.84 & 2.28 & 0.0205 \\
\hline $\mathrm{Ag}$ & 120 & 63 & 1442.6 & 9.69 & 11.20 & 1.51 & 0.0202 \\
\hline $\mathrm{Ag}$ & 79 & 110 & 98.325 & 1.39 & 7.49 & 6.10 & 0.0308 \\
\hline $\mathrm{Ag}$ & 127 & 110 & 232.74 & 2.50 & 5.92 & 3.42 & 0.0277 \\
\hline $\mathrm{Ag}$ & 74 & 110 & 666.06 & 2.89 & 7.13 & 4.24 & 0.0200 \\
\hline $\mathrm{Ag}$ & 69 & 110 & 1184 & 3.75 & 8.36 & 4.61 & 0.0203 \\
\hline
\end{tabular}




\begin{tabular}{|c|c|c|c|c|c|c|c|}
\hline $\mathrm{Ag}$ & 111 & 226 & 533.94 & 3.36 & 9.69 & 6.32 & 0.0218 \\
\hline $\mathrm{Ag}$ & 115 & 229 & 128.565 & 2.98 & 9.48 & 6.50 & 0.0229 \\
\hline $\mathrm{Ag}$ & 119 & 229 & 242.88 & 2.39 & 8.51 & 6.13 & 0.0223 \\
\hline $\mathrm{Ag}$ & 74 & 229 & 1132.4 & 3.61 & 11.48 & 7.86 & 0.0178 \\
\hline $\mathrm{Ag}$ & 42 & 443 & 96.06 & 0.72 & 33.39 & 32.67 & 0.0217 \\
\hline $\mathrm{Ag}$ & 73 & 443 & 221.12 & 1.29 & 19.05 & 17.76 & 0.0205 \\
\hline $\mathrm{Ag}$ & 113 & 443 & 561.42 & 4.06 & 14.70 & 10.64 & 0.0190 \\
\hline \multirow[t]{2}{*}{$\mathrm{Ag}$} & 88 & 443 & 1194.6 & 4.97 & 16.69 & 11.72 & 0.0163 \\
\hline & 0 & & & & & & \\
\hline $\mathrm{Au}$ & 125 & 60 & 226.08 & 2.78 & 3.73 & 0.95 & 0.0129 \\
\hline $\mathrm{Au}$ & 113 & 60 & 534.72 & 3.36 & 4.85 & 1.49 & 0.0183 \\
\hline $\mathrm{Au}$ & 130 & 60 & 568.5 & 5.03 & 5.55 & 0.52 & 0.0073 \\
\hline $\mathrm{Au}$ & 124 & 60 & 1272 & 10.57 & 10.85 & 0.27 & 0.0037 \\
\hline $\mathrm{Au}$ & 43 & 110 & 130.56 & 0.59 & 8.06 & 7.47 & 0.0190 \\
\hline $\mathrm{Au}$ & 71 & 110 & 238.11 & 1.28 & 4.22 & 2.94 & 0.0123 \\
\hline $\mathrm{Au}$ & 115 & 110 & 586.5 & 3.90 & 6.08 & 2.18 & 0.0148 \\
\hline $\mathrm{Au}$ & 118 & 110 & 1366.4 & 10.22 & 11.33 & 1.11 & 0.0077 \\
\hline $\mathrm{Au}$ & 45 & 240 & 137.955 & 0.67 & 12.97 & 12.30 & 0.0150 \\
\hline $\mathrm{Au}$ & 126 & 240 & 203.07 & 3.46 & 6.75 & 3.29 & 0.0112 \\
\hline $\mathrm{Au}$ & 124 & 240 & 614.88 & 5.01 & 8.40 & 3.39 & 0.0114 \\
\hline $\mathrm{Au}$ & 124 & 240 & 1203.2 & 9.01 & 12.05 & 3.05 & 0.0102 \\
\hline $\mathrm{Au}$ & 43 & 440 & 120.75 & 0.97 & 25.72 & 24.74 & 0.0157 \\
\hline $\mathrm{Au}$ & 121 & 440 & 205.41 & 2.47 & 9.69 & 7.23 & 0.0129 \\
\hline $\mathrm{Au}$ & 71 & 440 & 234.42 & 1.28 & 12.77 & 11.49 & 0.0121 \\
\hline $\mathrm{Au}$ & 110 & 440 & 1091.4 & 7.83 & 14.39 & 6.56 & 0.0107 \\
\hline
\end{tabular}




\section{References}

[Abd06] R Abdolvand, H Johari, G K Ho, A Erbil, F Ayazi, "Quality factor in trench-refilled polysilicon beam resonators," Journal of

Microelectromechanical Systems, vol. 15, pp. 471-478, 2006.

[Alb91] T R Albrecht, P Grutter, D Horne, D Rugar, "Frequency modulation detection using high-Q cantilevers for enhanced force microscopy sensitivity," Journal of Applied Physics, vol. 69, pp. 668 - 673, 1991

[Ban00] F D Bannon III, J R Clark, C T-C Nguyen, “ High-Q HF microelectromechanical filters," IEEE Journal of Solid-State Circuits, vol. 35, No. 4, pp. 512-526, 2000.

[Ban91] H T Banks, D J Inman, "On damping mechanisms in beams," Journal of Applied Mechanics, vol. 58, pp 716-723, 1991.

[Bau85] J Baur, A Kulik, "Optimal sample shape for internal friction measurements using a dual cantilevered beam," Journal of Applied Physics, 58 (4), 1985.

[Bee06] S P Beeby, M J Tudor, N M White, "Energy harvesting vibration sources for microsystems applications," Measurement Science and Technology, vol. 17, 12, pp 175-195, 2006.

[Ben00] J S Bendat, A G Piersol, Random Data Analysis and Measurement, Wiley-Interscience, New York, 2000.

[Ben93] J S Bendat, A G Piersol "Engineering Applications of Correlation and Spectral Analysis, second edition," Wiley-Interscience, New York, 1993.

[Ber73] C W Bert, "Material Damping: An introductory review of mathematical measures and experimental techniques" Journal of Sound and Vibration, 29, 2, pp 129-153, 1973.

[Ber75] B S Berry, W C Pritchet, "Vibrating reed internal friction apparatus for films and foils," IBM Journal of Research and Development, vol.19, pp. 334-343, 1975.

[Ber76] B S Berry, W C Pritchet, "Effect of interdiffusion on the elasticity and internal friction of compositionally modulated copper-nickel thin films," Thin Solid Films, vol. 33, pp. 19-28, 1976.

[Ber81] B S Berry, W C Pritchet, "Defect studies of thin layers by the vibratingreed technique," Journal de Physique, coll. C5, supplement 10, vol. 42, pp. 1111-1122, 1981. 
[Ber83] B S Berry, W C Pritchet, "Extended capabilities of a vibrating-reed internal friction apparatus," Review of Scientific Instruments, vol. 54, pp. 254-256, 1983.

[Ber90] B S Berry, W C Pritchet, "Internal stress and internal friction in thinlayer microelectronic materials," Journal of Applied Physics, vol. 67, pp. 3661-3668, 1990.

[Bla07] M S Blanter, I S Golovin, H Neuhauser, H R Sinning, Internal Friction in Metallic Materials, Springer, 2007.

[Boh92] H G Bohn, C M Su, "Characterization of thin films by internal friction measurements," Material Research Society Symposium Proceedings, vol. 239, pp. 215-225, 1992.

[Boil1] A Boisen, S Dohn, S S Keller, S Schmid, M Tenje "Cantilever-like micromechanical sensors," Reports of Progress in Physics, vol. 74, no. 036101, 2011.

[Bor85] C Börner, K-H Robrock, "Internal friction studies of heavy interstitial impurity atoms," Journal de Physique, col. 10, sup. 2, Tome 46, 1985.

[Bra73] W A Brantley, "Calculated elastic constants for stress problems associated with semiconductor devices," Journal of Applied Physics, vol. 44, pp. 534-535, 1973.

[Bra85] V B Braginsky, V P Mitrofanov, V I Panov, Systems with Small Dissipation, translated by E Gliner, University of Chicago Press, 1985.

[Bri00] R Brincker, L Zhang, P Andersen," Modal identification from ambient response using frequency domain decomposition," Proceedings of the 18th IMAC, S. Antonio, TX, USA 1993.

[Ca151] H A Callen, T A Welton, "Irreversibility and generalized noise," Physical Review, vol. 83, 1, pp 34 - 40, 1951.

[Cam10] H Campanella, "Acoustic Wave and Electromechanical Resonators Concept to Key Applications," Artech House, Boston, 2010.

[Cho04] D H Choi, H Kim, W D Nix, "Anelasticity and damping of thin aluminum films on silicon substrates," Journal of Microelectromechanical Systems, vol. 13, pp. 230-237, 2004.

[Cho06] D H Choi, W D Nix, "Anelastic behavior of copper thin films on silicon substrates: damping associated with dislocations," Acta Materialia, vol. 54, pp. 679-687, 2006.

[Cla05] J C Clark, M A Abdelmoneum, C T C Nguyen, "High-Q UHF micromechanical radial-contour mode disk resonators," Journal of Microelectromechanical Systems, vol. 14, 6, pp 1298-1310, 2005. 
[Duw03] A Duwel, J Gorman, M Weinstein, J Borenstein, P Ward, "Experimental study of thermoelastic damping in MEMS gyros," Sensors and Actuators A, vol. 103, pp. 70-75, 2003.

[Evo00] S Evoy, A Olkhovets, L Sekaric, J Parpia, H G Craighead, D W Carr, "Temperature-dependent internal friction in silicon nanoelectromechanical systems," Applied Physics Letters, vol. 77, pp. 2397-2399, 2000.

[Ewi00] D J Ewins, Modal Testing: Theory, Practice and Application, Research Studies Press Ltd, Hertfordshire, UK 2000.

[Fel06] M Feldman, "Time-varying vibration decomposition and analysis based on the Hilbert transform," Journal of Sound and Vibration, 295, pp 518$530,2006$.

[Fer05] A T Ferguson, L Li, V T Nagaraj, B Balachandran, B Piekarski, D L DeVoe, "Modeling and design of composite free-free beam piezoelectric resonators," Sensors and Actuators A, vol. 118, pp. 63-69, 2005.

[Fou06] B Le Foulgoc, T Bourouina, O Le Traon, A Bosseboeuf, F Marty, C Breluzeau, J P Grandchamp, S Masson, "Highly decoupled singlecrystal silicon resonators: an approach for the intrinsic quality factor," Journal of Micromechanics and Microengineering, vol. 16, pp. S45-S53, 2006.

[Fuj06] K Fujiwara, H Tanimoto, H Mizubayashi, "Elasticity study of very thin Cu films," Materials Science and Engineering A, vol. 442, pp. 336-341, 2006.

[Gab93] T B Gabrielson, "Mechanical-Thermal noise in micromachined acoustic and vibration sensors," IEEE Transactions on Electronic devices, vol. 40, 5, 1993.

[Gra75] K F Graff, Wave Motion in Elastic Solids, Dover Publications Inc, New York, 1975.

[Gra92] E J Graesser, C R Wong, "The relationship of traditional damping measures for materials with high damping capacity": a review in $M 3 D$ : Mechanics and Mechanisms of Material Damping, ASTM STP 1169, Editors: Kinra and Wolfenden, American Society for Testing and Materials, Philadelphia, pp 316-343, 1992.

[Hag02] J von der Hagen, M Weller, E Arzt, "Internal friction of copper thin layers on silicon substrates," Defect and Diffusion Forum, vols. 203-205, pp.285-288, 2002.

[Hao03] Z Hao, A Erbil, F Ayazi, "An analytical model for support loss in micromachined beam resonators with in-plane flexural vibrations," Sensors and Actuators A, vol. 109, pp. 156-164, 2003. 
[Har99] U Harms, L Kempen, H Neuhauser, "Vibrating reed apparatus with optical detection and digital processing: Applications to measurements on thin films," Review of Scientific Instruments, vol. 70, pp. 1751-1755, 1999.

[Hof77] D W Hoffman, J A Thornton, "The compressive stress transition in Al, $\mathrm{V}, \mathrm{Zr}, \mathrm{Nb}$ and $\mathrm{W}$ metal films sputtered at low working pressure," Thin Solid Films, vol. 45, pp 387-396, 1977.

[Hos95] H Hosaka, K Itao, S Kuroda, "Damping characteristics of beam-shaped micro-oscillators," Sensors and Actuators A, vol. 49, pp. 87-95, 1995.

[Hua05] X M H Huang, X L Feng, C A Zorman, M Mehregany, M L Roukes, "VHF, UHF and microwave frequency nanomechanical resonators," New Journal of Physics, 7, 247, 2005.

[Jud07] J A Judge, D M Photiadis, Vignola J F, Houston B H, Jarzynski J, "Attachment losses of micromechanical and nanomechanical resonators in the limits of thick and thin support structures," Journal of Applied Physics, 101, 013521, 2007.

[Keb90] B Kebabi, C Khan Malek, F. R Ladan, "Stress and microstructure relationships in gold thin films," Vacuum, 46, num 4-6, pp. 1353-1355, 1990.

[Kim05] B H Kim, N Stubbs, T Park, “A new method to extract modal parameters using output only responses," Journal of Sound and Vibration, 282, pp 215-230, 2005.

[Kim98] S P Kim, H M Choi, S K Choi “A study on the crystallographic orientation with residual stress and electric properties of Al films deposited by sputtering," Thin Solid Films, vol. 322, issue 1-2, pp 298$302,1998$.

[Kin94] V K Kinra, K B Milligan, "A Second - Law Analysis of Thermoelastic Damping," Journal of Applied Mechanics, vol. 61, pp. 71-76, 1994.

[Kle09] L R Klempner, R T Marinis, P Hefti, R J Pryputniewicz, "Experimental determination of the Q-factors of microcantilevers coated with thin metal films," Strain, vol. 45, pp. 295-300, 2009.

[Koc94] R Koch "The intrinsic stress of polycrystalline and epitaxial thin metal films," Journal of Physics: Condensed matter, 6 9519, 1994.

[Lan02] D Lange, O Brand, H Baltes, CMOS Cantilever Sensor Systems: Atomic Force Microscopy and Gas Sensing Applications, Springer, Berlin, 2002. 
[Lav04] N V Lavrik, M J Sepaniak, P G Datskos, "Cantilever transducers as a platform for chemical and biological sensors," Review of Scientific Instruments, vol. 75, pp. 2229-2253, 2004.

[Lee09] D Lee, S Kim, N Jing, T Thundat, S Jeon, "Effects of gold patterning on the bending profile and frequency response of a microcantilever," Journal of Applied Physics, vol. 106, art. No. 024310, 2009.

[Li07] M Li, H X Tang, M L Roukes, "Ultra-sensitive NEMS-based cantilevers for sensing, scanned probe and very high-frequency applications," Nature Nanotechnology, vol. 2, pp. 114-120, 2007.

[Lif00] R Lifshitz, M Roukes, "Thermoelastic damping in micro- and nanomechanical systems," Physical Review B, vol. 61, pp. 5600 -5609, 2000 .

[Liu99] X Liu, E Thompson, B E White, R O Pohl, "Low-temperature internal friction in metal films and in plastically deformed aluminum," Physical Review B, vol. 59, pp. 11767-11776, 1999.

[Mad02] M J Madou, Fundamentals of Microfabrication: The Science of Miniaturization, CRC Press, Boca Raton, FL, 2002.

[Mam01] H J Mamin, D Rugar, "Sub-attonewton force detection at millikelvin temperatures," Applied Physics Letters, 79, 3358, 2001.

[Mar94] N Marechal, E. Quesnel, Y Pauleau, "Silver thin films deposited by magnetron sputtering," Thin Solid Films, 241, pp 34-38, 1994.

[Mat10] D M Mattox, Handbook of Physical Vapor Deposition Processing, $2^{\text {nd }}$ edition, Elsevier Science, 2010.

[Meh07] H Mehrer, Diffusion in Solids: Fundamentals, Methods Materials, Diffusion Controlled Processes, Springer series in solid state science 155, Springer, Heidelberg, New York, 2007.

[Met09] T H Metcalf, B B Pate, D M Photiadis, B H Houston, “Thermoelastic damping in micromechanical resonators," Applied Physics Letters, vol. 95, art no. 061903, 2009.

[Mey04] E Meyer, H J Hug, R Bennewitz, Scanning Probe Microscopy: The Lab on a Tip, Springer Verlag, Berlin, 2004.

[Ngu07] C T-C Nguyen, "MEMS technology for timing and frequency control," IEEE Transactions on Ultrasonics, Ferroelectrics, and Frequency Control, vol. 54, 2, pp 251-270, 2007.

[Nis04] Y Nishino, Y Ota, T Kawazoe, "Amplitude-dependent internal friction in copper thin films on silicon substrate," Materials Science and Engineering A, vol. 370, pp. 146-149, 2004. 
[Nis06] Y Nishino, K Ikai, "Internal friction study of microplasticity in polycrystalline gold thin films," Materials Science and Engineering A, vol. 442, pp. 347-351, 2006.

[Nix88] W D Nix, "Mechanical properties of thin films," Metallurgical Transactions A, vol. 20, pp 2217-2245, 1989.

[Nou08] H Nouira, E Foltête, B Ait Brik, L Hirsinger, S Ballandras, “ Experimental characterization and modelling of microsliding on a small cantilever beam," Journal of Sound and Vibration 317, pp 30 - 49, 2008.

[Now72] A S Nowick, B S Berry, Anelastic Relaxation in Crystalline Solids, Academic Press, NY, 1972.

[Ohr02] M Ohring, Materials Science of Thin Films, $2^{\text {nd }}$ Edition, Academic Press, New York, 2002.

[Pan02] C H Pang, P Hing, A See, "Application of phase-imaging tapping-mode atomic force microscopy to investigate the grain growth and surface morphology of $\mathrm{TiSi}_{2}$," Journal of Vacuum Science and Technology, 20, Vol 6, pp 1886-9, 2002.

[Pao09] P Paolini, L Bellon, "Frequency dependence of viscous and viscoelastic dissipation in coated micro-cantilevers from noise measurements," Nanotechnology, 20, 405705, 2009.

[Pe195] V Pelosin, K F Badawi, V Branger, "Internal friction and its evolution measured on very thin platinum films," Applied Physics Letters, vol. 66, pp.691-693, 1995.

[Pho04] D M Photiadis, J A Judge, "Attachment losses of high Q oscillators," Applied Physics Letters, vol. 85, pp. 482-484, 2004.

[Ple09] M Pletea, R Koch, H Wendrock, R Kaltofen, O G Schmidt, "In situ stress evolution during and after sputter deposition of Al thin films" Journal of Physics: Condensed matter, vol. 2122500 (2009).

[Pra05] S Prabhakar, S Vengallatore, "Thermoelastic damping in bilayer composite micromechanical beam resonators," Journal of Micromechanics and Microengineering, vol. 15, pp. 2398-2404, 2005.

[Pra08] S Prabhakar, S Vengallatore, "Theory of thermoelastic damping in micromechanical resonators with two-dimensional heat conduction," Journal of Microelectromechanical Systems, vol. 17, pp. 494-502, 2008.

[Pri94] M Prieler, H G Bohn, W Schilling, H Trinkaus, "Grain boundary sliding in thin substrate-bonded Al films," Journal of Alloys and Compounds, vol. 211/212, pp. 424-427, 1994. 
[Ran02] R B Randall in Harris' Shock and Vibration Handbook, editors C M Harris, A G Piersol, McGraw Hill Handbooks, New York, 2002.

[Ra110] A A M Ralib, A N Nordin, H Salleh, "A comparative study on MEMS piezoelectric microgenerators," Microsystem Technologies, 16 (10), pp. 1673-1681, 2010.

[Rei65] F Reif, Fundamentals of Statistical and Thermal Physics, McGraw-Hill Inc, New York, 1965.

[Rei06] S Reid, G Cagnoli, D R M Crooks, J Hough, P Murray, S Rowan, M M Fejer, R Route, S Zappe, "Mechanical dissipation in silicon flexures," Physics Letters A, vol. 351, pp. 205-211, 2006.

[Ros90] T V Roszhart, "The effect of thermoelastic internal friction on the Q of micromachined Silicon resonators," in Technical Digest IEEE SolidState Sensor and Actuator Workshop, Hilton Head, SC, pp. 13-16, 1990.

[Rou66] J L Routbort, H S Sack, "Background internal friction of some pure metals at low frequencies," Journal of Applied Physics, vol. 37, pp. 4803-4805, 1966.

[San05] R Sandberg, K Molhave, A Boisen, W Svendsen, "Effect of gold coating on the Q-factor of a resonant cantilever," Journal of Micromechanics and Microengineering, vol. 15, pp. 2249-2253, 2005.

[Sch83] R B Schwarz, L L Funk, "Internal friction study of solute segregation to dislocations" Acta Metallica, vol. 31, 2, pp 219-315, 1983.

[Sek02] L Sekaric, D W Carr, S Evoy, J M Parpia, H G Craighead, "Nanomechanical resonant structures in silicon nitride: fabrication, operation and dissipation issues," Sensors and Actuators A, vol. 101, pp. 215-219, 2002.

[Sha11] N Shalabi, "Design, assembly and validation of a system to measure damping,” M. Eng Thesis, McGill University, Montreal, 2011.

[Sil00] C W De Silva, Vibration fundamentals and practice, Boca Raton, CRC press LLC, 2000.

[Smi95] D Smith, "Limits of force microscopy," Review of Scientific Instruments, vol. 66, 5, pp 3191-95, 1995.

[Smi96] C B Smith, N M Wereley, "Transient analysis for damping identification in rotating composite beams with integral damping layers" Smart Materials and Structures, vol. 5, pp. 540-550, 1996. 
[Sos10] G Sosale, S Prabhakar, L Fréchette, S Vengallatore, "Effect of thin aluminum coatings on structural damping of silicon microresonators," Material Research Society Symposium Proceedings, vol. 1222, pp. 8388, 2010.

[Sp107] M Spletzer, A Raman, "Elastomeric sensing using higher flexural eigenmodes of microcantilevers," Applied Physics Letters, vol. 91, no. $184103,2007$.

[Sri02] V T Srikar, S D Senturia, "Thermoelastic damping in fine-grained polysilicon flexural beam resonators," Journal of

Microelectromechanical Systems, vol. 11, pp. 499-504, 2002.

[Sto09] G G Stoney, "The tension of metallic films deposited by electrolysis" Proceedings of the Royal Society, London, A, 82, pp. 172, 1909.

[Su94] C M Su, M Wuttig, "Internal friction in thin films and membranes," Journal of Alloys and Compounds, vol. 211/212, pp. 428-433, 1994.

[Sur03] S Suresh, L B Freund, Thin Film Materials, Cambridge University Press, 2003.

[Tam06] J Tamayo, D Ramos, J Mertens, M Calleja, "Effect of the adsorbate stiffness on the resonance response of microcantilever sensors," Applied Physics Letters, 89, 224104, 2006.

[Tay82] J R Taylor, An Introduction to Error Analysis The Study of Uncertainties in Physical Measurements, $2^{\text {nd }}$ Edition, University Science Books, Sausalito, California, 1982.

[Tho78] W Thomson (Lord Kelvin), "On the Thermoelastic, Thermomagnetic and Pyro-electric Properties of Matters", Philosophical Magazine, vol. 5, pp. 4-27, 1878.

[Tho77] J A Thornton, "High rate thick film growth," Annual review of material science, 7, pp. 239-260, 1977.

[Tho89] J A Thornton, D W Hoffeman, "Stress related effects in thin films," Thin solid films 171, pp 5-31, 1989.

[Ton99] Q Y Tong, U Gösele, Semiconductor Wafer Bonding: Science and Technology, Wiley-Interscience, New Jersey, 1999.

[Ven05] S Vengallatore, "Analysis of thermoelastic damping in laminated composite micromechanical beam resonators," Journal of Micromechanics and Microengineering, vol. 15, pp. 2398-2404, 2005. 
[Ven08] S Vengallatore. "Structural damping in micro/nanomechanical resonators: Mechanisms, models and design implications," in Proceedings of ISSS 2008: International Conference on Smart Materials Structures and Systems, Bangalore, India 2008.

[Wag09] P S Waggoner, H G Craighead, "The relationship between material properties, device design and the sensitivity of resonant mechanical sensors," Journal of Applied Physics, 105, 054306, 2009.

[Wea90] W Weaver JR, S P Timoshenko, D H Young, Vibration Problems in Engineering, $5^{\text {th }}$ edition, Wiley-Interscience, New York, 1990.

[Wei63] G P Weiss, D O Smith, "Measurement of internal friction in thin films," Review of Scientific Instruments, vol. 34, pp.522-523, 1963.

[Win92] H Windischmann, "Intrinsic stress in sputter deposited thin films," Critical reviews in solid state and materials science, 17(6), pp 547-996, 1992.

[Yan00] J Yang, T Ono, M Esashi, "Surface effects and high quality factors in ultrathin single-crystal silicon cantilevers," Applied Physics Letters, vol. 77, pp. 3860-3862, 2000.

[Yan06] Y T Yange, C Callegari, X L Feng, K L Ekinci, M L Roukes, "Zeptogram-scale nanomechanical mass sensing," Nanoletters, 6, 4, pp 583-586, 2006.

[Yan96] S Yang, R F Gibson, G M Crosbie, R L Allor, "Measurement of dynamic mechanical properties of advanced ceramics and ceramic matrix composites at temperatures above $1000^{\circ} \mathrm{C}$," Experimental Techniques, vol. 20, 3, pp 20-23, 1996.

[Yas00] K Y Yasumura, T D Stowe, E M Chow, T Pfafman, T W Kenny, B C Stipe, D Rugar, "Quality factors in micro- and submicron-thick cantilevers," Journal of Microelectromechanical Systems, vol. 9, pp. 117-125, 2000.

[Zen37] C Zener, "Internal friction in solids I: Theory of internal friction in reeds," Physical Review, vol. 52, pp. 230-235, 1937.

[Zen38] C Zener, "Internal friction in solids II: General theory of thermoelastic internal friction," Physical Review, vol. 53, pp. 90-99, 1938.

[Zen47] C Zener, "Mechanical behavior of high damping materials," Journal of Applied Physics, vol. 18, iss 11, pp 1022-25, 1947.

[Zhu95] A W Zhu, H G Bohn, W Schilling, "Internal friction associated with grain boundary diffusion in gold films," Philosophical Magazine A, vol. 72, pp. 805-812, 1995. 